\title{
Charakterisierung elektronischer und magnetischer Eigenschaften in Seltenen Erd-Borkarbiden
}

\author{
Dissertation \\ zur Erlangung des Doktorgrades \\ der Mathematisch-Naturwissenschaftlichen Fakultäten \\ der Georg-August-Universität zu Göttingen
}

vorgelegt von

Klaus Krug

aus

Kassel

Göttingen 2000 
Referent:

Korreferent:

Tag der mündlichen Prüfung: 21. Juni 2000
Prof. Dr. K. Winzer

Prof. Dr. H. C. Freyhardt 


\section{Inhaltsverzeichnis}

1 Einleitung....................................................................................................................................1

2 Experimentelles ......................................................................................................................................8

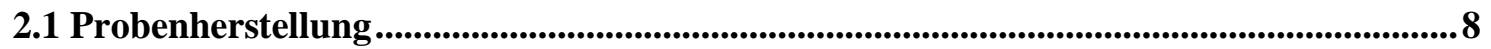

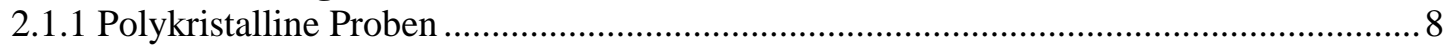

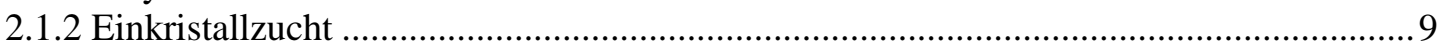

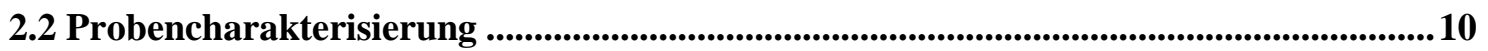

2.2 .1 Widerstandsmessungen .....................................................................................

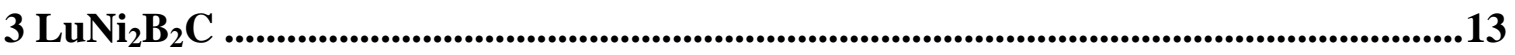

3.1 Vorarbeiten aus der Literatur ..........................................................................................13

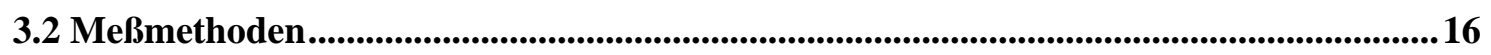

3.3 Der de Haas-van Alphen-Effekt ......................................................................................................19

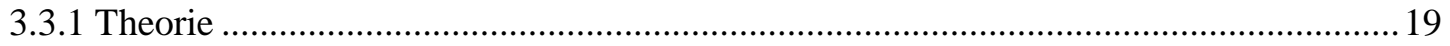

3.3.2 Der dHvA-Effekt im supraleitenden Zustand ........................................................ 27

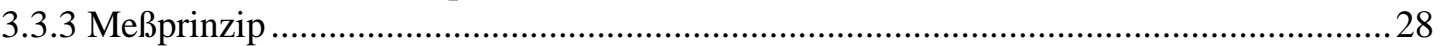

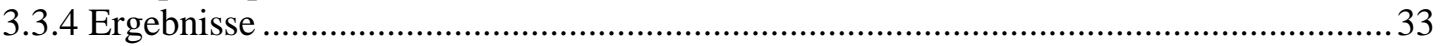

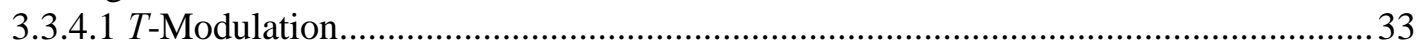

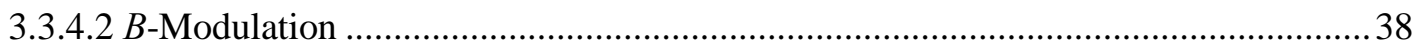

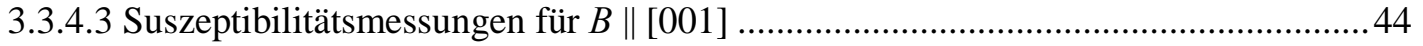

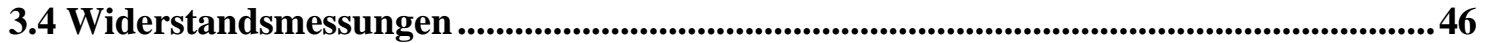

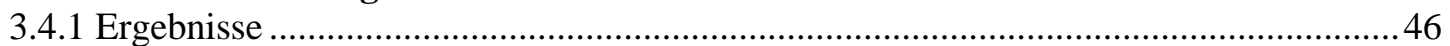

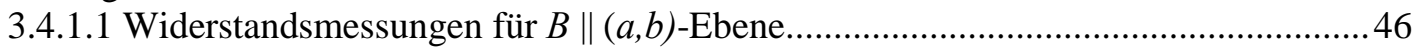

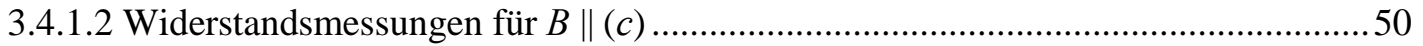

3.5 Diskussion .....................................................................................................................................................52

$4 \mathrm{DyNi}_{2} \mathrm{~B}_{2} \mathrm{C}$.............................................................................................................................63

4.1 Vorarbeiten aus der Literatur .........................................................................................................63

4.2 Meßmethoden.........................................................................................................................69

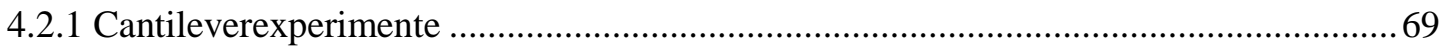

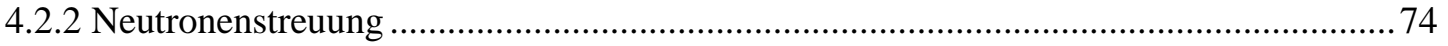

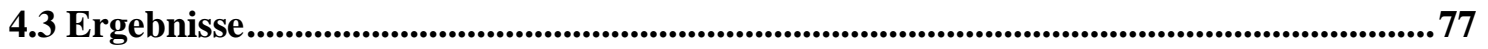

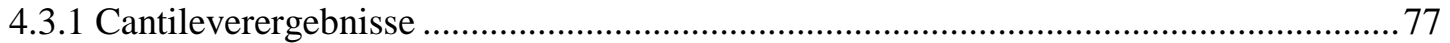

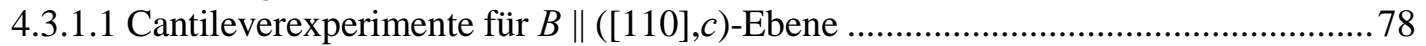

4.3.1.2 Cantileverexperimente für $B \|([100], c)$-Ebene ................................................ 79

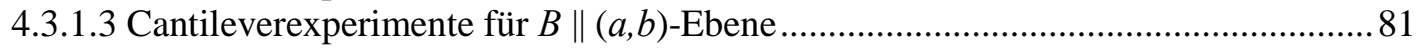

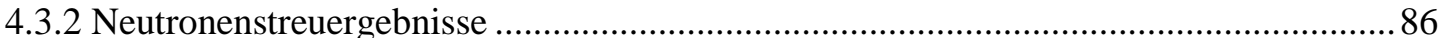

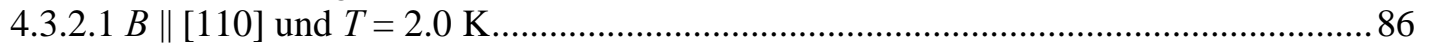

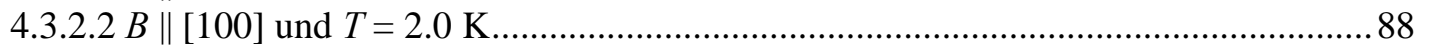

4.4 Diskussion ..............................................................................................................................................90

5 Zusammenfassung...............................................................................................92

6 Literaturverzeichnis................................................................................................94 


\section{Einleitung}

Das gleichzeitige Auftreten von Supraleitung und Magnetismus in einer Probe: Anreiz und physikalische Herausforderung für Festkörperphysiker seit den ersten Arbeiten auf diesem Gebiet Ende der 50er Jahre [Mat58]. Die vorliegende Arbeit folgt dem seit Matthias et al. kontinuierlich fortgeschrittenem Weg zur Erforschung dieses Phänomens durch Charakterisierung der elektronischen und magnetischen Eigenschaften der 1994 entdeckten Klasse der Borkarbide, welche Koexistenz von Supraleitung und magnetischer Ordnung zeigen [Nag94].

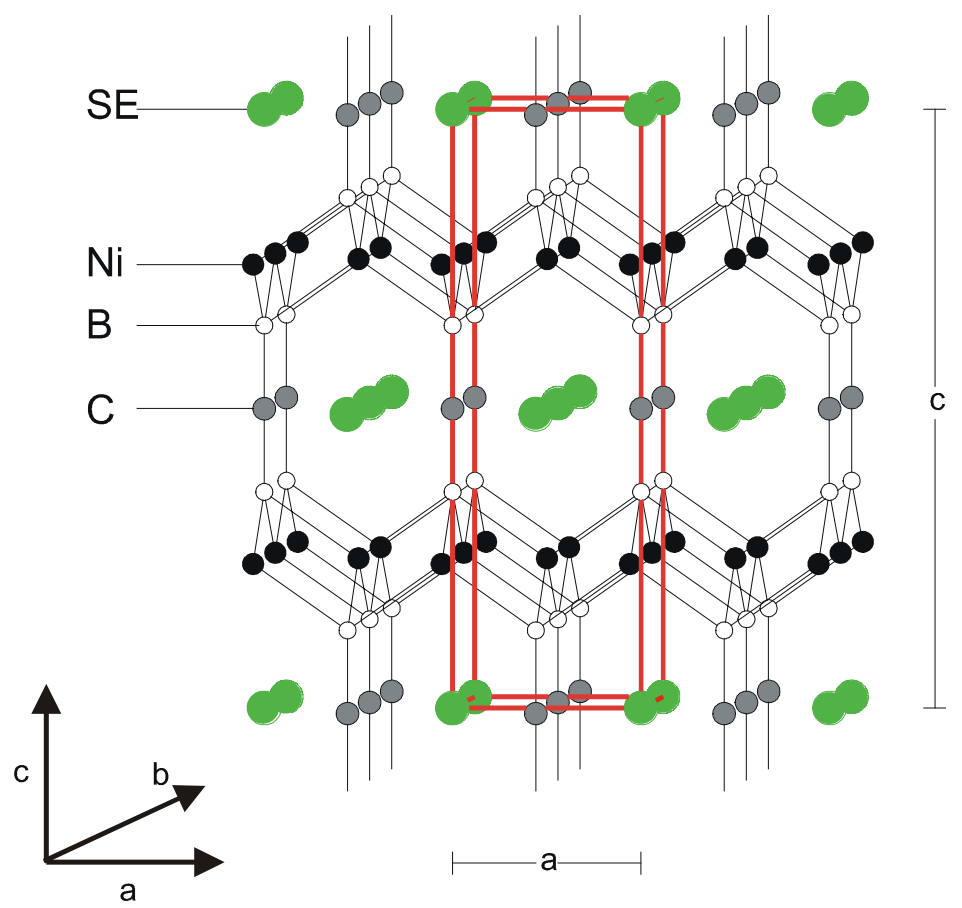

Abb.1.1 Tetragonal- raumzentrierte Kristallstruktur der $S E(\ddot{U} M)_{2} B_{2} C$ mit $\ddot{U} M \cong N i$ nach Siegrist et al. [Sie94]. Obwohl die schichtartige Struktur aus alternierenden $\mathrm{Ni}_{2}-B_{2}$ und $S E-C$ Ebenen an die Hochtemperatursupraleiter erinnert, zeigen die Borkabide 3-dimensionale Supraleitung.

Obwohl die 1986 entdeckten oxidischen Supraleiter [Bed86], [Bed87] mittlerweile auch auf diesem Forschungsgebiet an Aufmerksamkeit gewinnen [Pin99], so formten schon früheste Experimente ein einprägsames Bild für die allgemein beobachtete Störung der Supraleitung durch probeninhärenten Magnetismus: Legierungen des unmagnetischen und unterhalb von Sprungtemperaturen $T_{\mathrm{c}} \leq 5.9 \mathrm{~K}$ supraleitenden Lanthans mit paramagnetischen Seltenen Erd (SE) - Zusätzen von einem Atomprozent erschweren die Bildung 
von supraleitenden Cooperpaaren aus „Spin up“- und „Spin down“- Leitungselektronen. Bereits ein Atomprozent magnetischer Verunreinigungen führt durch die Spin-Streuung der Leitungselektronen mit dem Spin der magnetischen Verunreinigung zu signifikanten Absenkungen der Sprungtemperatur $T_{\mathrm{c}}$, je nach Größe des Spins der eingebrachten Seltenen Erde [Mat58]. Im Grenzfall kleiner Konzentrationen beobachtet man eine lineare Absenkung in $T_{\mathrm{c}}$, was quantitativ durch die Abrikosov-Gorkov-Theorie [Abr61] beschrieben wird.

Mit diesem einfachen Bild für die Paarbrechung von Cooperpaaren durch magnetisch ungeordnete Verunreinigungen schien die Existenz eines Systems unmöglich, das gleichzeitig sowohl Supraleitung als auch makroskopische magnetische Ordnung zeigt. Zur Einstellung einer magnetischen Ordnung bedarf es einer Wechselwirkung (WW) der Momente. Dies ist am einfachsten durch eine Erhöhung des atomaren Anteils und damit geringeren Abständen der magnetischen Momente in der Verbindung zu realisieren. Mit der geschilderten Konzentrationsabhängigkeit von $T_{\mathrm{c}}$ sollte dies aber das Auftreten von Supraleitung gänzlich unterdrücken.

Auf besonderes Interesse stießen daher in den späten 70er Jahren die nach ihrem Entdekker benannte „Chevrel-Phase“ $S E \mathrm{Mo}_{6} \mathrm{~S}_{8}$ bzw. $S E \mathrm{Mo}_{6} \mathrm{Se}_{8}$ und die Seltenen Erd Rhodium Boride $\mathrm{SERh}_{4} \mathrm{~B}_{4}$ (eine Übersicht findet sich in [Sin89] oder [Mat84]). Bei diesen koexistiert magnetische Ordnung der $S E$-Momente mit der Supraleitung unterhalb von $T_{\mathrm{c}} \leq 12 \mathrm{~K}$ und verblüffende Hysterese- und ,reentrant"-Effekte in den supraleitenden Eigenschaften wurden bspw. an $\mathrm{ErRh}_{4} \mathrm{~B}_{4}$ beobachtet.

Anfang der 90er Jahre gelang die Entdeckung der im Rahmen dieser Arbeit untersuchten Borkarbidverbindungen. Nagarajan et al. berichteten 1994 [Nag94] als erste von Supraleitung im Borkarbid $\mathrm{YNi}_{4} \mathrm{BC}_{\delta}$ unterhalb $T_{\mathrm{c}} \approx 12 \mathrm{~K}$. Nahezu zeitgleich bestimmte die Arbeitsgruppe um R. Cava und T. Siegrist [Sie94] die genaue Stöchiometrie der Phase mit höchstem $T_{\mathrm{c}}$ in einer ganzen Klasse von $S E$-Übergangsmetall( $\left.\ddot{U} M\right)$-Borkarbiden, nämlich $S E(\ddot{U} M)_{2} \mathrm{~B}_{2} \mathrm{C}$, mit ihrer zugehörigen tetragonalen Kristallstruktur (siehe Abb.1.1). Die mögliche Substitution des Übergangsmetalls (u.a. [Sch94], [Cav94], [Bud95]) brachte mit $\mathrm{YPd}_{2} \mathrm{~B}_{2} \mathrm{C}$ bereits frühzeitig [Cav94] die Verbindung mit dem bis heute höchsten $T_{\mathrm{c}} \approx 23 \mathrm{~K}$ in der Klasse hervor. Den eigentlichen Reiz für die Erforschung 
der WW von Supraleitung und Magnetismus stellt allerdings die $S E$-Substitution dar. Die $S E$ liegen in Verbindungen zumeist als dreiwertig positive $S E^{3+}$ vor, genauso wie Y, La, und Sc. Dank dieser chemischen Ähnlichkeit der SE, denen im folgenden auch die Elemente Sc und Y zugeordnet werden, gelang es alsbald fast die ganze Serie von $S E$ $\mathrm{Ni}_{2} \mathrm{~B}_{2} \mathrm{C}$-Borkarbiden stabil herzustellen. Durch das mit der $4 \mathrm{f}-$ Elektronenzahl variierende magnetische Moment der $S E$ wurden dabei frühzeitig Variationen der magnetischen und supraleitenden Eigenschaften beobachtet [Can97a], [Mas95].

Zusätzlich zur reinen Dipol-Dipol-WW tritt in den Borkarbiden die von den Leitungselektronen übertragene indirekte Ruderman-Kittel-Kasuya-Yosida (RKKY)-WW [Zim75] zwischen den $S E$-Momenten auf, welche Néeltemperaturen $T_{\mathrm{N}}$ unterhalb $20 \mathrm{~K}$ ermöglicht [Lyn97]. Da die schweren $S E$-Borkarbide wie Dy-, Ho-, Er- und $\mathrm{TmNi}_{2} \mathrm{~B}_{2} \mathrm{C}$ Koexistenz von Supraleitung $\left(T_{\mathrm{c}} \leq 10.9 \mathrm{~K}\right)$ und Antiferromagnetismus $\left(T_{\mathrm{N}} \leq 10.3 \mathrm{~K}\right)$ in einem leicht zugänglichen Temperaturbereich zeigen, sind die Borkarbide heute das Paradesystem zur Erforschung der WW dieser beiden Ordnungseffekte. Als Funktion der SE sind Variationen des Verhältnisses $r \equiv T_{\mathrm{N}} / T_{\mathrm{c}}$ von $\mathrm{r}=0(\mathrm{Y}, \mathrm{Lu})$ über $r \approx 1(\mathrm{Ho}, \mathrm{Dy})$ bis $r>1(\mathrm{~Tb})$ möglich, welche als Maß für das Energieverhältnis von Magnetismus zu Supraleitung dienen können.

Übergreifend zeigen die supraleitenden Borkarbide eine Fülle von interessanten Phänomenen, die zuvor einzeln zu Forschungsschwerpunkten der Supraleitungs - oder Festkörperforschung zählten und in ersten Übersichtsartikeln komprimiert dargestellt werden [Can98], [Hil99], [Dre99]. Als Beispiele sollen hier die Beobachtung von quadratischen Flußliniengittern und Flußliniengitterübergängen [Esk97], [Wi197], de Haas-van AlphenOszillationen im supraleitenden Zustand [Hei95], Fermiflächen Nesting [Kim95], mögliche $d$-Wellen-Supraleitung [Met97], [Wan98], ,reentrant“ Supraleitung [Kru96], [Pen98] oder das Ausbilden von feldstabilisiertem Metamagnetismus [Can97a], [Mas95] genannt werden.

Das Verständnis von Systemen mit $r \approx 1$, bei denen der Wettstreit zwischen Supraleitung und Magnetismus wegen nahezu gleicher Ordnungsenergien am ausgeprägtesten sein sollte, muß durch eine möglichst detaillierte Charakterisierung der unmagnetischen Sy- 
steme wie $\mathrm{YNi}_{2} \mathrm{~B}_{2} \mathrm{C}$ und $\mathrm{LuNi}_{2} \mathrm{~B}_{2} \mathrm{C}$ erfolgen, in Verbindung mit einer Überprüfung der Übertragbarkeit der dort gefundenen Erkenntnisse auf die magnetischen Systeme.

Für die unmagnetischen Borkarbide gab es durch die Beschreibung der elektronischen Eigenschaften mittels Bandstrukturrechnungen [Mat94], [Pic94] einen anfänglichen Vorsprung der theoretischen Arbeiten, der mittlerweile durch eine Vielzahl von durchgeführten Experimen aufgeholt wurde. Demnach ist die im Experiment beobachtete 3dimensionale Supraleitung [Rat97] im Einklang mit den berechneten 3-dimensionalen Fermiflächen [Pic94], [Mat94a], [Dre99]. Außerdem schien die berechnete Abnahme der elektronischen Zustandsdichte an der Fermikante $N\left(E_{\mathrm{F}}\right)$ beim Übergang von $\mathrm{LuNi}_{2} \mathrm{~B}_{2} \mathrm{C}$ zu LuNiBC die experimentell beobachtete $T_{\mathrm{c}}$-Abnahme von $16.3 \mathrm{~K}\left(\mathrm{LuNi}_{2} \mathrm{~B}_{2} \mathrm{C}\right)$ auf $2.9 \mathrm{~K}$ (LuNiBC) im Bild von konventioneller BCS-Supraleitung nach Bardeen-CooperSchrieffer [Bar57] zu erklären. Mit der Zahl der experimentellen Daten mehren sich aber die Phänomene, welche nicht zweifelsfrei mit der isotropen $B C S$-Theorie vereinbar sind. So kann die erwartete Skalierung von $T_{\mathrm{c}}$ mit $N\left(E_{\mathrm{F}}\right)$ nicht mit den heute zur Verfügung stehenden experimentellen Daten in Einklang gebracht werden, wenn man außer den Beispielen $\mathrm{LuNi}_{2} \mathrm{~B}_{2} \mathrm{C}$ und $\mathrm{LuNiBC}$ noch weitere $S E$-Übergangsmetall( $\left.\ddot{U} M\right)$-Borkarbide betrachtet [Hil99], [Dre99]. Vielmehr deutet eine Reihe von Experimenten ([Noh97], [Met97], [Mun96], [Hei95]) auf unkonventionelle $d$-Wellen- [Mak96] oder anisotrope $s$ Wellen-Supraleitung im Eliashberg-Formalismus [Eli60] hin. So würde das in diesen Beschreibungen mögliche partielle Verschwinden der Energielücke $\Delta$ die teilweise ungedämpfte Beobachtung von de Haas-van Alphen-Oszillationen bei Eintritt der Supraleitung im Y-System oder die beobachtete $\gamma \propto \sqrt{B}$-Magnetfeldabhängigkeit der Sommerfeldkonstanten im Lu-System erklären. Desweiteren deuten Wang et al. [Wan98] die in $\mathrm{LuNi}_{2} \mathrm{~B}_{2} \mathrm{C}$ innerhalb der $(a, b)$-Ebene beobachtete $B_{\mathrm{c} 2}$-Anisotropie mittels $d$-WellenSupraleitung.

Um weiteren Aufschluß über die elektronische Struktur der Borkarbide und Indizien für den zur Supraleitung führenden Mechanismus zu erhalten, wurden in dieser Arbeit ergänzende Experimente an qualitativ hochwertigen $\mathrm{LuNi}_{2} \mathrm{~B}_{2} \mathrm{C}$-Einkristallen durchgeführt. Die Präparation der Einkristalle erfolgte mit einem Flußmittel-Zuchtverfahren nach Cho et al. [Cho95a], das in Kapitel 2.1.2 vorgestellt wird. In Kapitel 3 folgt, nach einer Einführung 
der aus Vorarbeiten bekannten Daten zum Lu-System, die Präsentation und Diskussion der aus de Haas-van Alphen-Experimenten und aus anisotropen Magnetowiderstandsmessungen ermittelten Ergebnisse.

Die Charakterisierung der anisotropen magnetischen Eigenschaften erfolgte für beinahe alle Borkarbide sowohl durch makroskopische Meßmethoden wie spezifische Wärme ([Cho95a], [Can96], [Hi199]), Magnetowiderstand ([Pen98], [Rat96]) oder Magnetisierungsmessungen ([Can97a], [Nau98]) als auch durch mikroskopische wie die Neutronenstreuung an $\mathrm{SENi}_{2}\left({ }^{11} \mathrm{~B}\right)_{2} \mathrm{C}([\mathrm{Gol} 94]$, [Lyn97]) im Nullfeld. Die makroskopischen Verfahren ergaben detaillierte magnetische Phasendiagramme in der $B / T$-Ebene [Can97a] mit einer Vielzahl von metamagnetischen Zwischenphasen beim Übergang vom Antiferromagnetismus $(A F M)$ in durch äußere Magnetfelder gesättigte Zustände. Mikroskopisch ergaben Neutronenstreuexperimente diskrete Einstellungen der magnetischen Momente sowohl in den zumeist kommensurablen als auch den inkommensurablen antiferromagnetischen $(\mathrm{afm})$ Grundzuständen. Die diskreten Spinstellungen in Verbindung mit leichten Richtungen der Magnetisierung sind durch Kristallfeldaufspaltungen am $S E$ - Platz begründet, welche die über die RKKY-WW ordnenden Momente auf diese Richtungen beschränken [Cho96] [Lyn97]. Die Interpretation dieser magnetischen Phasen und ihre Auswirkungen auf die Supraleitung sind für die Systeme $\mathrm{HoNi}_{2} \mathrm{~B}_{2} \mathrm{C}, \mathrm{ErNi}_{2} \mathrm{~B}_{2} \mathrm{C}$ und $\mathrm{DyNi}_{2} \mathrm{~B}_{2} \mathrm{C}$ mit $r_{\mathrm{Ho}} \approx 5 / 8, \mathrm{r}_{\mathrm{Er}} \approx 7 / 11$ und $r_{\mathrm{Dy}} \approx 10 / 6$ wohl am spannendsten.

Die meisten Arbeiten hierzu erfolgten bislang zum Ho-System (u.a. [Rat96], [Kru96], [Ami98], [Kre99]). Ein dort beobachteter ,reentrant“-Effekt zur Normalleitung durch Einsetzen von magnetischer Ordnung im supraleitenden Zustand wurde frühzeitig im Rahmen der Abrikosov-Gorkov-Theorie beschrieben [Kru96]. Die Vorstellung von unabhängig wirkenden Paarbrechungsanteilen durch die aus Neutronenstreuung im Nullfeld bekannten verschiedenen magnetischen Phasen wurde mittlerweile bestätigt und um die detaillierten Auswirkungen der felderzeugten Phasen auf die Supraleitung mittels eines mikroskopischen Modells erweitert [Ami00]. Inspirierend für die Beschreibung der felderzeugten metamagnetischen Phasen in den Borkarbiden ist die experimentell und theoretisch begründete Annahme eines sogenannten ,4-clock“-Modells der magnetischen Momente [Can97], [Kal98], [Ami98]. 


\section{$A F M$}

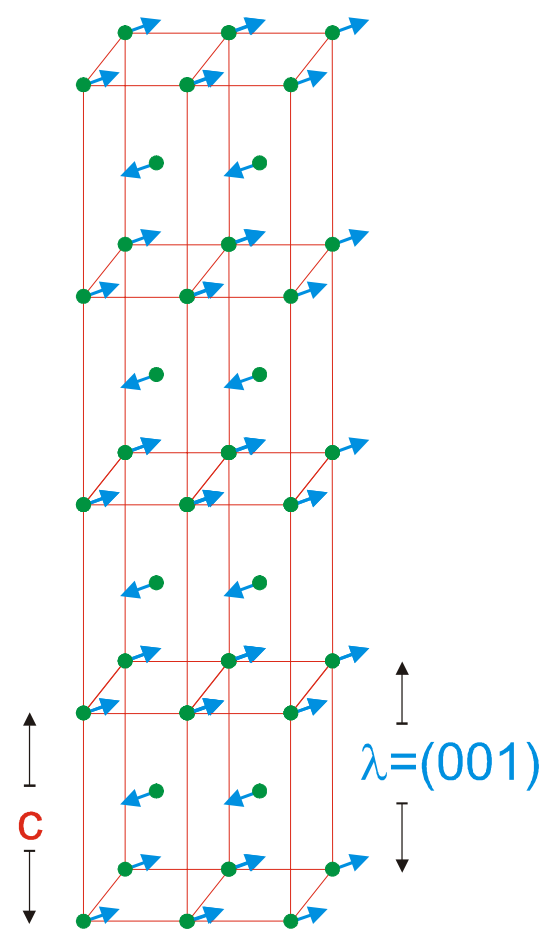

M2

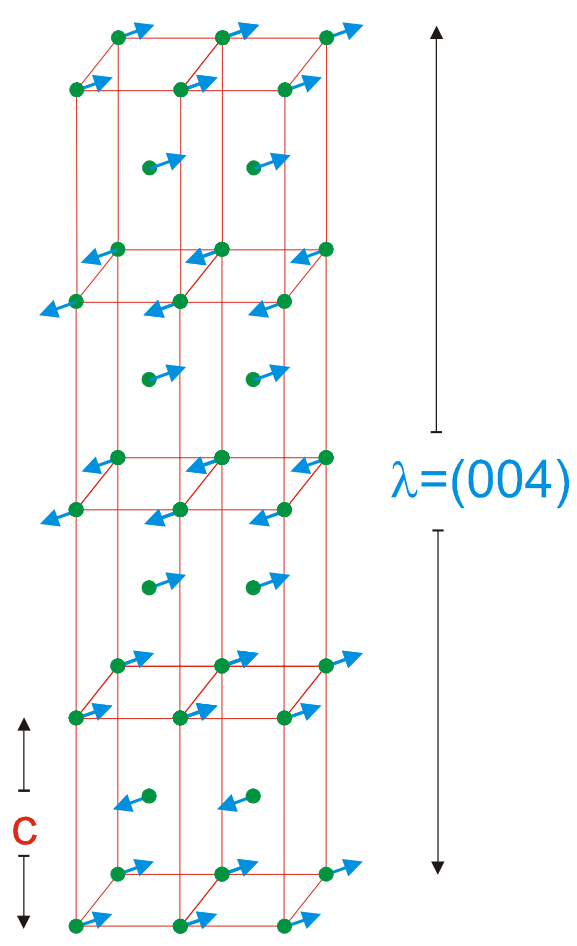

Abb.1.2 Schematische Darstellung zweier magnetischer Strukturen im „,4-clock“-Model. Links die afm Struktur, wie sie in $\mathrm{DyNi}_{2} \mathrm{~B}_{2} \mathrm{C}$ und $\mathrm{HoNi}_{2} \mathrm{~B}_{2} \mathrm{C}$ beobachtet wird. Rechts eine mögliche Realisierung des in DyNi ${ }_{2} B_{2} C$ auftretenden metamagnetischen Zustandes $M 2$ mit $M_{\text {eff }}=1 / 4 \cdot M_{F M}$. $\lambda$ bezeichnet jeweils die Periodizitätslänge der magnetischen Strukturen im Ortsraum.

Es basiert auf der durch das Kristallfeld erzeugten Einschränkung der magnetischen Momente in leichte Richtungen der Magnetisierung, welche speziell für das Ho- und DySystem entlang der vier $<110>$-Richtungen weisen. Die felderzeugten metamagnetischen Phasen lassen sich in diesem Modell als in den leichten Richtungen des Systems ferromagnetisch $(f m)$ ausgerichtete $(a, b)$-Ebenen der magnetischen Momente, mit unterschiedlicher Abfolge der Stapelung entlang der $c$-Richtung identifizieren (siehe Abb.1.2). Die so konstruierten metamagnetischen Phasen sind nicht nur in Einklang mit den experimentellen Magnetisierungswerten, sondern auch mit den dort gefundenen Winkelabhängigkeiten für die Übergangsfelder zwischen den Phasen [Can97]. Diese für das Ho-System vorgeschlagene Identifizierung der metamagnetischen Phasen wurde basierend auf orientierungsabhängigen Magnetisierungs- und Widerstandsmessungen ([Can97a], [Pen98]) von Winzer et al. [Win99b] auf das Dy-Borkarbid konsistent übertragen, wobei noch 
Spielraum für die genaue Form der Stapelfolgen besteht. Die mikroskopische Überprüfung dieses Modells stand bislang aber noch aus. Daher dreht sich der im zweiten Teil dieser Arbeit gelegte Forschungsschwerpunkt um die erweiterte Charakterisierung der metamagnetischen Phasen im $\mathrm{DyNi}_{2} \mathrm{~B}_{2} \mathrm{C}$-System. In Kap 4 werden die eigenen experimentellen Ergebnisse in Verbindung mit den Vorarbeiten zum Dy-System präsentiert und diskutiert.

Als experimentelle Methode kam dabei ein neu in Betrieb genommenes Cantilevermagnetometer zum Einsatz, welches im sogenannten Drehmomentmodus $\vec{\tau}=\vec{M} \times \vec{B}$ mit Empfindlichkeiten der Größenordnung $10^{-5} \mathrm{Nm} / \mathrm{T}$ äußerst sensitiv auf anisotrope Probenmagnetisierungen anspricht. Dies und die erstmalig an $\mathrm{DyNi}_{2} \mathrm{~B}_{2} \mathrm{C}$-Einkristallen durchgeführte Neutronenstreuung an Einkristallen (Kap.4.2) diente zur Überprüfung der aus makroskopischen Methoden gewonnenen Beschreibung des Metamagnetismus im „4clock“-Modell.

Diese Arbeit schließt in Kap.5 mit der Zusammenfassung der an Lu- und $\mathrm{DyNi}_{2} \mathrm{~B}_{2} \mathrm{C}$ gewonnenen Ergebnisse. 


\section{Experimentelles}

\subsection{Probenherstellung}

Verbindungen der Form $S E \mathrm{Ni}_{2} \mathrm{~B}_{2} \mathrm{C}$ mit verschieden Elementen aus der Gruppe der Seltenen Erden $(S E)$ zuzüglich Lutetium und Yttrium wurden in unserer Arbeitsgruppe bereits kurz nach der Entdeckung von Supraleitung in $\mathrm{LuNi}_{2} \mathrm{~B}_{2} \mathrm{C}$ [Nag94] erfolgreich hergestellt [Hei95]. Die Vorgehensweise ist an die von Cho et al. [Cho95a] veröffentlichte angelehnt. Für Neutronenstreuexperimente ist das elementare B durch isotopenreines elementares ${ }^{11} \mathrm{~B}$ zu ersetzen, da dies einen kleineren Absorptionsquerschnitt für Neutronen aufweist. Für die Herstellung von $\operatorname{DyNi}_{2}\left({ }^{11} \mathrm{~B}\right)_{2} \mathrm{C}$-Einkristallen wurde das isotopenreine ${ }^{11} \mathrm{~B}$ sowohl für den polykristallinen $\mathrm{DyNi}_{2}\left({ }^{11} \mathrm{~B}\right)_{2} \mathrm{C}$-Schmelzling als auch das Flußmittel $\mathrm{Ni}_{2}\left({ }^{11} \mathrm{~B}\right)$ verwendet.

\subsubsection{Polykristalline Proben}

Die in ihrem stöchiometrischen Verhältnis eingewogenen Ausgangsmaterialien der gewünschten $S E \mathrm{Ni}_{2} \mathrm{~B}_{2} \mathrm{C}$-Verbindung werden im Lichtbogenofen unter Argonatmosphäre Element für Element miteinander verschmolzen. Das schrittweise Verschmelzen ermöglicht es, bezüglich der Einwaage auftretende Masseverluste, etwa durch „Wegspritzen“ von Probenmaterial im Lichtbogen, zu kontrollieren und gegebenenfalls zu korrigieren.

Man beginnt mit den beiden Elementen mit niedrigsten Schmelzpunkten $S E\left(T_{\mathrm{M}} \approx 1400^{\circ} \mathrm{C}\right.$; Reinheit $\left.3 \mathrm{~N}\right)$ und $\mathrm{Ni}\left(1455^{\circ} \mathrm{C} ; 4 \mathrm{~N}\right)$, bei denen nahezu kein Masseverlust beim Verschmelzen auftritt. Beim Verschmelzen von Bor $\left(2092^{\circ} \mathrm{C} ; 5 \mathrm{~N}\right)$ mit der Verbindung $S E-\mathrm{Ni}_{2}$ kommt es häufig zum Zerreißen und Wegspritzen von Bor-Partikeln, verursacht durch innere Spannungen im schlecht wärmeleitenden Bor durch unvermeidbare Temperaturgradienten. Nach zwei- bis dreimaligem Erhitzen im Lichtbogen und anschließender Nachwaage gelingt es auch in diesem Schritt die erforderliche Stoffmenge an $S E-\mathrm{Ni}_{2}-\mathrm{B}_{2} \mathrm{zu}$ verschmelzen.

Als letztes Element verbindet man den Kohlenstoff $\left(3375^{\circ} \mathrm{C} ; 5 \mathrm{~N}\right)$ mit $\mathrm{SE}-\mathrm{Ni}_{2}-\mathrm{B}_{2}$. Hier ist das Manko des Wegsprühens dadurch vermindert, daß das pulverförmige Ausgangsmaterial in Plättchen gepreßt wurde, die in der richtigen Stöchiometrie von der $S E-\mathrm{Ni}_{2}-\mathrm{B}_{2-}$ 
Schmelze aufgenommen werden. Zum Lösen des C wird der letzte Verschmelzungsschritt zu $S E \mathrm{Ni}_{2} \mathrm{~B}_{2} \mathrm{C}$ ebenfalls mehrfach wiederholt.

Die so präparierten 1-2 g schweren $S E \mathrm{Ni}_{2} \mathrm{~B}_{2} \mathrm{C}$-Proben zeigen typischerweise einen Masseverlust von weniger als $1 \%$ relativ zur Einwaage. Aus den so hergestellten polykristallinen Schmelzlingen können - nach einer Temperbehandlung - in einer Funkenerosionsanlage Proben gewünschter Form für Widerstands-, Suszeptibilitäts-, spezifische Wärmeoder Dilatationsmessungen präpariert werden ([Cam95], [Ove95], [Kno96], [Fü198]).

\subsubsection{Einkristallzucht}

Für die EK-Zucht werden $S E \mathrm{Ni}_{2} \mathrm{~B}_{2} \mathrm{C}$-Proben mit der gleichen Masse eines Flußmittels im Bornitridtiegel einer geigneten Wärmebehandlung unterzogen. Man verwendet in der Regel $\mathrm{Ni}_{2} \mathrm{~B}$ als Flußmittel $\left(T_{\mathrm{M}}=1100^{\circ} \mathrm{C}\right)$, da es gegenüber anderen Verbindungen den Vorteil hat, keine probenfremden Elemente zu enthalten. Bei jüngsten Einkristallzuchtversuchen an $\mathrm{CeNi}_{2} \mathrm{~B}_{2} \mathrm{C}\left(T_{\mathrm{P}} \leq 1200^{\circ} \mathrm{C}\right)$ hat sich aber auch das niederschmelzende CeNi $\left(T_{\mathrm{M}}=670^{\circ}\right)$ als Flußmittel bewährt [Pen00]. Den Tiegel gibt man mit einem Stück Zirkonfolie als Gettermaterial in einen Röhrenofen. So bestückt, heizt man den Röhrenofen unter ständigem Abpumpen ( $\mathrm{p} \leq 2 \cdot 10^{-5}$ mbar) oder ständigem Schutzgasdurchfluß (Ar; Reinheit 6.0) zunächst auf $200^{\circ} \mathrm{C}$, um eventuell von der Tiegel- oder Ofenwand desorbierende Adsorbate bei niedrigen Temperaturen aus dem Ofen zu treiben. Anschließend erwärmt man kontinuierlich bis knapp unter die peritektische Temperatur der SEBorkarbide $\left(S E\right.$-abhängig, $\left.T_{\mathrm{P}}(\mathrm{Ce}) \approx 1200^{\circ} \mathrm{C}, T_{\mathrm{P}}(\mathrm{Lu}) \approx 1500^{\circ} \mathrm{C}\right)$. Dabei schmilzt zunächst das Flußmittel und umgibt die eigentliche Probe, aus der bei einer ca. 5-stündigen Glühzeit bei $T_{\max }<T_{\mathrm{P}}\left(S E \mathrm{Ni}_{2} \mathrm{~B}_{2} \mathrm{C}\right)$ einkristalline Plättchen in das Flußmittel hineinwachsen.

Nach dem Glühen bei höchster Temperatur können die Einkristalle beim langsamen Abkühlen $\left(\mathrm{d} T / \mathrm{d} t=5^{\circ} \mathrm{C} / \mathrm{h}\right)$ weiter wachsen. Bei etwa $1000^{\circ} \mathrm{C}$ wird der Ofen zum raschen Abkühlen auf Raumtemperatur abgestellt. Nach der Entnahme der Probe werden mittels einer Funkenerosionsanlage die flächenhaft gewachsenen einkristallinen Plättchen aus dem umgebenden Flußmittel präpariert. Die Entfernung von polykristallinen Resten erfolgt durch anschließendes mechanisches Polieren. 


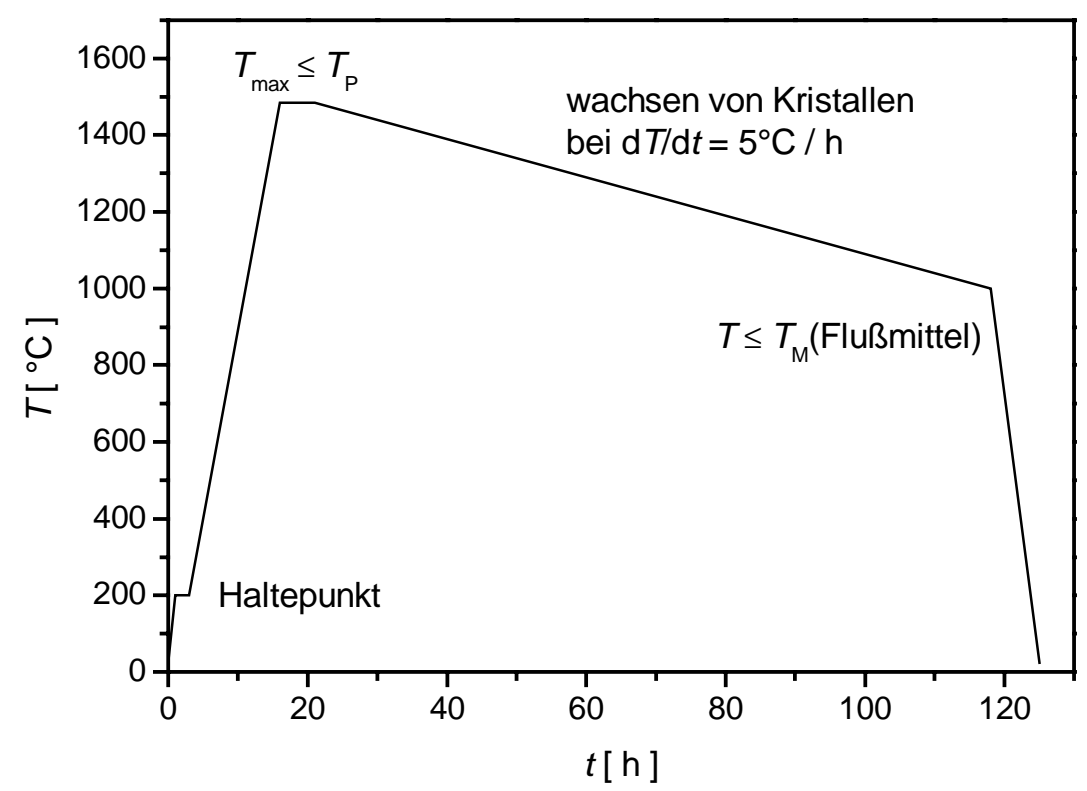

Abb.2.1 Typischer zeitlicher Verlauf der Heiztemperatur bei Einkristallzuchtversuchen der hier untersuchten Verbindungen $\mathrm{LuNi}_{2} \mathrm{~B}_{2} \mathrm{C}$ und $\mathrm{DyNi} \mathrm{i}_{2} \mathrm{C}$.

Mit diesem Verfahren ist es gelungen, $S E \mathrm{Ni}_{2} \mathrm{~B}_{2} \mathrm{C}$-Einkristalle mit Abmessungen bis 4.4.0.5 $\mathrm{mm}^{3}$ und Massen bis ca. $12 \mathrm{mg}$ zu präparieren. Die Mehrzahl der Proben liegen aber im Größenbereich von ca. 1.5 1.5 $0.3 \mathrm{~mm}^{3}$ und Massen um $1 \mathrm{mg}$. Speziell für die in Kap.5 beschriebenen Cantileverexperimente wurden äußerst dünne einkristalline Plättchen mit Massen z.T. unter $100 \mu \mathrm{g}$ und kreisförmigem Querschnitt $(\varnothing<1 \mathrm{~mm})$ präpariert.

\subsection{Probencharakterisierung}

Die entscheidenden Kriterien zur Charakterisierung der Probenqualität sind die Restwiderstandsverhältnisse und die Sprungtemperaturen $T_{\mathrm{c}}$ der Proben. Zur Bestimmung dieser Größen dienten Messungen des spezifischen Widerstandes im Temperaturbereich zwischen $300 \mathrm{~K}$ und $4 \mathrm{~K}$ ohne äußeres Magnetfeld.

\subsubsection{Widerstandsmessung en}

Abb.2.2 zeigt die Temperaturabhängigkeiten der besten in unserer Arbeitsgruppe präparierten $\mathrm{LuNi}_{2} \mathrm{~B}_{2} \mathrm{C}$ - und $\mathrm{DyNi}_{2} \mathrm{~B}_{2} \mathrm{C}$-Einkristalle (Verlauf für den $\mathrm{DyNi}_{2} \mathrm{~B}_{2} \mathrm{C}$-Einkristall nach [Pen99a]). Man sieht neben den scharfen Übergängen zur Supraleitung bei $T_{\mathrm{c}}\left(\mathrm{LuNi}_{2} \mathrm{~B}_{2} \mathrm{C}\right)=16.3 \mathrm{~K}$ und $T_{\mathrm{c}}\left(\mathrm{DyNi}_{2} \mathrm{~B}_{2} \mathrm{C}\right)=6.8 \mathrm{~K}$ im Dy-System noch die kontinuierliche Widerstandsabnahme unterhalb von $T_{\mathrm{N}}=10.3 \mathrm{~K}$ durch Abnahme der Spindisorder- 


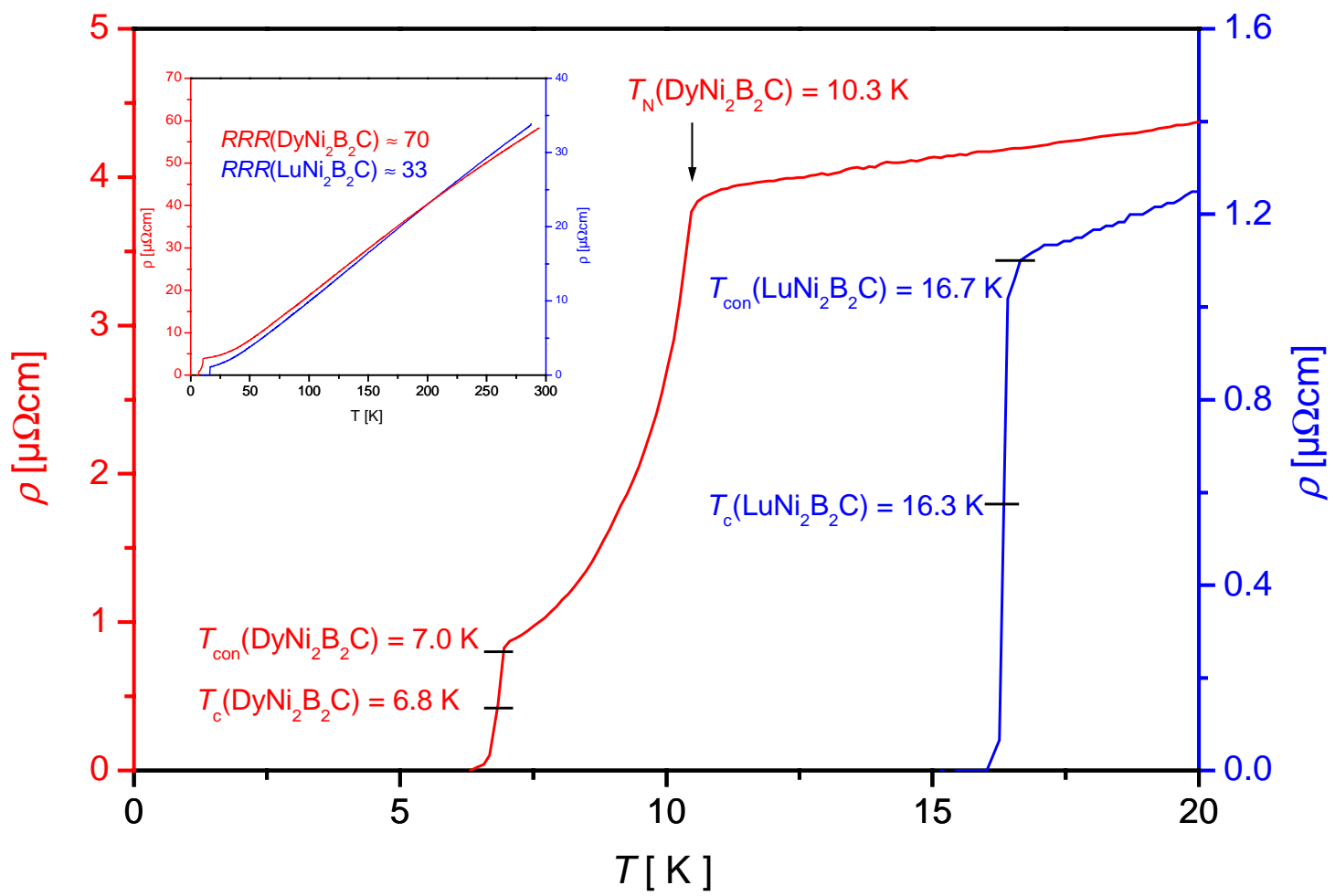

Abb.2.2 Temperaturabhängigkeit des spezifischen Widerstandes für Lu- und DyNi ${ }_{2} \mathrm{~B}_{2} \mathrm{C}$-Einkristalle unterhalb $300 \mathrm{~K}$.

Streuung im afm Zustand [Gsc82]. Die Restwiderstandsverhältnisse $R R R=\rho(300 \mathrm{~K}) / \rho\left(T_{\text {con }}\right)$ und Restwiderstände $\rho\left(T_{\text {con }}\right) \approx 1 \mu \Omega \mathrm{cm}$ der hier gezeigten Proben stellen die momentane obere Qualitätsgrenze dar

Alle in dieser Arbeit aufgenommen Widerstandsmessungen erfolgten im 4Kontaktverfahren mittels Lock-In-Technik ${ }^{1}$ bei typischen Modulationsströmen $I_{\text {eff }}=1 \mathrm{~mA}$ und Modulationsfrequenzen $\omega_{\mathrm{Mod}}=117 \mathrm{~Hz}$. Die Kontaktierung erfolgte über dünne Golddrähtchen $(\varnothing 25 \mu \mathrm{m})$, die mit Leitsilber auf die plättchenhaften Proben aufgeklebt wurden. Die so ermittelten Probenspannungen sind proportional zum Probenwiderstand. Aufgrund der kleinen Proben und der damit verbundenen Unsicherheit in der genauen Bestimmung der Kontaktgeometrie ist eine Berechnung des spezifischen Widerstandes $\rho(T)$ aus dem gemessenen $R(T)$ stark fehlerbehaftet (siehe [Kru96]). Die Umrechnung der Probenspannungen in spezifische Widerstände erfolgte daher unter Annahme

\footnotetext{
${ }^{1}$ Stanford SRS 850 oder EG\&G 5210
} 
der Gültigkeit der Matthiesenschen Regel [Ash76] für die hier untersuchten metallischen Proben:

Gl.2.1 $\rho=\rho_{0}+\rho_{p h}(T)+\rho_{\text {mag }}(T)$

Demnach tragen Beiträge aus verschiedenen Mechanismen der Elektronenstreuung additiv zum spezifischen Widerstand bei. Speziell sollte der neben dem magnetischen $\rho_{\text {mag }}$ und dem Restwiderstandsbeitrag $\rho_{0}$ auftretende phononische Beitrag $\rho_{\text {ph }}$ probenunabhängig sein. Durch Bestimmung von $\rho_{\mathrm{ph}}$ an polykristallinen Proben mit wohlbekannter Kontaktgeometrie (bspw. $\rho_{\mathrm{ph}}\left(\mathrm{LuNi}_{2} \mathrm{~B}_{2} \mathrm{C}\right)=33 \mu \Omega \mathrm{cm}$ aus [Nau96], [Ku94]) kann der entsprechende Beitrag $U_{\mathrm{ph}}$ aus den Einkristallmessungen umskaliert und allgemein $\rho(T)$ berechnet werden. 


\section{$3 \mathrm{LuNi}_{2} \mathrm{~B}_{2} \mathrm{C}$}

\subsection{Vorarbeiten aus der Literatur}

Die erste der beiden im Rahmen dieser Arbeit untersuchten $S E \mathrm{Ni}_{2} \mathrm{~B}_{2} \mathrm{C}$-Verbindungen ist das System $\mathrm{LuNi}_{2} \mathrm{~B}_{2} \mathrm{C}$. Es ist neben $\mathrm{YNi}_{2} \mathrm{~B}_{2} \mathrm{C}$ und $\mathrm{ScNi}_{2} \mathrm{~B}_{2} \mathrm{C}$ einer der Typ II-Supraleiter in dieser Klasse $\left(T_{\mathrm{c}}=16.3 \mathrm{~K}, \kappa \approx 21\right)$, die aufgrund ihrer abgeschlossenen Valenzschale als $S E^{3+}$ in der Verbindung kein lokales magnetisches Moment besitzen. Zum Verständnis der Supraleitung auch in den magnetischen Borkarbiden mit $m(S E)>m($ Dy) sind diese unmagnetischen Substanzen prädestiniert.

Bandstrukturrechnungen $\mathrm{zu} \quad \mathrm{LuNi}_{2} \mathrm{~B}_{2} \mathrm{C}$ und $\mathrm{YNi}_{2} \mathrm{~B}_{2} \mathrm{C}$ und anderen unmagnetischen $S E(\ddot{\mathrm{UM}})_{2}$-Borkarbiden mit verschiedenen Verfahren ${ }^{2}$ zeigen trotz der schichtartigen atomaren Struktur der Borkarbide (siehe Abb.1.1 eine 3-dimensionale elektronische Struktur. Diese Verfahren ergeben übereinstimmend eine hohe Zustandsdichte an der Fermikante $N\left(E_{\mathrm{F}}\right)$, die hauptsächlich durch Ni-3d-Zustände gebildet wird, während die Anteile der anderen Konstituenten je nach Verfahren leicht variieren. Die hohe Zustandsdichte wird als Grund für das relativ hohe $T_{\mathrm{c}}$ interpretiert, verglichen mit den vor den Borkarbiden bekannten konventionellen Supraleitern $\left(\left(T_{\mathrm{c}}\left(\mathrm{YPd}_{2} \mathrm{~B}_{2} \mathrm{C}\right)=23 \mathrm{~K}\right.\right.$, ebenso $T_{\mathrm{c}}($ A15 Verbindungen $)=23 \mathrm{~K}$ [Buc93]).

Mattheis et al. [Mat94a] stellten die zusätzliche These auf, daß eine „ideale Symmetrie“ des $\mathrm{NiB}_{4}$-Tetraeders um die Ni-Plätze der Struktur die Supraleitung begünstige. Diese „,ideale Symmetrie“ mit Tetraederwinkeln um $109^{\circ}$ ist in $\mathrm{LuNi}_{2} \mathrm{~B}_{2} \mathrm{C}$ nahezu realisiert, was zum bestmöglichen Ausbilden von $\mathrm{A}_{1 \mathrm{~g}}$-Phononen der Boratome in $\mathrm{LuNi}_{2} \mathrm{~B}_{2} \mathrm{C}$ führe. Zusammen mit der erhöhten Zustandsdichte ermöglichten diese die Supraleitung in konventioneller Elektron-Phonon-Kopplung.

Die aus den Bandstrukturen konstruierten Fermiflächen [Kim95], [Sin96], [Dre99] werden aus vier die Fermienergie schneidenden Bändern gebildet und sind für beide Substanzen sehr ähnlich. Dabei bildet eines der Bänder eine sehr kompliziert zusammenhängende Fläche entlang der $c$-Richtung der Brillouinzone und könnte somit u.a. anisotrope Magnetowiderstandseffekte erzeugen. Im Detail ergeben sich mögliche Effekte in der gene-

\footnotetext{
${ }^{2}$ Local-Density-Aproximation (LDA), Linearized Muffin Tin Orbital (LMTO), oder ab initio Pseudopotential Methoden, eine Übersicht findet sich in [Dre99])
} 
ralisierten Suszeptibilität $\chi(k)$ durch ausgeprägtes Fermiflächennesting entlang der Richtung (0.600) der Brillouinzone [Rhe95].

Speziell die berechnete erhöhte Zustandsdichte $N\left(E_{\mathrm{F}}\right)$ ist durch Messungen der spezifischen Wärmekapazität der experimentellen Überprüfung zugänglich [Car94], [Hon94]. Die experimentellen Werte der Sommerfeldkonstanten $\gamma_{\exp } \approx 20 \mathrm{~mJ} /\left(\mathrm{molK}^{2}\right)$ für $\mathrm{LuNi}_{2} \mathrm{~B}_{2} \mathrm{C}$ und $\mathrm{YNi}_{2} \mathrm{~B}_{2} \mathrm{C}$ ergeben mit $\gamma_{\text {exp }}=(1+\lambda) \cdot \gamma_{\text {band }}=(1+\lambda) \cdot\left(\pi^{2} / 3\right) k_{\mathrm{B}}^{2} \cdot N\left(E_{\mathrm{F}}\right)$ einen Wert von $\lambda \approx 1$ für den Elektron-Phonon-Kopplungsparameter. Zusammen mit der experimentell beobachteten $T_{\mathrm{c}}$-Abnahme bei Abweichungen von den ,idealen“ Ni-BBindungsverhältnissen [Mat94a] spricht dies für konventionelle Supraleitung mit mittlerer Elektron-Phonon-Kopplungsstärke im Rahmen der BCS-Theorie.

Eine Reihe von Experimenten zeigen ausgeprägte Anisotropien, die auf Supraleitungsmechanismen wie anisotrope $s$-Wellen-Supraleitung [Eli60] oder $d$-Wellen-Supraleitung [Mak96], [Wan98] schließen lassen.

So widerspricht die in magnetfeldabhängigen Experimenten zur spezifischen Wärme beobachtete $\sqrt{B}$-Abhängigkeit der Sommerfeldkonstanten im supraleitenden Zwischenzustand dem von der $B C S$-Theorie vorhergesagten linearen Verlauf, was als Indiz für ein partielles Verschwinden der Energielücke im Modell unkonventioneller Kopplung interpretiert wird [Noh97]. Weitere Wärmekapazitätsmessungen finden ebenfalls Anzeichen für ein partielles Verschwinden der Energielücke verknüpft mit Anisotropien im supraleitenden Kopplungsparameter [Man00]. Mikroskopische Experimente mittels Rastertunnelmikroskopie [Wi197] und Neutronenstreuung [Esk97] [McP98] an verschiedenen Borkarbiden (Lu-, Y-, Er-, $\mathrm{TmNi}_{2} \mathrm{~B}_{2} \mathrm{C}$ ) ergeben quadratische Flußliniengitter und magnetfeldinduzierte Flußliniengitterübergänge. Diese können durch Anlehnung an ähnliche Effekte in Hochtemperatursupraleitern im Rahmen der $d$-Wellen-Supraleitung, aber auch im Bild von konventioneller Kopplung im Formalismus anisotroper Ginzburg-LandauTheorie beschrieben werden [Wil97].

Merkmale sowohl der anisotropen $s$-Wellen- als auch der $d$-Wellen-Theorie sind u.a. die mögliche Implementierung einer Anisotropie der Energielücke $\Delta(\vec{k})$ für die Quasiteilchenanregungen im supraleitenden Zustand. Speziell das mögliche partielle Verschwinden der Energielücke auf Teilen der Fermifläche wäre damit in den Borkarbiden denkbar. 
Vom Standpunkt der größeren möglichen Anisotropien und auch räumlich variablerer Kopplungsstärke im $d$-Wellenmodell wäre die Existenz letzterer in den Borkarbiden natürlich besonders interessant.

Um weiteren Aufschluß über die Eigenschaften des Elektronensystems der Borkarbide zu gewinnen, wurden in dieser Arbeit de Haas-van Alphen (dHvA)-Experimente und anisotrope Widerstandsmessungen an $\mathrm{LuNi}_{2} \mathrm{~B}_{2} \mathrm{C}$-Einkristallen $(\mathrm{EK})$ durchgeführt.

Ziel der dHvA-Experimente, deren Grundlagen im nächsten Abschnitt 3.3.1 erläutert werden, ist es einen experimentellen Nachweis zumindest für Teile der aus Bandstrukturrechnungen konstruierten Fermiflächen zu erbringen. Dabei dürfen gemäß der Ähnlichkeiten in der Bandstruktur vergleichbare experimentelle Fermiflächenquerschnitte wie im Y-Borkarbid erwartet werden, bei dem bis $\mathrm{zu}$ acht dHvA-Signale $\mathrm{zu}$ verschiedenen Schnittflächen detektiert wurden [Ngu96]. Neben der möglichen prinzipiellen Überprüfung der berechneten Fermiflächen ist die Fragestellung interessant, in wie weit sich die in $\mathrm{YNi}_{2} \mathrm{~B}_{2} \mathrm{C}$-EK-Experimenten mögliche Beobachtung von dHvA-Oszillationen im supraleitenden Zustand auf das Lu-Borkarbid übertragen läßt. Unterhalb $B_{\mathrm{c} 2}$ können die Oszillationen gemäß vielfacher theoretischer Beschreibungen (siehe Abschnitt 3.3.2) nur bei teilweise verschwindender Energielücke existieren und sind an ersten Pulverexperimenten im $\mathrm{LuNi}_{2} \mathrm{~B}_{2} \mathrm{C}$ nicht beobachtet worden [Tok95].

Die durchgeführten Widerstandsmessungen zur Anisotropie in $B_{\mathrm{c} 2}$ dienen zur Überprüfung der vorgeschlagenen $d$-Wellen-Supraleitung in $\mathrm{LuNi}_{2} \mathrm{~B}_{2} \mathrm{C}$. Aus Magnetisierungsmessungen findet man eine Variation $B_{\mathrm{c} 2}(T, \alpha) \propto \cos (4 \alpha)$, wobei $\alpha$ der Winkel des angelegten Magnetfeldes in der $(a, b)$-Ebene relativ zur $a$-Achse des Kristalls ist [Met97]. Diese Winkelabhängigkeit von $B_{\mathrm{c} 2}$ ist im Bild isotroper $B C S$-Supraleitung nicht beschreibbar. Neben der in [Met97] vorgeschlagenen Beschreibung mit anisotroper $s$ Wellen-Theorie wurde von Maki et al. eine alternative Erklärung mittels $d$-WellenSupraleitung entwickelt [Wan98]. Die Motivation zur Anwendung derselben liegt dabei neben den zu beschreibenden $B_{\mathrm{c} 2}$-Anisotropien in einem Detail der berechneten Fermiflächen. Das Auftreten von Fermiflächennesting in den Borkarbiden [Rhe95] ist eine Gemeinsamkeit mit den mittels $d$-Wellen-Supraleitung beschriebenen Hochtemperatursupraleitern, bei denen Nestingkanäle entscheidenden Einfluß auf die Supraleitungskopplung zugeschrieben wird [Mak96]. 
Die Ausführungen von Maki et al. zur $B_{\mathrm{c} 2}$-Anisotropie in $\mathrm{LuNi}_{2} \mathrm{~B}_{2} \mathrm{C}$ ergeben die experimentell beobachtete Abhängigkeit $B_{\mathrm{c} 2}(T, \alpha) \propto \cos (4 \alpha)$ in der $(a, b)$-Ebene des Systems [Wan98]. Eine mögliche Überprüfung der Beschreibung der $B_{\mathrm{c}^{2}}$-Anisotropie mit den Parametern aus dem $d$-Wellen-Supraleitungsmodell ist die Ausweitung der experimentellen Daten zu tieferen Temperaturen. Dies erfolgte im Rahmen dieser Arbeit und wird in Kap.3.4 dargestellt.

Damit die für die Anisotropien erforderlichen Effekte beobachtet werden können, müssen Proben mit guter Qualität vorliegen. Die Bedingung von Supraleitung im „clean limit““ mit viel größeren mittleren freien Weglängen $l^{*}$ der Elektronen als der Ginzburg-LandauKohärenzlänge $\xi_{\mathrm{GL}}$ der Cooperpaare kann für die beste untersuchte Probe durch dHvAExperimente gewährleistet werden.

\subsection{Meßmethoden}

Änderungen der dHvA-Magnetisierung als auch die diamagnetische Suszeptibilität im supraleitenden Zustand wurden mit einer induktiven Meßmethode bestimmt. Die plättchenförmige Probe befindet sich im Inneren einer abgeglichenen Detektorspule, welche Änderungen der Probenmagnetisierung als Induktionsspannung mißt (Abb.3.1). Um tiefe Probentemperaturen zu gewährleisten, befindet sich das Detektorspulensystem in einem ${ }^{3} \mathrm{He}-\mathrm{Bad}$, welches in einem abgeschlossenem ${ }^{3} \mathrm{He}$-Gaskreislauf durch Dampfdruckerniedrigung auf $T=0.35 \mathrm{~K}$ abgekühlt werden kann. Die erforderlichen hohen und homogenen Magnetfelder am Probenort werden durch einen supraleitenden 12 T-Magneten erzeugt, der wie der ${ }^{3} \mathrm{He}$-Einsatz in einen ${ }^{4} \mathrm{He}$-Badkryostaten eingelassen ist [Gey87]. Die Probenorientierung relativ zum angelegten Magnetfeld kann über eine in das Gewinde des Probenträgers eingreifende Drehstange variiert werden. Dabei ist zu beachten, daß ein abnehmender Probenquerschnitt relativ zur Spulenachse das Signal verkleinert und dadurch die Meßbedingungen verschlechtert. Speziell bei den in der $(a, b)$-Ebene plättchenhaft gewachsenen $\mathrm{LuNi}_{2} \mathrm{~B}_{2} \mathrm{C}$-Einkristallen (siehe Kap.2) verringert sich der meßbare Probenquerschnitt bei Verkippung der $c$-Achse aus der Magnetfeldrichtung.

Die Suszeptibilitätsmessungen für feste Orientierung $B \|[001]$ und $T>4.2 \mathrm{~K}$ erfolgten in einem ${ }^{4} \mathrm{He}$-Badkryostaten mit ähnlichem auf dem Induktionsgesetz basierendem Detektorsystem [Bit97], [Kru96]. 
(a)

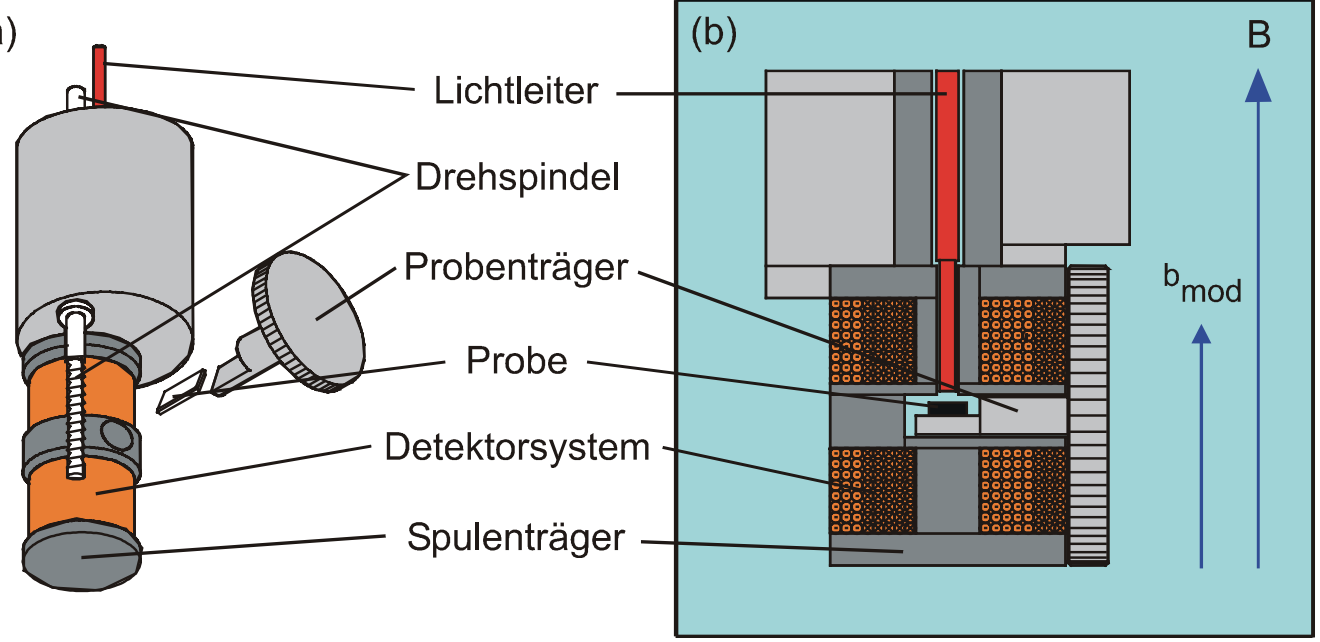

Abb.3.1 Im ${ }^{3}$ He-Kryostaten verwendetes Detektorspulensystem in Aufsicht (a) und im Schnitt (b). Die Besonderheiten gegenüber anderen Systemen sind die Möglichkeit zur Temperaturmodulation und das feststehende Detektorsystem bei Änderung der Probenorientierung (siehe auch [Hei95]).

Die von der Probenmagnetisierung erzeugten induktiven Spannungssignale sind i.a. kleine Signale von der Größenordung $\mu \mathrm{V}$ und werden mittels Lock-In-technischen Modulationsverfahren bestimmt.

Die dHvA-Magnetisierung zeigt eine ausgeprägte Magnetfeld- und Temperaturabhängigkeit, weshalb neben der üblichen Feldmodulationstechnik ein Temperaturmodulationsverfahren eingesetzt wurde (siehe Kap 3.3.3). Bei der Feldmodulationstechnik wird dem quasi statischen Magnetfeld $B$, von der Größenordnung einiger Tesla, ein paralleles niederfrequentes Wechselfeld $b_{\text {Mod }}=b_{0} \cdot \cos \left(\omega_{\text {Mod }} t\right)$ mit einer Amplitude $b_{0}$ im mT-Bereich überlagert. Bei der Temperaturmodulationsmethode wird die Probe über einen direkt oberhalb von ihr endenden Lichtleiter periodisch mit Laserlicht bestrahlt, was zu einer Oszillation $\Theta \cdot \cos \left(\omega_{\text {Mod }} t\right)$ um die mittlere Probentemperatur $T$ mit der Einstrahlfrequenz führt. Die gemessenen Signale sind also Induktionsspannungen der Art:

$$
\begin{aligned}
\text { Gl.3.1 } \quad U_{\text {Ind }} & \propto-\frac{d}{d t} \vec{M}\left(\vec{B}+\vec{b}_{0} \cos \left(\omega_{\text {Mod }} t\right), T\right) \cdot \vec{A}_{\text {Probe }} \quad b z w . \\
U_{\text {Ind }} & \propto-\frac{d}{d t} \vec{M}\left(\vec{B}, T+\Theta \cos \left(\omega_{\text {Mod }} t\right)\right) \cdot \vec{A}_{\text {Probe }}
\end{aligned}
$$


Diese werden über mehrere Perioden der Modulationsfrequenz $\omega_{\text {Mod }}$ oder im Feldmodulationsfall einer ihrer Oberwellen (siehe Kap.3.3.3 phasenempfindlich gleichgerichtet und zeitlich integriert.

Die Messung der magnetfeldabhängigen Wechselfeldsuszeptibilität $\chi_{\mathrm{ac}}$ B im supraleitenden Zustand basiert auf dem gleichen Lock-In-technischen Feldmodulationsverfahren, d.h.

Gl.3.2

$$
\begin{aligned}
U_{\text {Ind }} & \propto-\frac{d}{d t}\left(\vec{M}_{\text {dia }}\left(\vec{B}, \vec{b} \cos \left(\omega_{\text {Mod }} t\right)\right) \cdot \vec{A}_{\text {Probe }}\right) \\
& \propto-\omega_{\text {Mod }} \sin \left(\omega_{\text {Mod }} t\right) \cdot \chi_{\text {ac }} \cdot A_{\text {Probe }}
\end{aligned}
$$

Die durchgeführten Widerstandsmessungen erfolgten nach dem in Kap 2.2.1 angesprochenen 4-Kontaktverfahren mittels Lock-In-Technik. Die Experimente für feste Orientierung $B \|[001]$ und $T>4.2 \mathrm{~K}$ wurden in einem ${ }^{4} \mathrm{He}$-Badkryostaten durchgeführt, der ausführlich in [Kru96] beschrieben ist.

Zur Untersuchung der Winkelabhängigkeit $B_{\mathrm{c} 2}(T, \alpha)$ bei tiefster Temperatur wurde der in [Gey87] beschriebene ${ }^{3} \mathrm{He}-$ Kryostat verwendet, in dem auch die dHvA-Experimente stattfanden. Die Verkippung der Probe im umgebenden flüssigen ${ }^{3} \mathrm{He}$ bzw. für $T=4.2 \mathrm{~K}$ im flüssigen ${ }^{4} \mathrm{He}$ erfolgte wiederum über eine Drehspindel, die in das Außengewinde des die Probe tragenden Zylinders eingreift (siehe Abb.3.2.

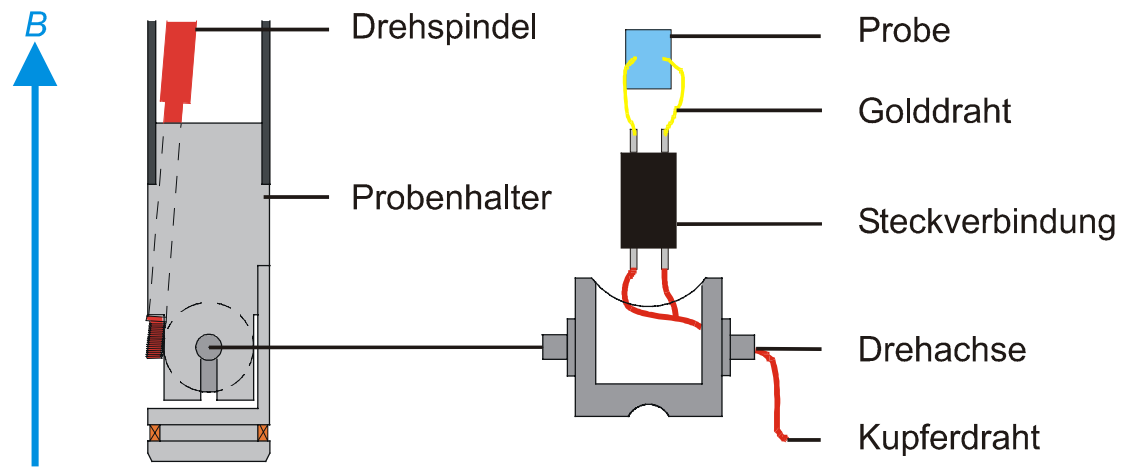

Abb.3.2 Im ${ }^{3}$ He-Kryostaten verwendete Widerstands-Meßsonde zur Bestimmung der Winkelabhängigkeit in $B_{\mathrm{c} 2}$.

\footnotetext{
${ }^{3}$ ac steht hier für alternating current und nicht für kristallographische Richtungen
} 


\subsection{Der de Haas-van Alphen-Effekt}

Die Fermiflächen der Energiebänder trennen bei $T=0 \mathrm{~K}$ die besetzten von den unbesetzten Festkörperelektronenzuständen im reziproken $k$-Raum. Nur an dieser Grenze liegen besetzte und unbesetzte Zustände mit vergleichbarem Impuls und Energien vor. Daher sind die Fermiflächen entscheidend für die niederenergetischen Anregungen oder Streuprozesse der Festkörperelektronen bspw. beim Ladungs- oder Wärmetransport. Ebenso erfolgt die Bildung von supraleitenden Cooperpaaren aus Zuständen nahe $E_{\mathrm{F}}$. Somit ist eine möglichst genaue Kenntnis der Fermifläche für die Beschreibung der Supraleitungseigenschaften nötig. Den direkten experimentellen Zugang liefert dabei der dHvA-Effekt [Kit88]. Die Theorie des oszillierenden dHvA-Signals basiert auf der Quantisierung der Elektronenzustände durch ein äußeres Magnetfeld in Landauniveaus [Lan30]. Sie wurde Anfang der 50er von Lifshitz und Kosevich entwickelt [Lif56] und erreichte in den 60ern experimentelle Bestätigung u.a. durch die Untersuchung der Fermiflächen der Edelmetalle mit dem dHvA-Effekt. Die Theorie, mögliche Meßmethoden zur Beobachtung und viele grundlegende Beispiele sind ausführlich in einem Buch von Shoenberg [Sho84] dargestellt. Weiterhin sind die im Rahmen dieser Arbeit zu seiner Beobachtung verwendeten Methoden seit einigen Jahren Standard in der Arbeitsgruppe Winzer und mehrfach beschrieben (u.a. [Gey87], [Thi92], [Hei95]), weshalb im nächsten Abschnitt nur auf die wesentlichen Ergebnisse der theoretischen Beschreibung und die experimentellen Besonderheiten eingegangen wird.

\subsubsection{Theorie}

Der dHvA-Effekt beschreibt die bei tiefen Temperaturen und hohen Magnetfeldern mögliche Beobachtung von Oszillationen einer elektronischen Meßgröße (elektronische Anteile der Wärmekapazität, Magnetisierung, Suszeptibilität) als Funktion des reziproken Magnetfeldes $1 / B$. Die Oszillationsfrequenz $F_{\mathrm{dHvA}}$ ist direkt proportional zu Extremalquerschnitten der Fermiflächen in der Ebene senkrecht zum angelegten Magnetfeld. Aus geeigneten orientierungs-, temperatur- oder feldabhängigen Experimenten kann auf die Geometrie der Fermifläche, die effektiven Massen $m$ oder mittlere Stoßzeiten $\tau$ der Elektronen geschlossen werden. Vom Standpunkt einer sich um die Fermienergie ausbildende Energielücke sind die 1976 erstmals beobachteten dHvA-Oszillationen im supraleitenden 
Zustand besonders spektakulär [Gra76]. Mögliche Erklärungen für das Auftreten des Effektes selbst bei Einsetzen der Supraleitung unterhalb $B_{\mathrm{c} 2}$ sind komprimiert in [Jan98] erläutert, worauf später in diesem Kapitel Bezug genommen wird.

Anschaulich ergibt sich folgendes Bild für den Grund der dHvA-Oszillationen [Kit88]: Ohne äußeres Magnetfeld werden die Festkörperelektronen als Blochfunktionen im periodischen Potential des Atomgitters beschrieben. Ihre Energieeigenwerte $E_{\mathrm{i}}\left(k_{\mathrm{x}}, k_{\mathrm{y}}, k_{\mathrm{z}}, s\right)$ ergeben sich aus den Quantenzahlen $k_{\mathrm{x}, \mathrm{y}, \mathrm{z}}=n \cdot\left(2 \pi / L_{\mathrm{x}, \mathrm{y}, \mathrm{z}}\right)$ im reziproken Raum, der Spinquantenzahl $s$, und dem Bandindex $i$, wobei $L_{\mathrm{x}, \mathrm{y}, \mathrm{z}}$ die Größe des Festkörpers in den drei Raumrichtungen beschreibt. Durch das Anlegen eines Magnetfeldes wird den Elektronen zwar im Mittel keine Energie zugeführt, aber in der Ebene senkrecht zum Feld wird ihre Bewegung auf Zyklotronbahnen der Frequenz $\omega_{\mathrm{c}}=\left(e B / m_{\mathrm{c}}\right)$ gelenkt. Die Definition einer effektiven Zyklotronmasse $m_{\mathrm{c}}$ folgt dabei aus der allgemeinen Verknüpfung der Energie $E$ der Elektronen bei der Zyklotronbewegung mit der dazugehörigen eingeschlossenen Fläche im $k$-Raum:

Gl.3.3 $m_{c}=\frac{\hbar^{2}}{2 \pi}\left(\frac{d S}{d E}\right)_{k \perp=k o n s t}$

Die nach der Bohr-Sommerfeldschen-Quantisierungsbedingung erlaubten geschlossenen Zyklotronbahnen ergeben quantisierte Energien für die Elektronen:

Gl.3.4 $\quad E=E_{k \|}+E_{k \perp}=\frac{\hbar^{2}}{2 m} k_{\|}^{2}+\hbar \omega_{c}(n+\gamma)$

Ein für große $L$ quasi kontinuierlicher und durch das $B$-Feld unveränderter Anteil $E_{\mathrm{k} \|}$ für die Bewegung entlang des Feldes und ein quantisierter Anteil $\hbar \omega_{c}(n+\gamma)$ in den sogenannten Landauniveaus mit $n \in N$ und $\gamma$ als Phasenkorrektur. Die Zyklotronbahnen, die mit der Zyklotronfrequenz $\omega_{\mathrm{c}}=\left(e \cdot B / m_{\mathrm{c}}\right)$ durchlaufen werden, schließen nach BohrSommerfeld im $k$-Raum senkrecht zum Magnetfeld die quantisierten Flächen 
Gl.3.5 $\quad S_{n}=\frac{2 \pi e B}{\hbar}(n+\gamma)$

ein. Da für bspw. $B \| e_{\mathrm{z}}$ alle Zustände $k_{\mathrm{x}, \mathrm{y}}$ jetzt durch Zustände in Landauniveaus beschrieben werden müssen, gilt mit der Dichte $d=\left(4 \pi^{2} / L_{\mathrm{x}} L_{\mathrm{y}}\right)$ der Zustände ohne Magnetfeld für die Entartung der Niveaus:

Gl.3.6 $\rho_{n}=\left(\frac{S_{n+1}-S_{n}}{\left(4 \pi^{2} / L_{x} L_{y}\right)}\right)=\left(\frac{2 \pi e B}{\hbar\left(4 \pi^{2} / L_{x} L_{y}\right)}\right)$

Man sagt, die vorher mit den Quantenzahlen $k_{\mathrm{x}, \mathrm{y}}$ beschrieben Elektronenzustände kondensieren in der Ebene senkrecht zum Magnetfeld auf den Landauniveaus $S_{\mathrm{n}}$.

Diese schneiden mit steigendem Feld sukzessive den Fermiflächenquerschnitt $A_{\mathrm{F}}$ in $\operatorname{der} k$ Ebene senkrecht zu $B$. Immer wenn gemäß Gl.3.5 $S_{\mathrm{n}}>A_{\mathrm{F}}$ ist, kommt es zur vollständigen Entleerung des Niveaus, da $E_{\mathrm{k} \perp}(n)>E_{\mathrm{Fermi}}\left(k_{\perp}\right)$ in dieser Ebene. Dies bewirkt eine Änderung der Gesamtenergie des Elektronensystems und den mit ihr über Gradientenbildung verbundenen Meßgrößen wie der Magnetisierung oder der Suszeptibilität. Gl.3.5 läßt darauf schließen, daß der Durchtritt benachbarter Niveaus $S_{\mathrm{n}}$ und $S_{\mathrm{n}+1}$ durch die konstante begrenzende Querschnittsfläche $A_{\mathrm{F}}$ periodisch mit der reziproken Feldgröße $\Delta(1 / B)$ erfolgt:

$$
\frac{1}{B_{n}}=\frac{2 \pi e}{\hbar}(n+\gamma) \frac{1}{S_{n}} \equiv \frac{2 \pi e}{\hbar}(n+\gamma) \frac{1}{A_{F}}
$$

Gl.3.7 $\frac{1}{B_{n+1}}=\frac{2 \pi e}{\hbar}(n+1+\gamma) \frac{1}{S_{n+1}} \equiv \frac{2 \pi e}{\hbar}(n+1+\gamma) \frac{1}{A_{F}}$

$$
\Rightarrow \Delta\left(\frac{1}{B}\right)=\frac{1}{B_{n}}-\frac{1}{B_{n+1}}=\frac{2 \pi e}{\hbar} \frac{1}{A_{F}}
$$

Die Frequenz $\Delta(1 / B)^{-1}$ des periodischen Durchtritts der Niveaus und der sich dabei ändernden Meßgrößen ist die dHvA-Frequenz

Gl.3.8 $\quad F_{\mathrm{dHvA}}=\frac{\hbar}{2 \pi e} A_{\mathrm{F}}$

die direkt proportional zum Fermiflächenquerschnitt $A_{\mathrm{F}}$ ist. 
Diese einfache Herleitung für das oszillierende dHvA-Signal beinhaltet bereits die wichtigsten experimentellen Anforderungen, die für seine Beobachtung zu erfüllen sind. Um den Elektronen eine nach Gl.3.4 gültige quantisierte Energie zuschreiben zu können, müssen sie eine endliche Verweildauer in den beschriebenen Landauniveaus besitzen. Dies führt zur Forderung nach reinen Proben und hohen Magnetfeldern in der sogenannten Zyklotronresonanzbedingung

Gl.3.9 $\quad \omega_{c} \tau>>1$,

wonach die Zahl der Zyklotronumläufe eines Elektrons groß gegen eins sein muß, bevor es nach der Stoßzeit $\tau$ aus dem Niveau gestreut wird. Außerdem muß die thermische Anregung der Elektronen sehr viel kleiner sein als die energetische Aufspaltung der Landauniveaus, d.h.

Gl.3.10

$$
\hbar \omega_{c}>k_{B} T
$$

All diese Ergebnisse sind in einer ausführlichen Herleitung für das thermodynamische Potential der Festkörperelektronen als Funktion des Magnetfeldes $B$ nach Lifshitz und Kosevich enthalten [Lif56]. Durch Gradientenbildung enthält man dort für den oszillierenden Anteil $\tilde{M}_{\|}$der parallel zum Magnetfeld gerichteten Magnetisierung der Festkörperelektronen folgenden Ausdruck:

\section{„Lifshitz-Kosevich(LK)-Formel“ für $\tilde{M}_{\|}$:}

$$
\tilde{M}_{\|}=\sum_{p} M_{0}(p) \sin \left(2 \pi p \frac{F_{d H v A}}{B}-\frac{1}{2} \pm \frac{\pi}{4}\right)
$$

Gl.3.11

$$
\begin{gathered}
=-\left(\frac{e}{\hbar}\right)^{3 / 2} \frac{\beta F B^{1 / 2} V}{2^{1 / 2} \pi^{5 / 2}\left(A^{\prime \prime}\right)^{1 / 2}} \sum_{p} R_{\mathrm{T}} R_{\mathrm{D}} R_{\mathrm{S}} \frac{1}{p^{3 / 2}} \sin \left(2 \pi p \frac{F_{d H v A}}{B}-\frac{1}{2} \pm \frac{\pi}{4}\right) \\
\beta=\frac{e \hbar}{m_{\mathrm{c}}} ; A^{\prime \prime}=\left(\frac{\partial^{2} A_{\mathrm{F}}}{\partial k_{\|}^{2}}\right)_{k e x t r} \quad ; p \in N
\end{gathered}
$$


Dabei ist auch hier die Periode der oszillierenden Magnetisierung durch die vorher definierte dHvA-Frequenz $F_{\mathrm{dHvA}}=(\hbar / 2 \pi e) A_{\mathrm{F}}$ bestimmt, allerdings folgt aus der allgemeinen Herleitung, daß man prinzipiell das dHvA-Signal jeder Oberwelle $p \cdot F_{\mathrm{dHvA}}$ beobachten kann, allerdings mit um den Vorfaktor $p^{(-3 / 2)}$ reduzierter Amplitude. Der Faktor $A^{\prime \prime}$ beschreibt die Krümmung der Fermifläche entlang der Feldrichtung in Höhe der Extremalebene $k_{\text {extr. }}$ Die Amplitudenreduktionsfaktoren $R_{\mathrm{T}}, R_{\mathrm{D}} \leq 1$ berücksichtigen die bereits oben motivierte Dämpfung des Effektes durch nicht ideale Versuchsbedingungen, wie das „Aufweichen“ der Fermifläche mit endlicher Temperatur $\left(R_{\mathrm{T}}\right)$ oder die erstmals von Dingle [Din52] berücksichtigte endliche Streuzeit $\tau$, die über die Unschärferelation eine störende Energieunschärfe bewirkt $\left(R_{\mathrm{D}}\right)$.

Gl.3.12 $\quad R_{T}=\frac{x}{\sinh (x)}$

mit $x=p \frac{2 \pi^{2} \mathrm{k}_{\mathrm{B}} T m}{e \hbar B}=p \cdot \lambda \frac{m}{m_{0}} \frac{T}{B} \quad$ und $\quad \lambda=\frac{2 \pi^{2} \mathrm{k}_{\mathrm{B}} m_{0}}{e \hbar}=14.693 \frac{\mathrm{T}}{\mathrm{K}}$

Gl.3.13 $R_{D}=\exp \left(-p \cdot \lambda \frac{m}{m_{0}} \frac{T_{\mathrm{D}}}{B}\right)$

Dabei ist die endliche Streuzeit $\tau$ über Naturkonstanten der Art

Gl.3.14 $\quad \tau^{-1}=\left(\frac{2 \pi \mathrm{k}_{\mathrm{B}}}{\hbar}\right) T_{\mathrm{D}}=0.82258 \cdot 10^{12} \frac{\mathrm{Hz}}{\mathrm{K}} \cdot T_{\mathrm{D}}$

mit der Dingletemperatur $T_{\mathrm{D}}$ verknüpft. Mit diesen beiden Amplitudenreduktionsfaktoren werden die bereits anschaulich eingeführten experimentellen Randbedingungen Gl.3.9 und Gl.3.10 auch formell erfaßt. Der Spindämpfungsfaktor $R_{\mathrm{s}}$ berücksichtigt die in Gl.3.4 vernachlässigte Energieaufspaltung der quantisierten Elektronenzustände für „Spin down“ und „Spin up“ Elektronen $\Delta \mathrm{E}= \pm 1 / 2 g \cdot \beta \cdot B$, wobei der Spinsplitting-Faktor i.a. durch $g=2$ genähert werden darf [Sho84]. Als Konsequenz erhält man eine zusätzliche Dämpfung des Signals 
Gl.3.15 $\quad R_{s}=\left(\cos p \frac{\pi}{2} g \frac{m}{m_{0}}\right)$.

Zur Vereinfachung wurde für alle drei Dämpfungsfaktoren $R_{\mathrm{T}, \mathrm{D}, \mathrm{S}}$ die gleiche effektive Elektronenmasse $m$ eingesetzt. Sie beinhaltet alle möglichen WW der Elektronen wie Elektron-Elektron-WW, Elektron-Phonon-WW und Bandeffekte. Streng gilt dies nur für $R_{\mathrm{T}}$, während beispielsweise in $R_{\mathrm{D}}$ die reine Bandmasse eingeht [Jan98]. Im allgemeinen ist die Bandmasse der gemessenen Fermiflächenquerschnitte allerdings nicht bekannt, so daß das aus $R_{\mathrm{T}}$ bestimmbare $m$ in guter Näherung in die anderen Dämpfungsfaktoren eingesetzt wird. Aus dieser quantitativen Beschreibung folgt, welche Eigenschaften der Festkörperelektronen mit dem dHvA-Effekt bestimmt werden können.

Man erhält über Gl.3.8 oder die LK-Formel die Größe von Extremalquerschnitten der Fermifläche $A_{\mathrm{F}}$ direkt aus der Frequenz eines als Funktion von $1 / B$ detektierten Meßsignales der Festkörperelektronen. Dabei kann die Frequenz entweder durch Fourieranalyse des dHvA-Signals oder durch Bestimmung der Steigung in einem sogenannten Landauplot erfolgen. Beim Landauplot wird die Vorstellung benutzt, daß während jeder Periode des dHvA-Signals ein Landauniveau den Fermiflächenquerschnitt schneidet. Trägt man nun Punkte gleicher Phase gegen $(1 / B)$ auf, so erhält man nach obigen Überlegungen einen linearen Zusammenhang für die innerhalb des Fermiflächenquerschnitts verbleibenden Landauzylinder. Die Steigung in einer solchen Auftragung ist demnach die dHvA-Frequenz

Gl.3.16

$$
n_{\text {Landau }}=\left[F_{\mathrm{dHvA}} / B\right]
$$

wobei [] für die „größte ganze Zahl“ steht und man unter $n_{\text {Landau }}$ die Zahl der vollständig besetzten Landauniveaus innerhalb des Fermiflächenquerschnitts versteht.

Durch Variation des Kippwinkels $\varphi$ von $B$ relativ zu den Probenachsen und Detektion einer möglicherweise veränderten dHvA-Frequenz erhält man Aussagen über die Orientierungsabhängigkeit der Fermiflächenquerschnitte. Wie aus G1.3.11 zu sehen, ist die Bestimmung der Orientierungsabhängigkeit auch durch die Periodizität des oszillierenden Signals bei konstantem Betrag aber kontinuierlicher Variation der Richtung des Feldes möglich: 
Gl.3.17 $\quad \tilde{M}_{\|}(\varphi)=M_{0}(\varphi) \sin \left(2 \pi \frac{F(\varphi)}{B}-\frac{1}{2} \pm \frac{\pi}{4}\right)$ mit $p=1$ in $\overline{G l .3 .11}$

Die winkelabhängige Amplitude ist dabei durch die Winkelabhängigkeit der Dämpfungsfaktoren begründet. Wird z.B. das winkelabhängige Signal einer Frequenz $F_{\mathrm{dHvA}}=1 \mathrm{kT}$ bei $|B|=10$ T detektiert, so erhält man aus der Lage benachbarter Maxima gemäß Gl.3.17 eine Änderung $F_{\mathrm{dHvA}}\left(\varphi_{1}\right)-F_{\mathrm{dHvA}}\left(\varphi_{2}\right)=10 \mathrm{~T}$. Die Genauigkeit dieses Verfahrens ist bei schwach ändernden Fermiflächenquerschnitten dem Verfahren durch Frequenzbestimmung mittels Fourieranalyse von dHvA-Signalen für verschiedene Orientierungen überlegen.

Aus der quantitativen Beschreibung der beobachteten Oszillationsamplitude mit der LKFormel Gl.3.11 folgen Aussagen über effektive Elektronenmassen $m(\varphi)$ und Stoßzeiten $\tau$ der Elektronen in den zugehörigen Fermiflächenquerschnitten.

Die Temperaturabhängigkeit von $M_{0}$ wird allein durch $R_{\mathrm{T}}$ bestimmt, so daß die Beschreibung der Amplitudenabnahme mit Gl.3.12 direkt auf die effektive Elektronenmasse $m$ führt.

Die Feldabhängigkeit von $M_{0}$ erlaubt Aussagen über $\tau$ für den untersuchten Fermiflächenquerschnitt. Die gemessene Oszillationsamplitude $M_{0}(B)$ des dHvA-Signals nach Gl.3.11 kann in 4 Faktoren zerlegt werden, von denen drei feldabhängig sind:

Gl.3.18 $\quad M_{0}(B)=M_{1} \cdot \sqrt{B} \cdot R_{\mathrm{T}}(B) \cdot R_{\mathrm{D}}(B)$

Durch die exponentielle Feldabhängigkeit von $R_{\mathrm{D}}$ (siehe Gl.3.13) ergeben sich die Dingletemperatur $T_{\mathrm{D}}$ bzw. die Stoßzeit $\tau$ als Steigung der halblogarithmischen Auftragung von der auf $R_{\mathrm{T}}(B) \cdot(B)^{1 / 2}$ normierten Amplitude für die Grundfrequenz mit $p=1$ :

Gl.3.19

$$
\ln \left(R_{\mathrm{D}}\right)=\ln \left(\frac{M_{0}(B)}{R_{\mathrm{T}} \cdot \sqrt{B}}\right)-\ln \left(M_{1}\right)=-\lambda \frac{m}{m_{0}} \frac{T_{\mathrm{D}}}{B}
$$

$$
\stackrel{(3.10)(3.11)}{\longrightarrow} \ln \left(M_{0}(B) \sinh \left[\lambda \frac{m_{\mathrm{c}}}{m_{0}} \frac{T}{B}\right](B)^{1 / 2}\right)=\text { konst. }-\lambda \frac{m}{m_{0}} \frac{T_{\mathrm{D}}}{B}
$$


Diese Auftragung der Amplitude nach Gl.3.19 wird als Dingleplot bezeichnet und führt mit Gl.3.13 auf die Stoßzeit $\tau$.

Prinzipiell kann die effektive Masse auch orientierungsabhängig bestimmt werden. Dazu kann in einer Messung bei konstantem Feld aber ändernder Feldorientierung Gl.3.15 dienen. Ein dort mögliches Auftreten von zusätzlich zu der durch $F(\varphi)$ begründeten Oszillationen, gemäß $\cos \left(m(\varphi) / m_{0}\right)$, führt auf die Winkelabhängigkeit der effektiven Masse. Diese ist im Rahmen der vorliegenden Arbeit allerdings nicht beobachtet worden und sei nur aus Gründen der Vollständigkeit erwähnt.

Bei der Amplitude des dHvA-Signales ist folgendes zu beachten: Sie ist über die Dämpfungsfaktoren $R_{\mathrm{T}}$ und $\mathrm{R}_{\mathrm{D}}$ empfindlich von der effektiven Masse $m$ der Elektronen im untersuchten Fermiflächenquerschnitt abhängig. Durch diese kann das Verschwinden eines dHvA-Signals für veränderte Orientierungen des Magnetfeldes relativ zur Probe durch eine Zunahme der effektiven Masse begründet sein. Ein weiterer möglicher Grund für das Verschwinden des dHvA-Signales nach einer Orientierungsänderung liegt in der Notwendigkeit einer geschlossen Begrenzung für den zu untersuchenden Fermiflächenquerschnitt. Ist diese Bedingung bei geänderter Magnetfeldrichtung nicht mehr erfüllt, so kann kein dHvA-Signal mehr detektiert werden.

Neben diesen in der Topologie der Fermifläche begründeten möglichen Dämpfungen für das dHvA-Signal gibt es noch einen von der Probengeometrie bestimmten Anteil. Wird wie bei hier durchgeführten Experimenten induktiv gemessen (siehe Kap.3.2, so ist die Querschnittsfläche senkrecht zur Spulenachse des verwendeten Detektorsystems entscheidend. Für kleinere Probenquerschnitte folgen mit dem Induktionsgesetz sofort kleinere Signalamplituden. 


\subsubsection{Der dHvA-Effekt im supraleitenden Zustand}

Die Beobachtung von dHvA-Oszillationen im supraleitenden Zustand erscheint vom Standpunkt bosonischer Cooperpaare und dem diamagnetischen Verhalten von Supraleitern zunächst unverständlich. Schließlich gilt die Theorie des dHvA-Effekts mit der LKTheorie für fermionische Festkörperelektronen und auch die diamagnetische Abschirmung spricht gegen eine mögliche Kondensation auf Landauniveaus durch angelegte äußere Magnetfelder. In Typ-II-Supraleitern mit sinkender diamagnetischer Abschirmung für $B>B_{\mathrm{c} 1}$ sollte jedoch zumindest nahe $B_{\mathrm{c} 2}$ das Eindringen des äußeren Magnetfeldes eine Quantisierung in Landauniveaus ermöglichen. Tatsächlich wird auch in einer Reihe von Typ-II-Supraleitern der dHvA-Effekt im supraleitenden Zustand beobachtet (siehe [Jan98] und Zitate darin). Unter Berücksichtigung einer für $B=B_{\mathrm{c} 2}$ verschwindenden Energielücke

Gl.3.20

$$
\Delta(B)=\Delta(B=0 \mathrm{~T}) \sqrt{1-\frac{B}{B_{c 2}(T=0 \mathrm{~K})}}
$$

[Bra88] ist die Existenz von fermionischen Quasiteilchenanregungen zumindest nahe $B_{\mathrm{c} 2}$ verständlich. Mit diesem Grundverständnis für die Beobachtbarkeit des Effekts auch im supraleitenden Zustand wurden eine Reihe von Theorien für seine quantitative Beschreibung auch unterhalb $B_{\mathrm{c} 2}$ entwickelt, die komprimiert in [Jan98] dargestellt sind. Das Ausbilden der Energielücke $\Delta$ kann nach obigen Ausführungen als Störung des dHvAEffekts bezeichnet werden. Dies wird in den theoretischen Beschreibungen des Effekts in einer zusätzlichen Streuzeit $\tau_{\mathrm{sl}}$ formell erfaßt, die im Dämpfungsterm $R_{\mathrm{D}}$ mündet:

Gl.3.21 $R_{D}(s l) \propto \exp \left(-\left(\frac{\text { konst }_{1}}{\tau_{0}}+\frac{\text { konst }_{2}}{\tau_{\mathrm{s} 1}}\right)\right)$

Die genaue Form, wie sich die Energielücke $\Delta$ auf die im supraleitenden Zustand zu erwartende zusätzliche Streuzeit $\tau_{\mathrm{sl}}$ überträgt, ist von Modell zu Modell verschieden. Einheitlich gilt aber für alle Beschreibungen, daß die Existenz von teilweise verschwindenden Energielücken, sogenannten ,gapless regions“ $\Delta(\vec{k})=0$, die Dämpfung minimieren sollte und dort somit $\omega \tau_{\mathrm{sl}}>>1$ gilt. 


\subsubsection{Meßprinzip}

Die sich ändernde dHvA-Magnetisierung wird mit einer induktiven Meßmethode als Suszeptibilitätsänderung bestimmt (siehe Abb.3.1. Das oszillierende dHvA-Signal ist klein gegenüber dem normalen diamagnetischen Anteil der Magnetisierung der Festkörperelektronen und wird daher Lock-In-technisch mit einem Modulationsverfahren gemessen. Die in Gl.3.11 abzulesende Temperatur- und Feldabhängigkeit der oszillierenden Magnetisierung ergibt die Möglichkeiten der Feld- oder Temperaturmodulation [Sho84].

\section{a) Feldmodulation}

Bei der Feldmodulationstechnik wird dem quasi statischen Magnetfeld $B$, von der GröBenordnung einiger Tesla, ein paralleles niederfrequentes Wechselfeld $b_{\text {Mod }}=b_{0} \cdot \cos \left(\omega_{\text {Mod }} t\right)$ mit einer Amplitude $b_{0}$ im mT-Bereich überlagert. Die oszillierende Magnetisierung aus der LK-Formel G1.3.11 ist somit eine Funktion von $B+b_{0} \cdot \cos \left(\omega_{\mathrm{Mod}} t\right)$

Gl.3.22 $\tilde{M} \stackrel{\text { Feldmod }}{\longrightarrow} \tilde{M}\left(B+b_{0} \cos \left(\omega_{\text {Mod }} t\right)\right)$

Das induktiv gemessene dHvA-Signal ergibt sich dann als Induktionsspannung zu

Gl.3.23 $\quad \tilde{U}_{\text {Ind }} \propto-\frac{d}{d t} \tilde{M}\left(B+b_{0} \cos \left(\omega_{\text {Mod }} t\right)\right)$

Die Magnetisierung kann für kleine Modulationshübe $b_{0}<<B$ entwickelt werden und aufgrund der nichtlinearen Abhängigkeit $\tilde{M}\left(B_{\text {ges }}\right)$ erhält man zu jeder Frequenz $F_{\mathrm{dHvA}}$ ein auf jeder Oberwelle $k \cdot \omega_{\text {Mod }}$ der Modulationsfrequenz oszillierendes Spannungssignal:

Gl.3.24 $\quad \tilde{U}_{\text {Ind }}=\sum_{k} U_{k \mathrm{dHvA}} \sin \left(k \omega_{\mathrm{Mod}} t\right)$

Gl.3.25 $\quad U_{k \mathrm{dHvA}}=-2 \omega_{\mathrm{Mod}} \cdot k \cdot k o n s t \cdot M_{0} \cdot J_{k}(y) \sin \left(\frac{2 \pi F_{d H v A}}{B}+\Phi+\frac{k \pi}{2}\right)$

Dabei ist die Amplitude $U_{\mathrm{k}}$ für jede Oberwelle $k \cdot \omega_{\text {Mod }}$ mit einer Spulenkonstanten, der Amplitude $M_{0}$ der Magnetisierung nach der LK-Formel und der Besselfunktion $k$-ter 
Ordnung beim Argument $y=\left(2 \pi \mathrm{F}_{\mathrm{dHvA}} \cdot b_{0} / B^{2}\right)$ gewichtet. Der Phasenfaktor $\Phi$ stammt aus der Phase in der LK-Formel. Da das Signal der Grundfrequenz $\omega_{\text {Mod }}$ durch die vom Modulationsfeld angeworfenen Abschirmströme feldabhängig gedämpft wird $\left(I_{\text {Ind }}=U_{\text {Ind }} / R_{\text {Probe }}(B)\right)$ wird i.a. das Meßsignal der ersten Oberwelle $2 \cdot \omega_{\text {Mod }}$ phasenselektiv Lock-In-technisch gleichgerichtet. Die im Ergebnisteil gezeigten dHvA-Signale entsprechen also der Induktionsspannung $U_{k \mathrm{dHvA}}$ für $k=2$.

Die Amplitudengewichtung mit der Besselfunktion 2. Ordnung hat dabei zwei wichtige Konsequenzen. Durch die zusätzliche Feldabhängigkeit $J_{2}\left(1 / B^{2}\right)$ muß die Bestimmung der Dingletemperatur nach Gl.3.19 modifiziert werden. Die dort auftretende Amplitude $M_{0}(B)$ der dHvA-Oszillationen ergibt sich zu $U_{k \mathrm{dHvA}}$ geteilt durch die speziell von der Feldmodulationstechnik bewirkte Feldabhängigkeit mit $J_{2}\left(1 / B^{2}\right)$ :

Gl.3.26 $\quad M_{0}(B)=\frac{U_{2 d H v A}}{J_{2}\left(\frac{2 \pi F b_{0}}{B^{2}}\right)}$

Die zweite wichtige Konsequenz wird aus der Darstellung des Betrags von $J_{2}$ für hier relevante Größen des Arguments $y$ deutlich. Das Verhältnis aus $b_{0} / B^{2}$ ist experimentell auf Größenordnungen $10^{-3} \mathrm{~T} /(5 \mathrm{~T})^{2}$ beschränkt. Damit wird der Verlauf von $J_{2}$ im wesentlichen durch die Größe der zu beobachtenden dHvA-Frequenz bestimmt.

Wie in Abb.3.3 zu sehen, ist die Beobachtung kleiner Frequenzen mit der Feldmodulationstechnik nur schwer oder gar nicht möglich, da die Lage des höchsten lokalen Maximums der Besselfunktion dann bei für den dHvA-Effekt ungünstig niedrigen Feldern liegt. 


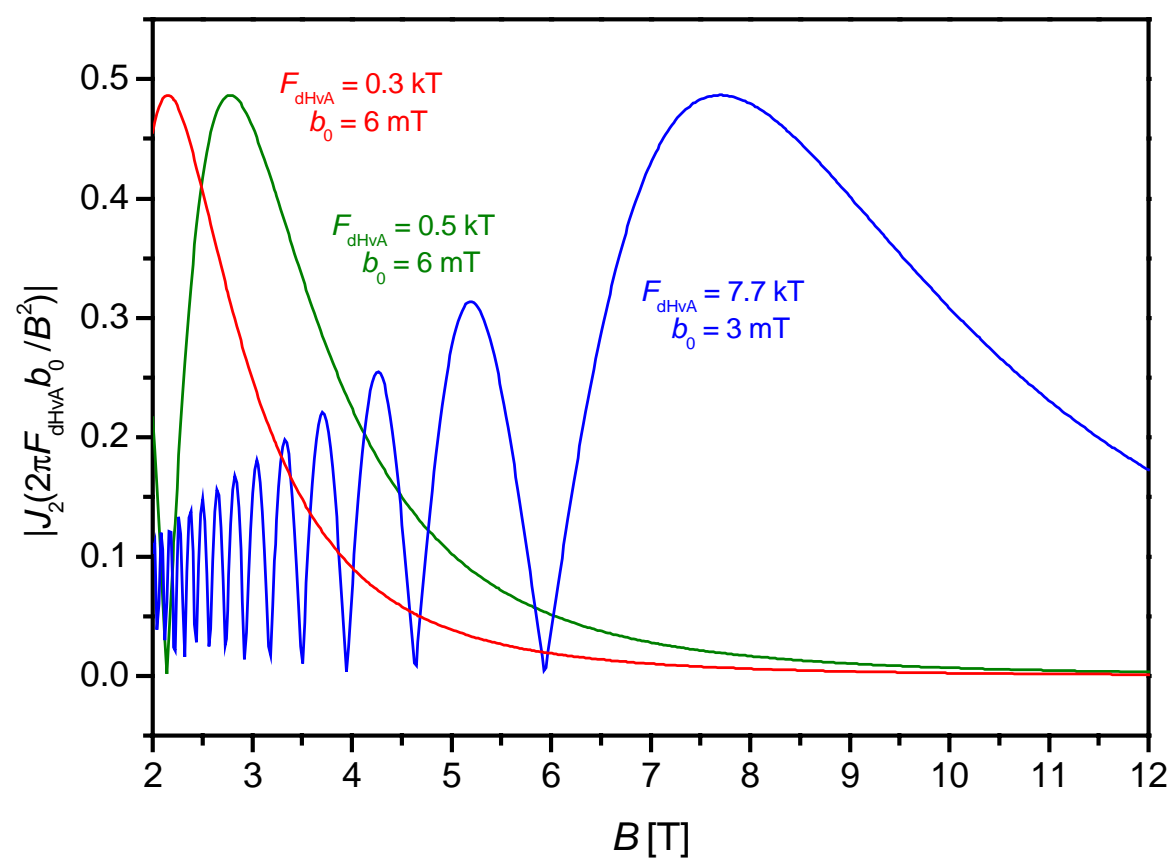

Abb.3.3 Verlauf des Betrages des bei der Feldmodulation zusätzlich auftretenden feldabhängigen Amplitudenfaktors $J_{2}$ für das dHvA-Signal.

\section{b) Temperaturmodulation}

Bei der Temperaturmodulationsmethode wird wie erwähnt die über $R_{\mathrm{T}}$ Gl.3.12 verknüpfte Abhängigkeit des dHvA-Signals nach der LK-Formel von der Temperatur ausgenutzt. Über einen direkt oberhalb der Probe endenden Lichtleiter (siehe Abb.3.1 kann diese periodisch mit Laserlicht bestrahlt werden. Für konstante eingestrahlte Laserleistung stellt sich eine mittlere Bad- und Probentemperatur ein, um welche die Probentemperatur mit dem Hub $\Theta$ periodisch oszilliert, d.h. $T_{\text {Probe }}=T_{\text {mitt }}+\Theta \cdot \cos \left(\omega_{\text {Mod }} t\right)$. Die Auswirkung dieser $T$-Modulation läßt sich am leichtesten beschreiben, wenn man den Beitrag jeder Frequenz $F_{\mathrm{dHvA}}$ bzw. $p \cdot F_{\mathrm{dHvA}}$ zur dHvA-Magnetisierung gemäß der LK-Formel in einen $T$-abhängigen $\left(R_{\mathrm{T}}\right)$ und einen $T$-unabhängigen Faktor $M_{2}$ aufspaltet:

Gl.3.27

$$
M(p)=M_{2} \cdot R_{\mathrm{T}}=M_{2} \cdot \frac{x}{\sinh x} \quad \text { mit } x=p \frac{2 \pi^{2} k_{\mathrm{B}}}{e \hbar} \frac{T m}{B} .
$$

Ersetzt man hier $T$ durch die oszillierende Probentemperatur, so ist $\mathrm{x}$ in G1.3.27 durch $z=x \cdot\left(1+\Theta / T \cdot \cos \left(\omega_{\text {Mod }} t\right)\right)$ zu ersetzen. Entwickeln des so geänderten Ausdrucks $M(p)$ 
für die im Experiment auftretenden kleinen Werte von $u=\Theta / T$ ergibt wie im Fall der Feldmodulation eine Reihe in Oberwellen $\cos \left(k \omega_{\text {Mod }} t\right)$ für $M\left(p, \cos \left(k \omega_{\text {Mod }} t\right)\right)$ und das Meßsignal $U_{\text {Ind }}$ :

Gl.3.28

$$
M(p) \propto \sum_{n=0} \frac{d^{n}}{d u^{n}}\left(\frac{z}{\sinh z}\right) u^{n} \propto \sum_{k=1} M\left(k \omega_{\mathrm{Mod}} t\right)
$$

Gl.3.29 $\quad \tilde{U}_{\text {Ind }} \propto-\frac{d}{d t} M(p)$

Die in der Entwicklung auftretende Reihe in Oberwellen $k \omega_{\text {Mod }}$ der Grundmodulationsfrequenz kann im Gegensatz zum Feldmodulationsfall leider nicht analytisch vereinfacht werden, wie etwa durch die dort auftretenden Besselfunktionen. Allerdings entsteht bei der Temperaturmodulationsmethode kein Dämpfungsterm, der selektiv auf einer der Frequenzen $k \omega_{\text {Mod }}$ wirkt wie die auf der Grundfrequenz dämpfende Abschirmung im Feldmodulationsfall. Daher wird hier i.a. auf die Grundfrequenz phasenselektiv gleichgerichtet und es gilt:

Gl.3.30 $\quad \tilde{U}_{\text {Ind }}=U_{\mathrm{dHvA}} \sin \left(\omega_{\mathrm{Mod}} t\right)$

Gl.3.31 $\quad U_{\mathrm{dHvA}}=-k o n s t \cdot \omega_{\mathrm{Mod}} \cdot M_{0} \cdot \frac{\Theta}{T} \cdot G\left(x, \frac{\Theta}{T}\right) \cdot\left(\frac{2 \pi F_{d H v A}}{B}+\Phi\right)$

Dabei ist $M_{0}$ wiederum die Amplitude des dHvA-Signals nach der LK - Formel und die feldabhängige Gewichtungsfunktion hat die Form:

Gl.3.32 $G\left(x, \frac{\Theta}{T}\right)=\frac{\sinh x}{x} \sum_{r=0} \frac{1}{r !(r+1) !} x^{2 r+1}\left(\frac{\Theta}{2 T}\right)^{2 r} \frac{d^{2 r+1}}{d x^{2 r+1}} \frac{x}{\sinh x}$

Da die Temperaturhübe $\Theta$ stets klein gegen die mittlere Temperatur sind kann dieser Ausdruck auf die erste Ordnung in $u=\Theta / T$ vereinfacht werden [Sho84]. Die im Ergebnisteil gezeigten dHvA-Signale sind Induktionsspannung $U_{\mathrm{dHvA}}$ der Art: 
Gl.3.33 $U_{\mathrm{dHvA}}=-k o n s t \cdot \omega_{\mathrm{Mod}} \cdot \frac{\Theta}{T} \cdot M_{0} \cdot\left(\frac{x}{\tanh (x)}-1\right) \cdot \sin \left(\frac{2 \pi F}{B}+\Phi\right)$

Der Faktor $(x / \tanh (x)-1)$ ist wiederum feldabhängig, so daß für die Bestimmung der Dingletemperatur aus der feldabhängigen dHvA-Amplitude analog zum Feldmodulationsfall die gemessene Amplitude gemäß

Gl.3.34 $\quad M_{0}(B)=\frac{U_{d H v A}}{\left(\frac{x}{\tanh (x)}-1\right)}$

zu modifizieren ist. Da der von der mittleren Temperatur beeinflußte Temperaturmodulationshub $\Theta(T)$ nicht experimentell zugänglich ist, kann bei diesen Messungen i.a. keine effektive Masse $m$ aus der T-Abhängigkeit der Oszillationsamplitude bestimmt werden [Thi92]. 


\subsubsection{Ergebnisse}

Im Folgenden werden die aus Messungen des dHvA-Effekts mit den erklärten $T$ - und $B$ Modulationsverfahren gewonnenen Ergebnisse präsentiert.

\subsubsection{T-Modulation}

Abb.3.4 zeigt ein mittels $T$-Modulation bei der mittleren Temperatur von $\langle T\rangle=0.77 \mathrm{~K}$ aufgenommenes dHvA-Signal in der Feldorientierung $B \|[001]+6^{\circ}$. Man erkennt deutlich die periodische Signalvariation mit der reziproken Feldgröße $B^{-1}$.
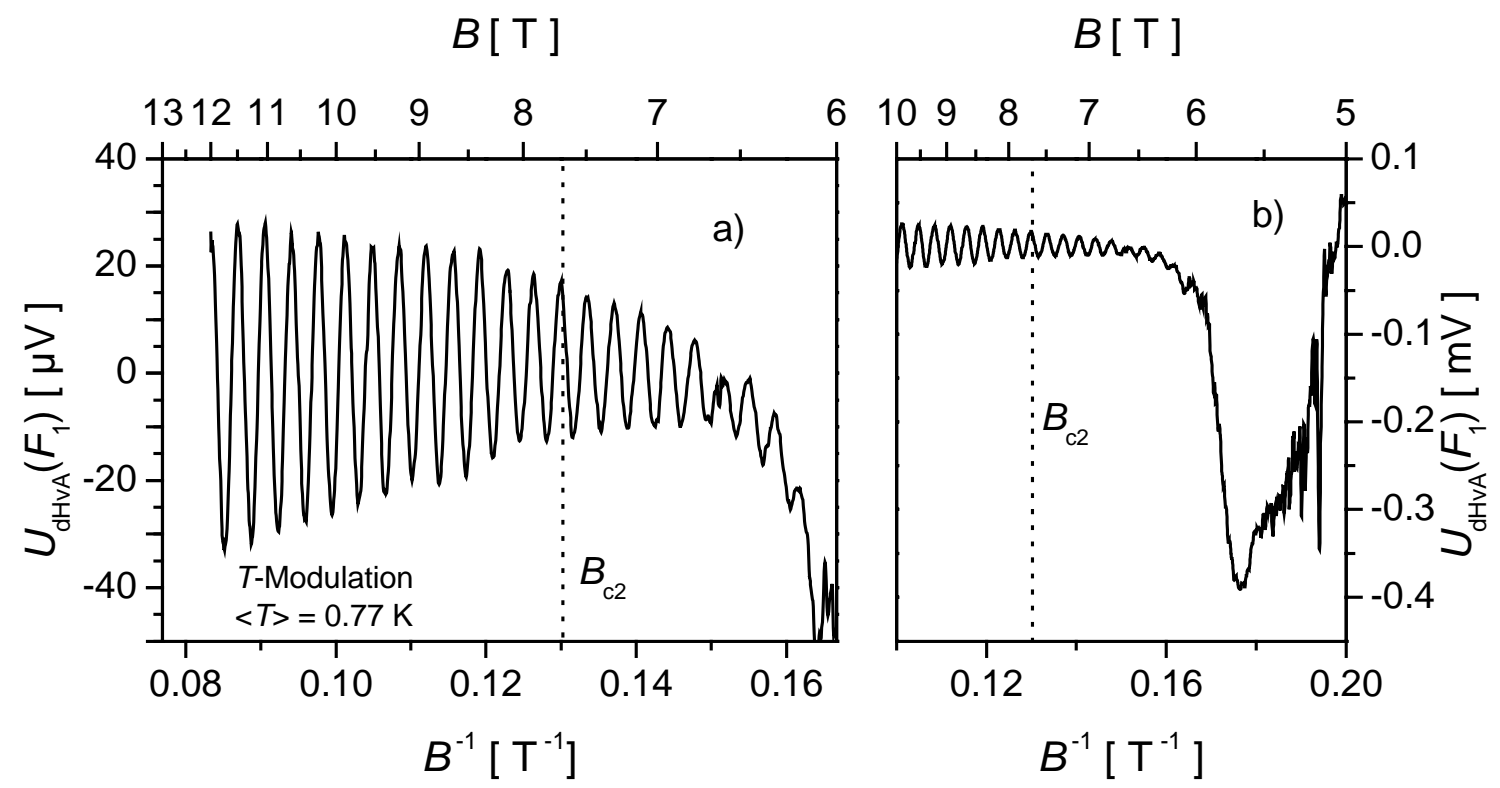

Abb.3.4 DHvA-Signal mittels T-Modulation in der Orientierung $B \|[001]+6^{\circ}$ im Feldbereich 13 6 (a) bzw. $10-5 \mathrm{~T}$ (b).

Die Oszillationsamplitude nimmt mit sinkendem Feld kontinuierlich ab, ohne daß beim Eintritt in den supraleitenden Zustand für $B=7.7 \mathrm{~T} \approx B_{\mathrm{c} 2}(0.8 \mathrm{~K}$ ) (siehe Kap.3.4) eine zusätzliche Dämpfung auftritt. Erst weit unterhalb $B_{\mathrm{c} 2}$ verschwinden die Oszillationen mit einer peakförmigen Signalabnahme bei Magnetfeldern $B_{\mathrm{c} 2} \geq 6.5 \mathrm{~T} \geq B \geq 5.5 \mathrm{~T}$, wobei unterhalb 5.5 T im wesentlichen nur noch Untergrundrauschen detektiert wird (siehe Abb.3.4b). Die Frequenzanalyse des Signals, dargestellt in Abb.3.5, ergibt eine dHvAFrequenz von $279.4 \pm 0.2 \mathrm{~T}$ was einem Fermiflächenquerschnitt $A_{1}=2.67 \pm 0.01 \mathrm{~nm}^{-2}$ gemäß Gl.3.8 entspricht. Die Auftragung der lokalen Maxima und Minima des Signals im 

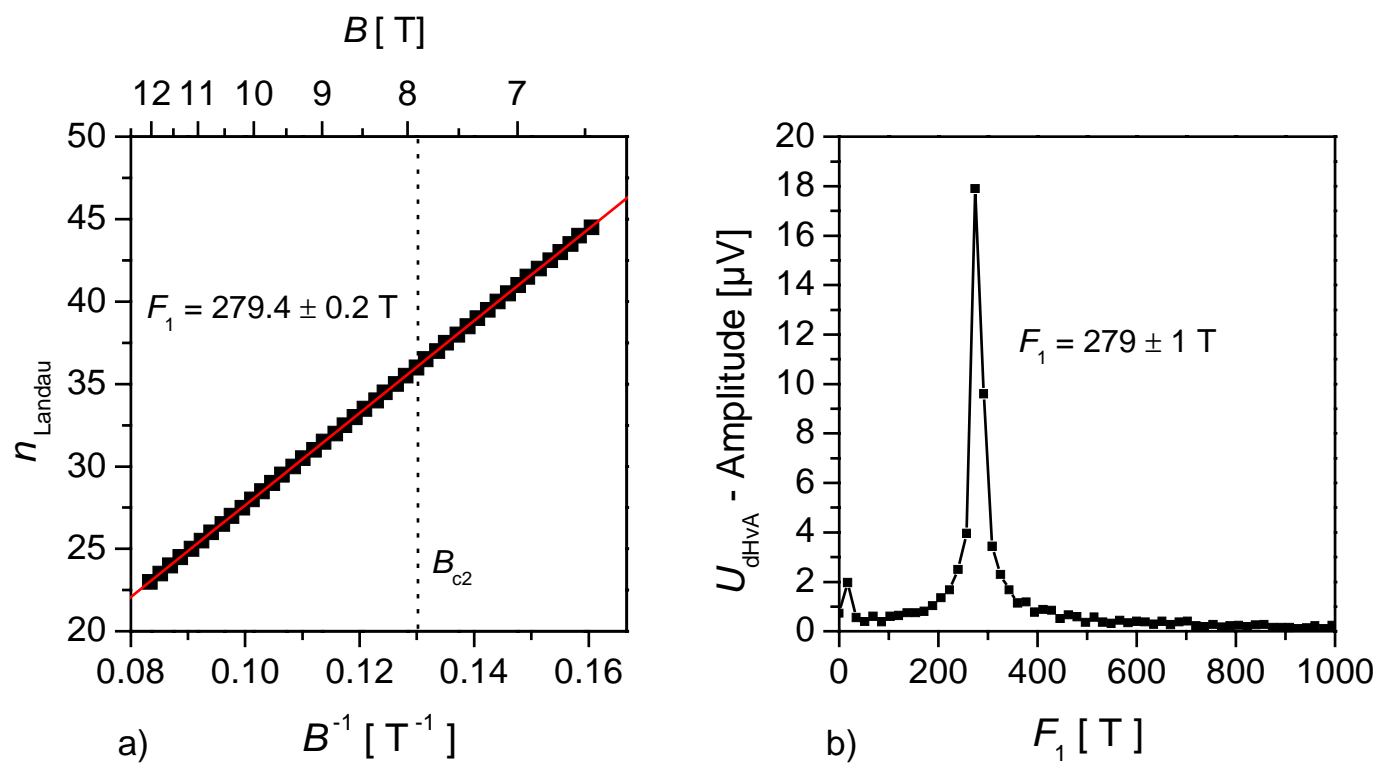

Abb.3.5 Frequenzanalyse der Messung aus 4bb.3.4. In a) mittels Landauplot und in b) mittels Fourieranalyse.

Landauplot ergibt einen streng linearen Verlauf, der auch unterhalb $B_{\mathrm{c} 2}$ nicht von der Steigung $F_{1}=279.4 \mathrm{~T}$ abweicht.

Wie in Kap.3.3.3 erwähnt kann aus Experimenten mit T-Modulation keine effektive Elektronenmasse ermittelt werden. Die über alle Orientierungen gemittelte effektive Masse des Fermiflächenquerschnitts $A_{1}$ ist allerdings aus Hochfeldexperimenten an $\mathrm{LuNi}_{2} \mathrm{~B}_{2} \mathrm{C}$-Pulver bekannt [Tok95]. Mit dem dort ermittelten $m_{1}=0.55 \cdot m_{0}$ ist eine Bestimmung der Fermigeschwindigkeit $v_{\mathrm{F}}$ und der Streuzeit $\tau$ im untersuchten Querschnitt möglich. Unter der Annahme fast freier Elektronen der effektiven Masse $m_{1}$ erhält man für die Fermigeschwindigkeit $v_{\mathrm{F} 1}=\sqrt{2 e \hbar F_{1}} / m_{1}=1.93 \cdot 10^{5} \mathrm{~m} / \mathrm{sec}$.

Zur Bestimmung der Streuzeit $\tau$ wird die feldabhängige Oszillationsamplitude $U_{\mathrm{dHvA}}$ gemäß Gl.3.19 und Gl.3.34 auf die feldabhängigen Vorfaktoren $R_{\mathrm{T}^{*}}(B)^{(1 / 2)} \cdot(x / \tanh (x)-1)$ normiert. Der Logarithmus dieser normierten Amplitude ist gerade $\ln \left(R_{\mathrm{D}}\right)$ zuzüglich einer Konstanten. Die Steigung in der Feldabhängigkeit von $\ln \left(R_{\mathrm{D}}\right)$ führt nach Gl.3.19 auf die Dingletemperatur $T_{\mathrm{D}}$ und die Streuzeit $\tau$. Die Anpassung des experimentellen Verlaufs von $\ln \left(R_{\mathrm{D}}\right)$ in Abb.3.6 ergibt eine Steigung von $-28 \pm 1$ T. Mit der effektiven Elektronenmasse $m_{1}=0.55 \cdot m_{0}$ erhält man demnach für die Dingletemperatur $T_{\mathrm{D}}=3.5 \pm 0.1 \mathrm{~K}$ und die Streuzeit der Elektronen beträgt $\tau_{0}=(0.35 \pm 0.01) \cdot 10^{-12} \mathrm{sec}$. 


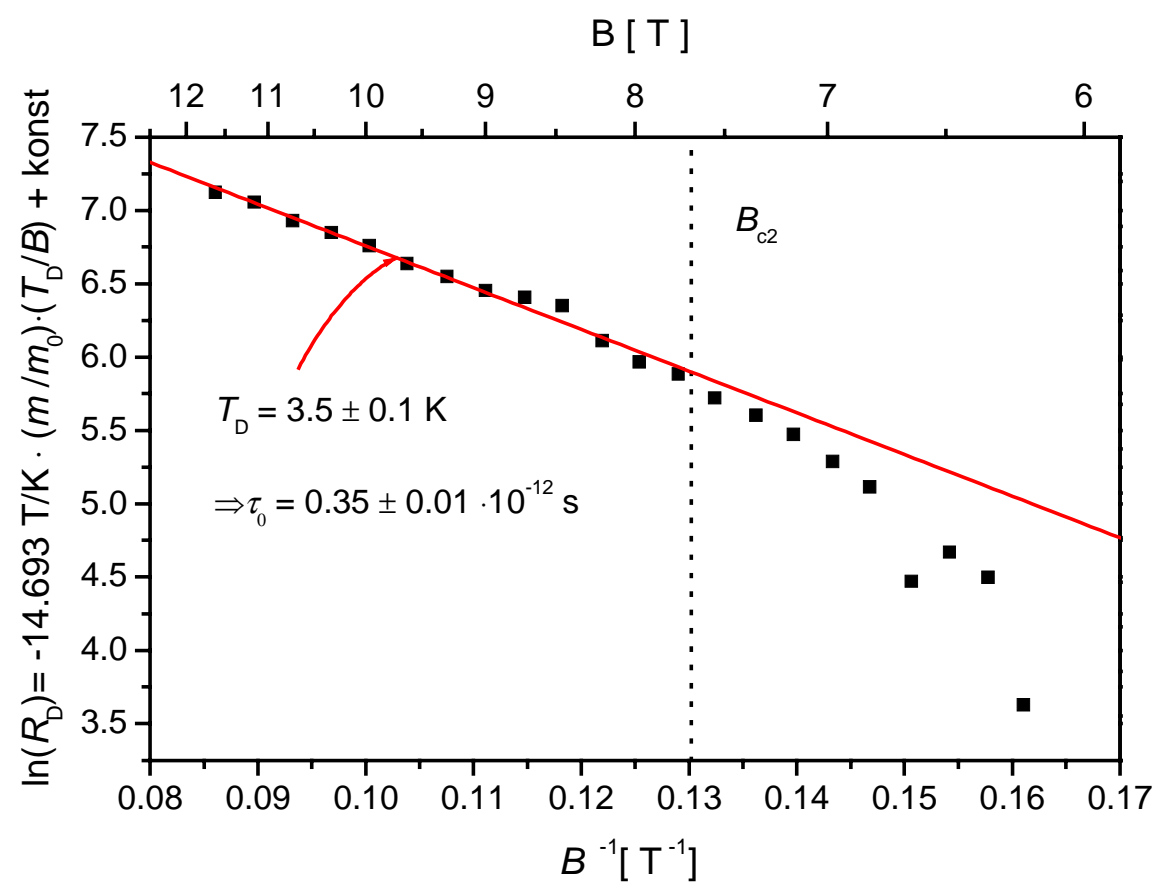

Abb.3.6 Bestimmung der Dingletemperatur $T_{\mathrm{D}}$ aus der Feldabhängikeit von $\ln \left(R_{\mathrm{D}}\right)$. Die schwarzen Symbole ergeben sich aus der Auswertung der Oszillationsamplitude einer Messung zwischen 12 und $8 \mathrm{~T}$ gemäß Gl.3.19 und Gl.3.34. Die rote Kurve entspricht der linearen Anpassung an die experimentellen Werte für $B>B_{\mathrm{c} 2}$.

Erst für Felder kleiner $B_{\mathrm{c} 2}$, bei $B \approx 6.5 \mathrm{~T}$, liegt der experimentelle Verlauf deutlich unterhalb der linearen Anpassung. D.h. erst dort tritt eine zusätzliche Dämpfung auf. Aufgrund der sehr kleinen Frequenz $F_{1}$ und des Verschwindens der Oszillationen bei Eintritt der peakförmigen Signaländerung können im Vergleich zum Y-System [Hei95] jedoch nur wenige Oszillationen unterhalb $B_{\mathrm{c} 2}$ beobachtet werden. Daher muß hier auf die Anpassung der zusätzlichen Dämpfung im supraleitenden Zustand nach Gl.3.21 verzichtet werden.

Außer $F_{1}$ konnten keine weiteren Frequenzen mittels $T$-Modulation beobachtet werden, so daß das Hauptaugenmerk auf der Charakterisierung der Winkelabhängigkeit von $F_{1}$ lag. Dazu wurden feldabhängige Oszillationen für verschiedene konstante Verkippungen des Magnetfeldes innerhalb der $(a, c)$-Ebene des Kristalls durchgeführt. Es konnten Oszillationen bis zu einem maximalen Verkippungswinkel $\varphi$ zwischen $B$ und [001] von ca. $50^{\circ}$ detektiert werden, wobei die Frequenz mit $\varphi$ ansteigt (quadratische Symbole in Abb.3.9. Am klarsten tritt die Winkelabhängigkeit eines Fermiflächenquerschnitts zum Vorschein,wenn man sie, wie in Kapitel 3.3.1 beschrieben, in einem Experiment bei 


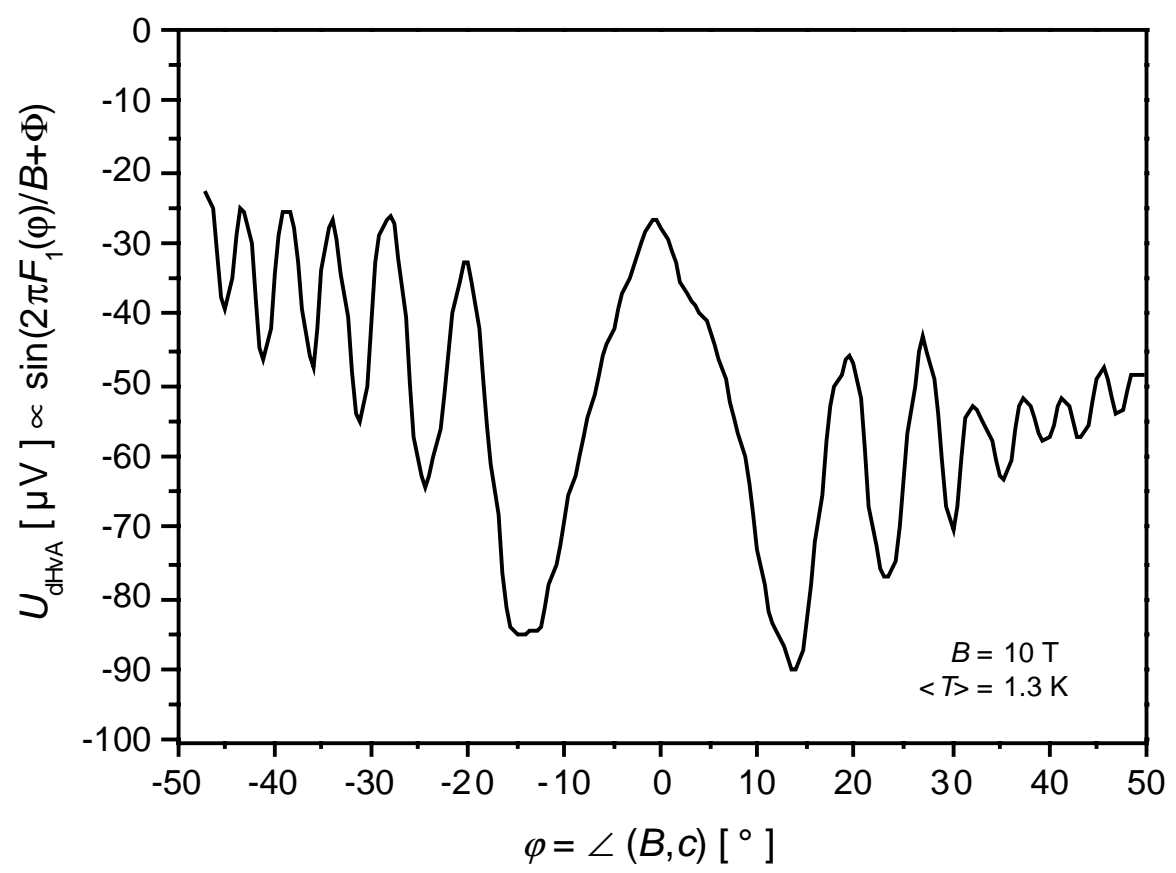

Abb.3.7 Orientierungsabhängiges dHvA-Signal innerhalb der (a,c)-Ebene.
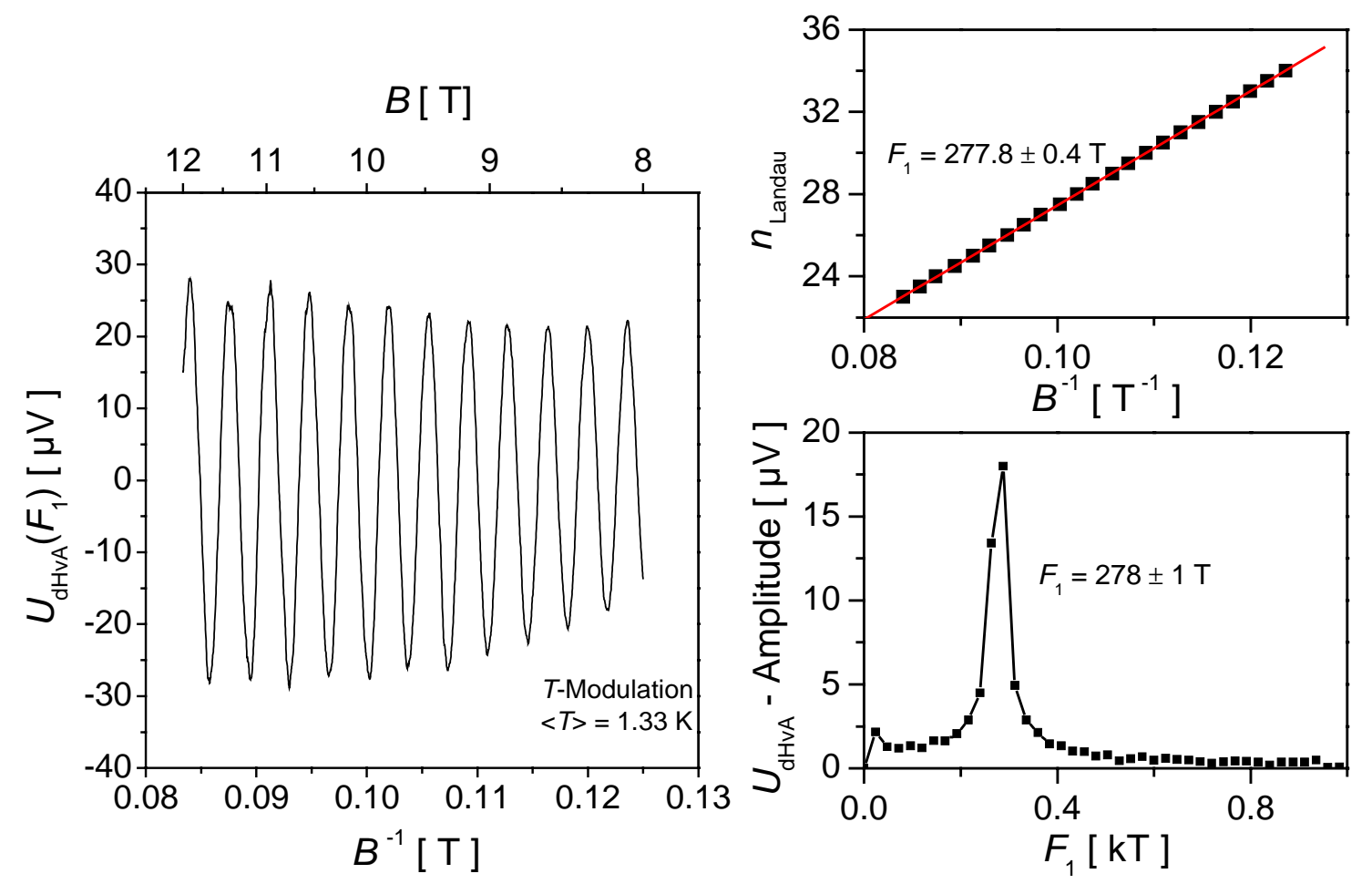

Abb.3.8 DHvA-Oszillationen in der Symmetrielage B || [001] mit zugehörigen Frequenzanalysen. 
konstantem Feldbetrag, aber kontinuierlicher Variation der Feldorientierung relativ zu den Kristallachsen beobachten kann. Abb.3.7 zeigt solch ein mittels T-Modulation aufgenommenes Signal bei einer mittleren Temperatur $\langle T\rangle=1.3 \mathrm{~K}$, konstantem Feldbetrag $B=10 \mathrm{~T}$ und kontinuierlicher Verkippung der Feldrichtung innerhalb der $(a, c)$-Ebene des $\mathrm{LuNi}_{2} \mathrm{~B}_{2} \mathrm{C}-\mathrm{EK}$. Man sieht deutlich die sinusförmige Signalvariation $U_{\mathrm{dHvA}} \propto \sin \left(2 \pi F_{1}(\varphi) / B+\Phi\right)$ gemäß G1.3.17, Die Abb.3.8 zeigt die Ermittlung der dHvAFrequenz in der Symmetrielage $B \|[001]$ aus Abb.3.7 durch ein Experiment $U_{\mathrm{dHvA}}\left(B, \varphi=0^{\circ}\right)$. Mit der Kenntnis $F_{1}\left(\varphi=0^{\circ}\right)=277.8 \pm 0.4 \mathrm{~T}$ und einer zunehmenden Frequenz für $\varphi>0^{\circ}$ ist deren Winkelabhängigkeit vollständig aus der Phase des Arguments $\left(2 \pi F_{1}(\varphi) / 10 \mathrm{~T}+\Phi\right)$ in Abb.3.7 bestimmbar. Sie ist in Abb.3.9 gemeinsam mit der aus einem Experiment $U_{\mathrm{dHvA}} \propto \sin \left(2 \pi F_{1}(\varphi) / 11 \mathrm{~T}+\Phi\right)$ und der aus den angesprochenen feldabhängigen Messungen gewonnen Winkelabhängigkeit dargestellt. Die experimentellen Meßwerte lassen sich mit der Annahme einer Fermifläche in Form eines Rotationsellipsoiden sehr gut analytisch beschreiben. Für eine rotationsellipsoide Fermifläche mit langer Achse $k_{\mathrm{c}}$ entlang [001] und kurzer Achse $k_{\mathrm{a}}$ in der $(a, b)$-Ebene gilt für Querschnittsflächen durch den Ursprung:

Gl.3.35

$$
A(\varphi)=\frac{A\left(0^{\circ}\right)}{\sqrt{\cos ^{2}(\varphi)+\left(\frac{k_{\mathrm{a}}}{k_{\mathrm{c}}}\right)^{2} \sin ^{2}(\varphi)}}
$$

Dabei ist $\varphi$ der Winkel zwischen der Normalen der Querschnittsflächen und der [001]Richtung. D.h. $A\left(\varphi=0^{\circ}\right)$ beschreibt die Querschnittsfläche, die für $B \|[001]$ im dHvAEffekt beobachtbar ist und $A\left(\varphi=90^{\circ}\right)$ ist der entsprechende Querschnitt für $B \|[100]$. Die in Abb.3.9 eingezeichnete grüne Kurve ist der nach Gl.3.35 angepaßte Verlauf mit dem Achsenverhältnis $\left(k_{\mathrm{a}} / k_{\mathrm{c}}\right)^{2}=0.399$. Für die Symmetrielagen gilt demnach:

$$
\begin{aligned}
& A\left(0^{\circ}\right)=\frac{2 \pi e}{\hbar} F_{1}\left(0^{\circ}\right)=\frac{2 \pi e}{\hbar} 278 \mathrm{~T}=\pi k_{\mathrm{a}}^{2}=2.65 \mathrm{~nm}^{-2} \text { und } \\
& A\left(90^{\circ}\right)=\frac{2 \pi e}{\hbar} F_{1}\left(90^{\circ}\right)=\frac{2 \pi e}{\hbar} 440 \mathrm{~T}=\pi k_{\mathrm{a}} k_{\mathrm{c}}=4.20 \mathrm{~nm}^{-2}
\end{aligned}
$$




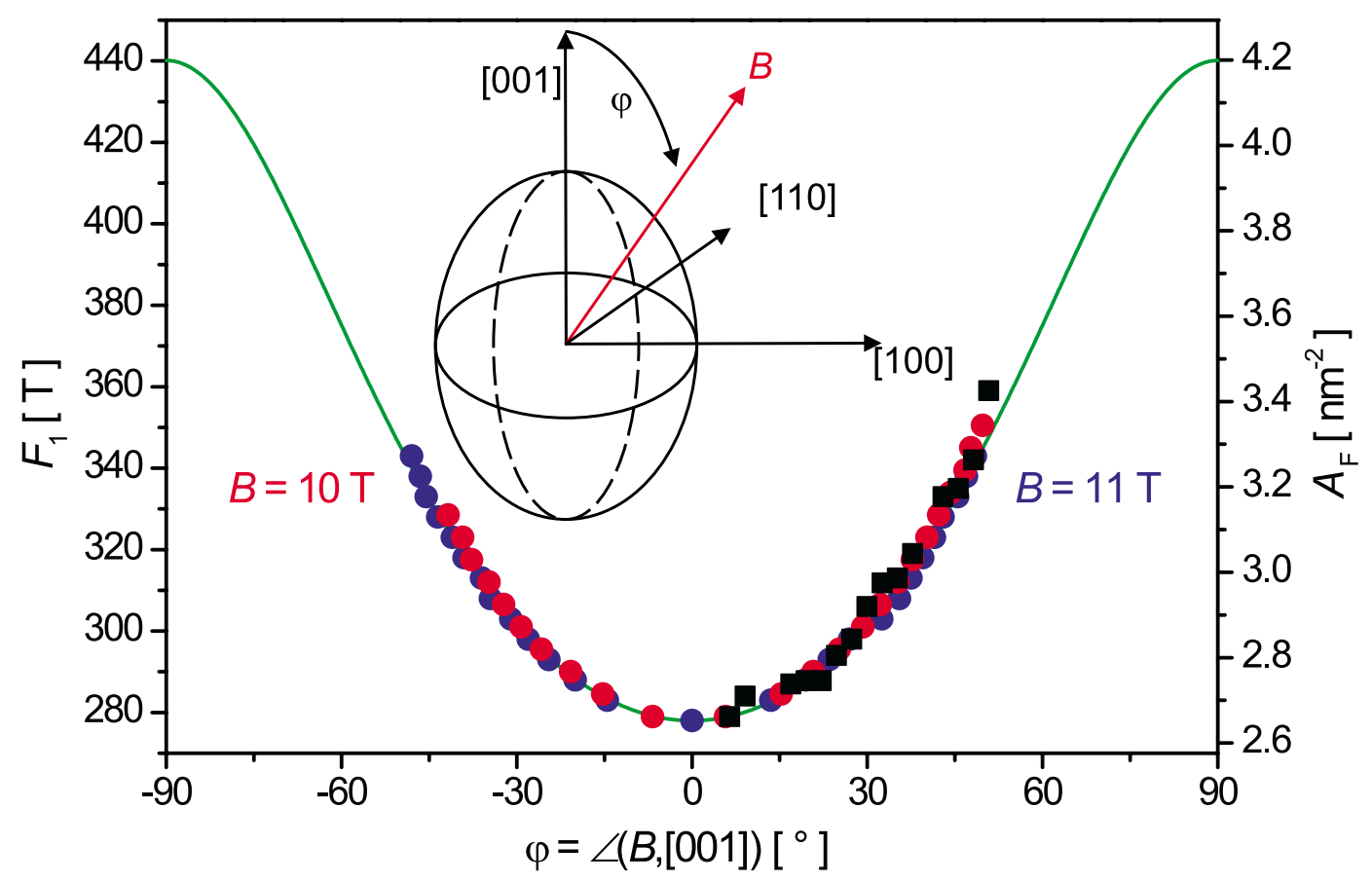

Abb.3.9 Winkelabhängigkeit der dHvA-Frequenz $F_{l}$ für B-Feldrichtungen innerhalb der (a,c)-Ebene.

\subsubsection{B-Modulation}

Abb.3.10 zeigt ein mittels $B$-Modulation bei $T=0.6 \mathrm{~K}$ aufgenommenes dHvA-Signal in der Feldorientierung $B \|[001]$. Die Signalamplitude von etwa $2 \mu \mathrm{V}$ ist sehr viel kleiner als die aus den Experimenten mit $T$-Modulation. Dies liegt an der im Feldmodulationsfall nötigen Einschränkung auf Modulationsfrequenzen $\omega_{\text {Mod }} \approx 1000 \mathrm{sec}^{-1}$ gegenüber $\omega_{\text {Mod }} \geq 10000 \mathrm{sec}^{-1}$ bei der $T$-Modulation und weniger an den intrinsischen Eigenschaften wie den effektiven Massen der untersuchten Fermiflächenquerschnitte (siehe Gl.3.25, Gl.3.33. [Thi92] oder [Sho84]). Die ermittelte Frequenz $F_{2}$ von $7.755 \pm 0.003 \mathrm{kT}$ (siehe Abb.3.11 entspricht einem Fermiflächenquerschnitt $A_{2}=73.99 \pm 3 \mathrm{~nm}^{-2}$. Ihr dHvASignal verschwindet bei sinkendem Magnetfeld abrupt mit einer peakförmigen Signaländerung um mehr als eine Größenordnung. Dies ist dem Verhalten bei der Detektion mit $T$ Modulation ähnlich, allerdings verschwinden die Oszillationen der Frequenz $F_{2}$ direkt mit einsetzender Supraleitung für $B \leq B_{\mathrm{c} 2}=7.7 \mathrm{~T}$ (siehe Abb.3.12. 


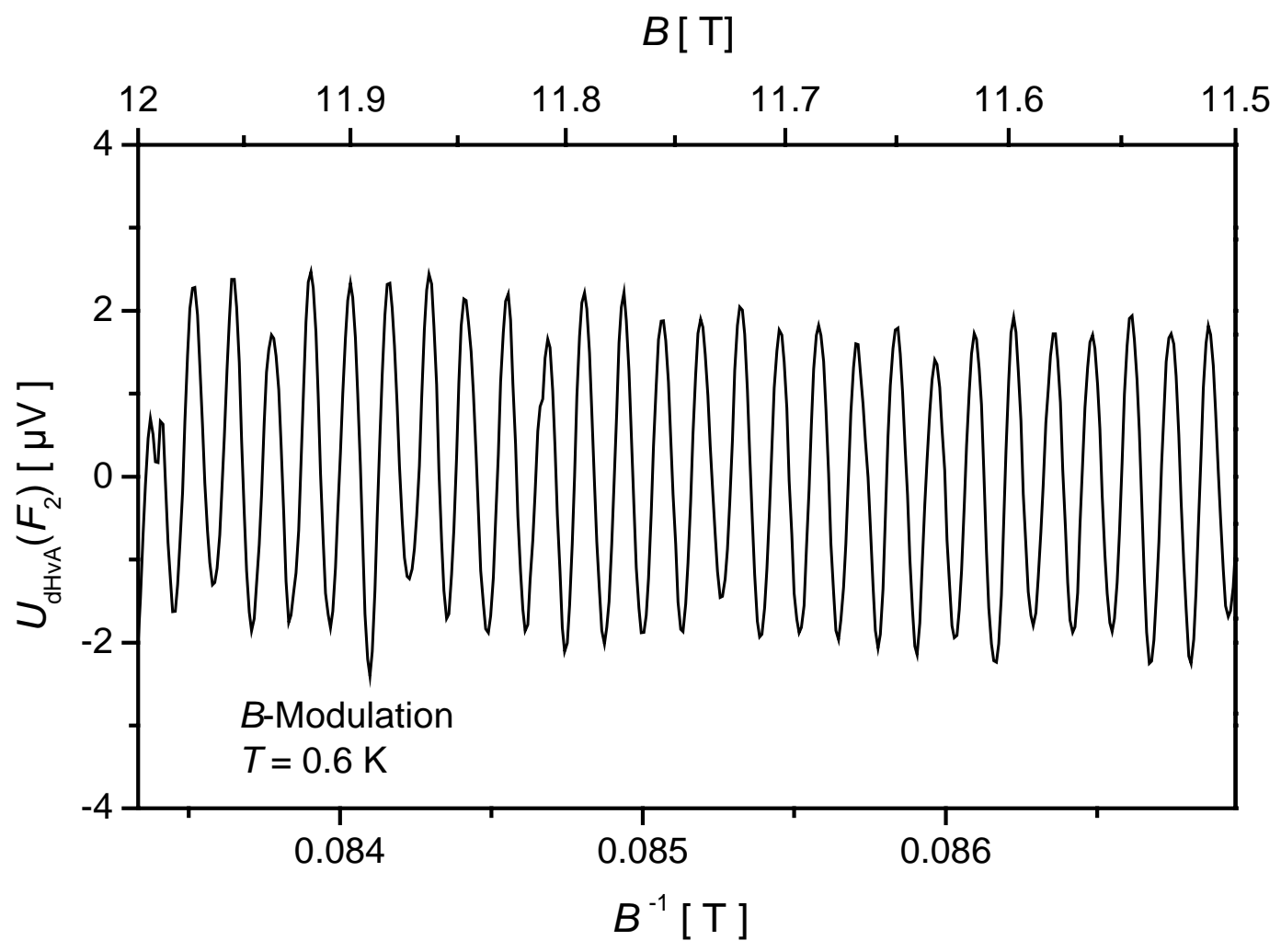

Abb.3.10 DHvA-Signal mittels B-Modulation in der Orientierung B $\|$ [001] im Feldbereich 12-11.5 T.
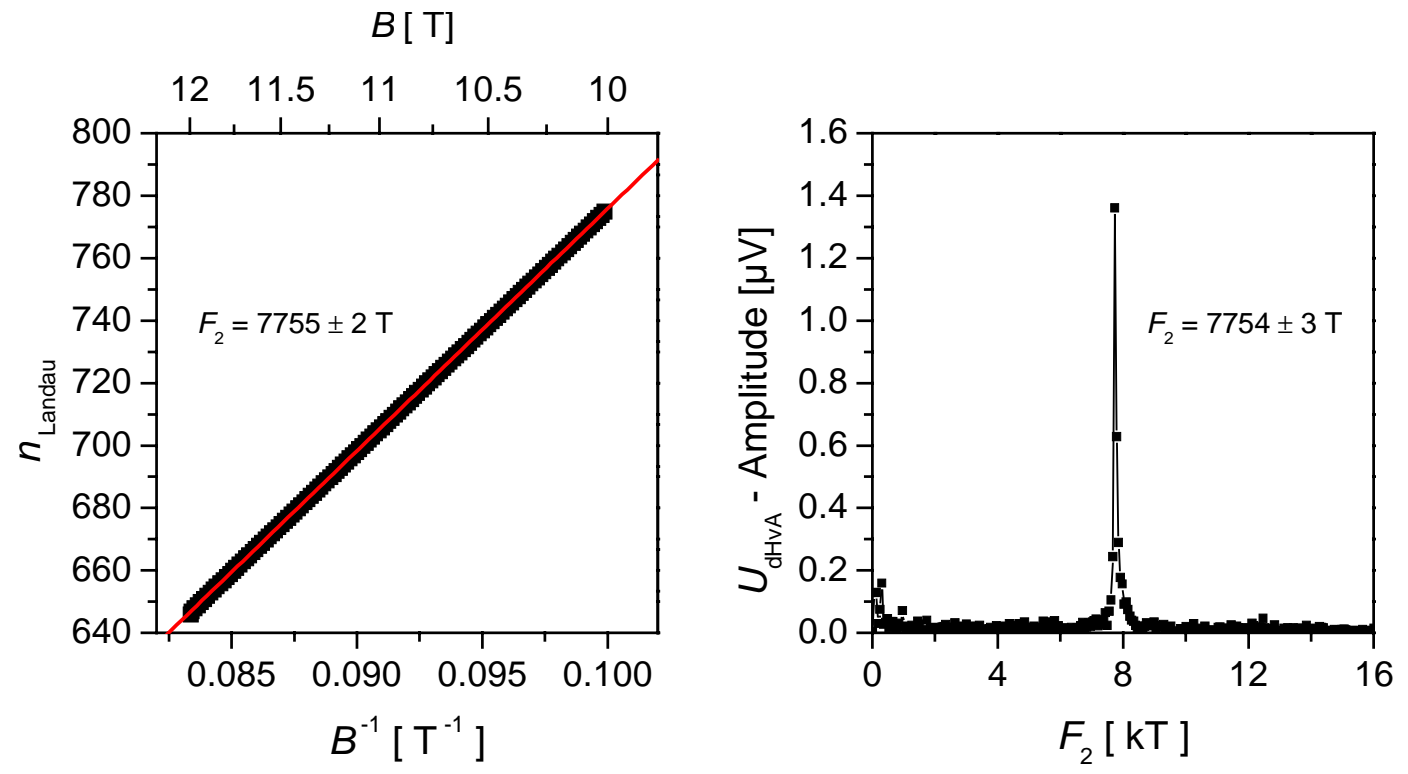

Abb.3.11 Frequenzanalysen des dHvA-Signals der Abb.3.10 im Feldbereich 12-10 T. 


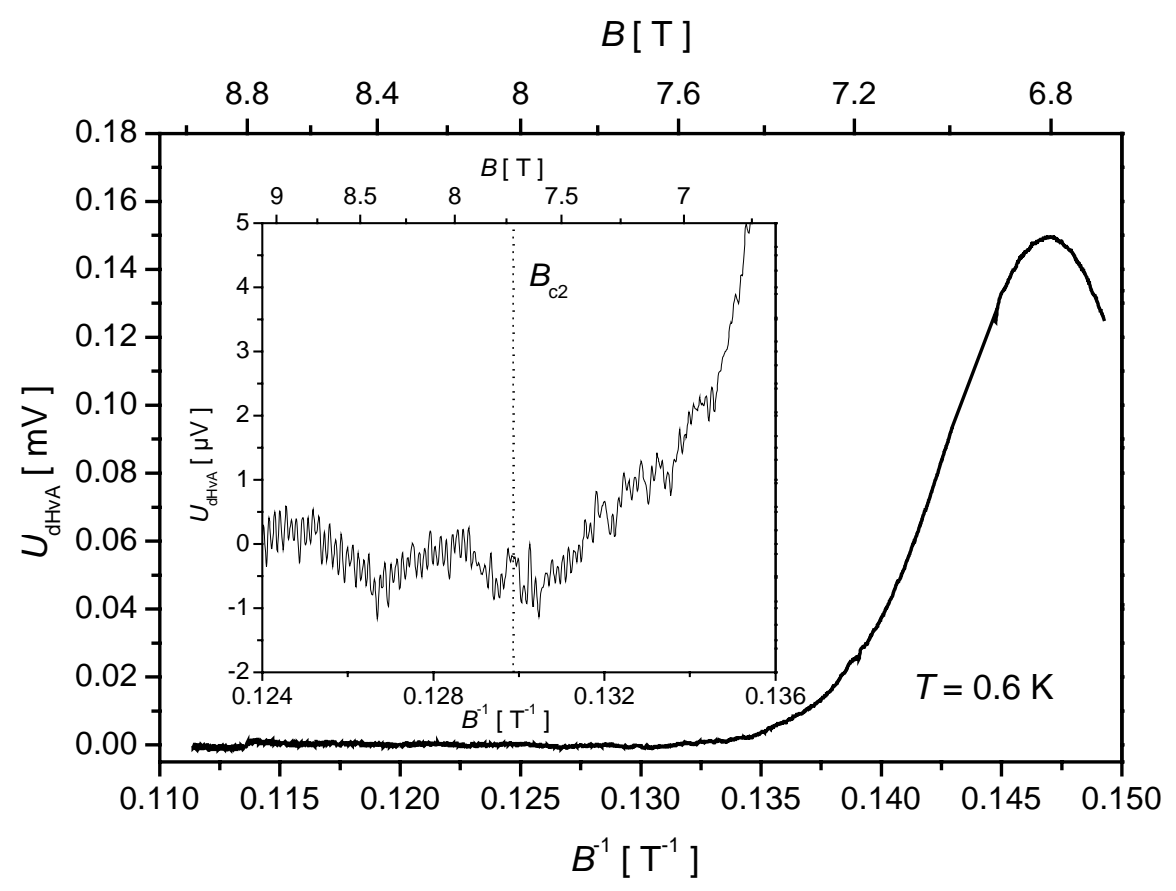

Abb.3.12 Verschwinden der dHvA-Oszillationen der Frequenz $F_{2}$ bei $B \approx B_{c 2}$ (siehe Inset) im ,peakförmigen " Signalanstieg.
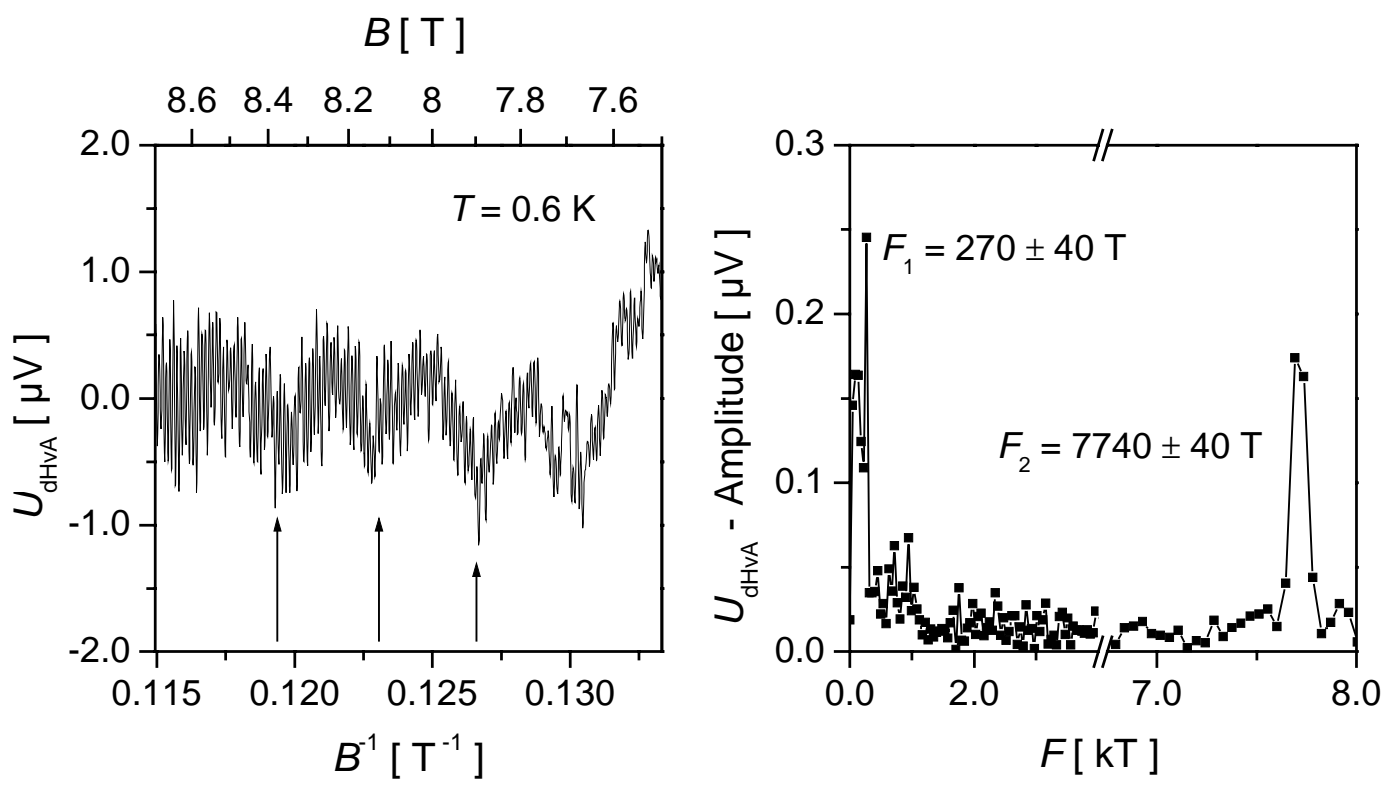

Abb.3.13 Auftreten der Frequenz $F_{1}$ als schwache Signalvariation bei Verwendung der B-Modulationstechnik. Die Pfeile der Abbildung a) markieren die ungefähre Periodizitätslänge $\Delta_{l}\left(B^{-1}\right)$ und b) zeigt das Ergebnis der Fourieranalyse für den in a) dargestellten Feldbereich. 
Die Frequenz $F_{1}$ wird nur mit kleiner Amplitude bei tiefen Feldern sichtbar. Dies ist mit der in Kap.3.3.3 beschriebenen schlechteren Meßbedingungen für kleine dHvAFrequenzen im Feldmodulationsfall zu begründen. In Abb.3.13a ist die Frequenz $F_{1} \mathrm{im}$ Meßbereich 7.6 T $<B<8$ als schwache Modulation des Signals zu beobachten, was sich auch in der für diesen Feldbereich durchgeführten Fourieranalyse niederschlägt. Aufgrund des kleinen Signals der Frequenz $F_{1}$ konnte diese mittels $B$-Modulation nicht weiter charakterisiert werden.

Die Bestimmung der effektiven Elektronenmasse für den Querschnitt $A_{2}$ erfolgte aus der Temperaturabhängigkeit des dHvA-Signals in der Orientierung $B \|$ [001]. Dazu wurden Oszillationen der Frequenz $F_{2}$ im Feldbereich $11.8 \mathrm{~T} \geq B \geq 11.3 \mathrm{~T}$ für jeweils konstante Temperaturen $1.7 \mathrm{~K} \geq T \geq 0.35 \mathrm{~K}$ detektiert.
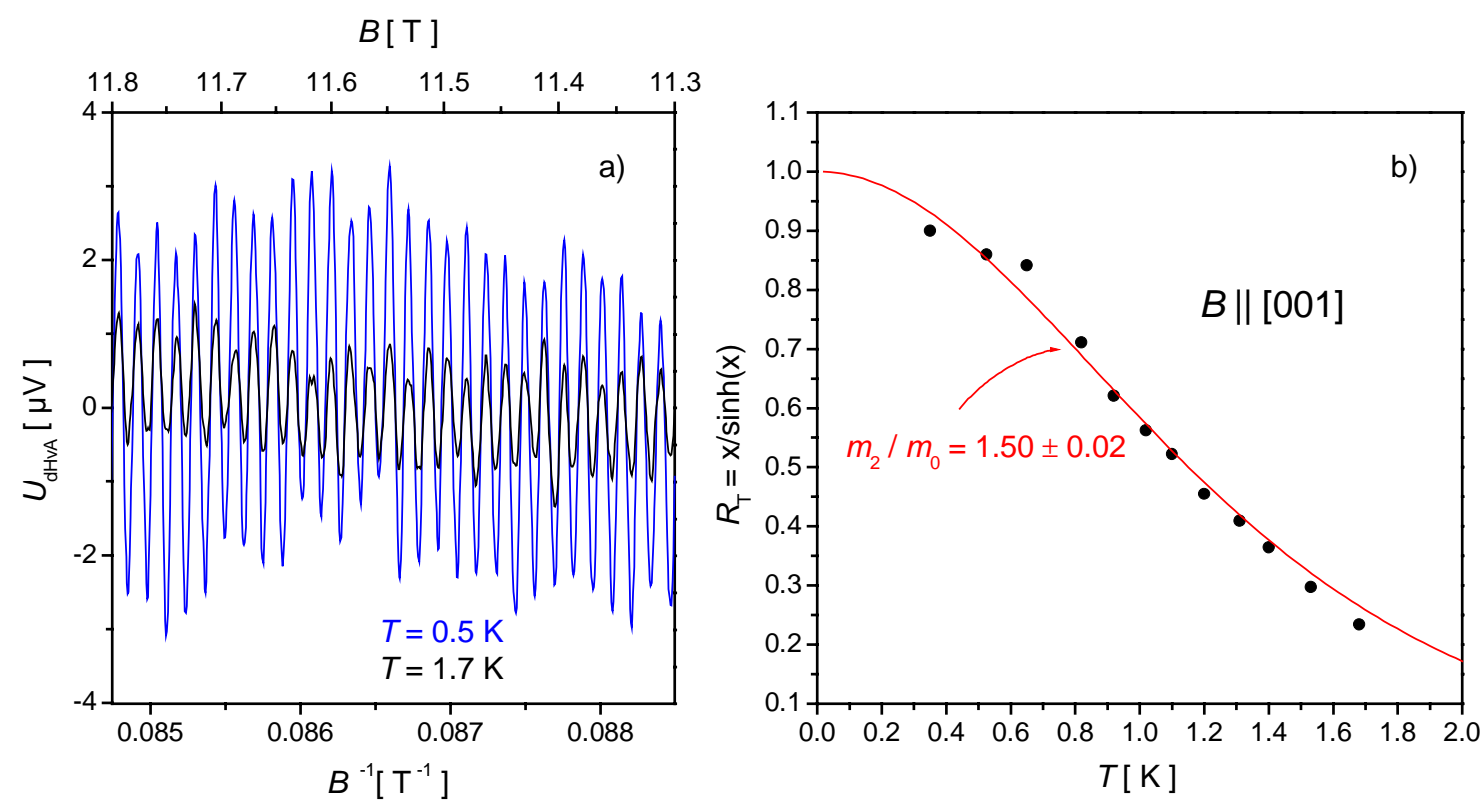

Abb.3.14 a) Temperaturabhängigkeit der dHvA-Amplitude von $F_{2}$ für zwei repräsentative Meßkurven in der Orientierung $B \|$ [001].

b) Anpassung der experimentell bestimmten Amplitudenabnahme (schwarze Symbole) gemäß Gl.3.12 mit $m_{2} / m_{0}=1.50(2)$ (rote Kurve).

Erwartungsgemäß nimmt die Amplitude mit steigender Temperatur ab, was in der Abb.3.14a exemplarisch an den Kurven für $T=0.5 \mathrm{~K}$ und $1.7 \mathrm{~K}$ gezeigt ist. Die Anpassung der für diskrete Meßtemperaturen ermittelten Amplituden gemäß Gl.3.12 ergibt die effektive Elektronenmasse $m_{2}=1.50(2) \cdot m_{0}$ im Fermiflächenquerschnitt $A_{2}$ (siehe Abb.3.14p). 
Damit ergibt sich für die Fermigeschwindigkeit im zugehörigen Fermiflächenquerschnitt $v_{\mathrm{F} 2}=\sqrt{2 e \hbar F_{2}} / m_{2}=3.75(5) \cdot 10^{5} \mathrm{~m} / \mathrm{sec}$.

Mit Kenntnis der effektiven Masse ist eine Beschreibung der Feldabhängigkeit der Amplitude gemäß Gl.3.19 und Gl.3.26 möglich. Ähnlich dem Vorgehen bei T-Modulation ergibt demnach die Normierung der feldabhängigen Oszillationsamplitude auf $R_{\mathrm{T}} \cdot(B)^{(1 / 2)} \cdot J_{2}(\mathrm{y})$ den Dinglefaktor $R_{\mathrm{D}}$ zuzüglich einer Konstanten. Die lineare Anpassung des experimentellen Verlaufs von $\ln \left(R_{\mathrm{D}}\right)$ in Abb.3.15 ergibt eine Steigung von $-66 \pm 2 \mathrm{~T}$. Mit der effektiven Elektronenmasse $m_{2}=1.5 \cdot m_{0}$ gilt demnach für die Dingletemperatur $T_{\mathrm{D}}=3.0 \pm 0.1 \mathrm{~K}$ und die Streuzeit der Elektronen beträgt $\tau_{0}=(0.4 \pm 0.01) \cdot 10^{-12} \mathrm{sec}$

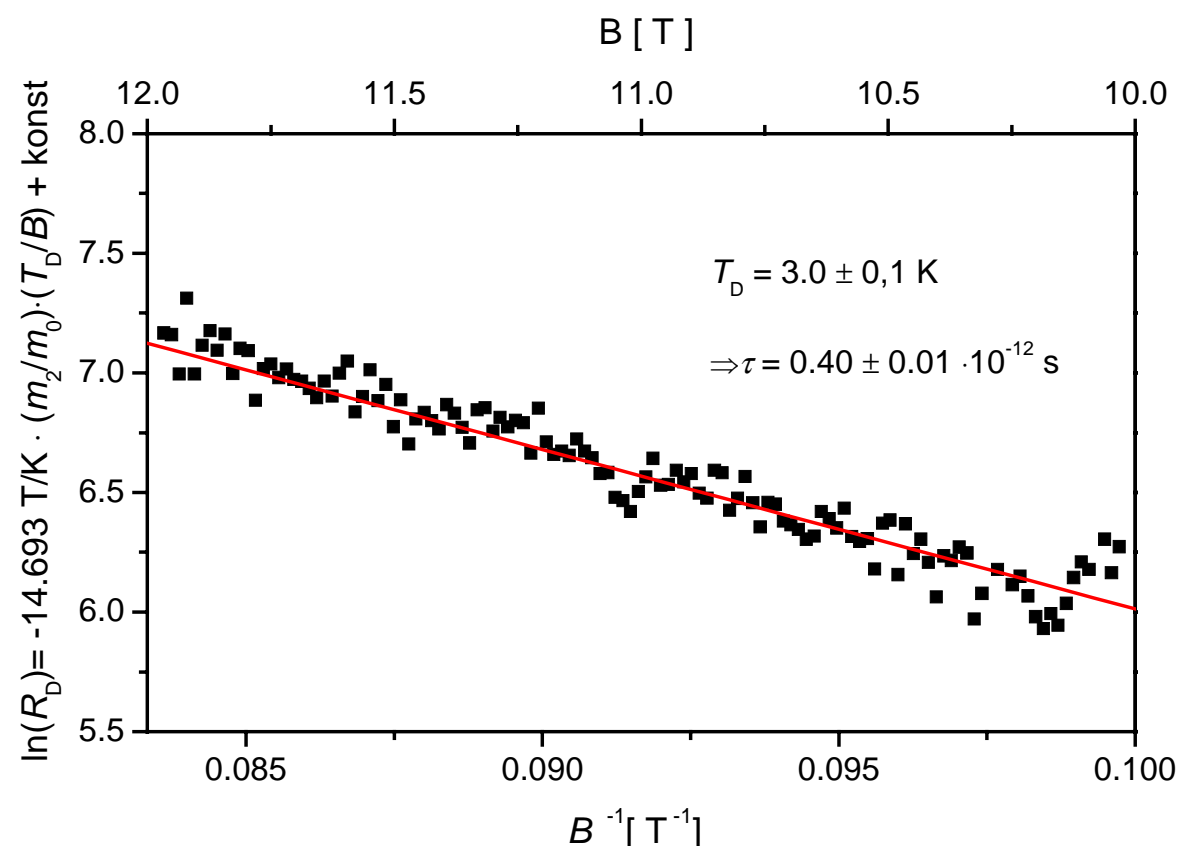

Abb.3.15 Bestimmung der Dingletemperatur $T_{\mathrm{D}}$ aus der Feldabhängikeit von $\ln \left(R_{\mathrm{D}}\right)$. Die schwarzen Symbole ergeben sich aus der Auswertung der Oszillationsamplitude einer Messung zwischen 12 und 10 T gemäß Gl.3.19 und Gl.3.26. Die rote Kurve entspricht der linearen Anpassung an die experimentellen Werte.

Die Charakterisierung der Winkelabhängigkeit des Fermiflächenquerschnittes $A_{2}$ erfolgte im Winkelbereich $\varphi \leq 20^{\circ}$ für Verkippungen des Magnetfeldes wiederum in der $(a, c)$ Ebene. Für größere $\varphi$ konnten keine dHvA-Oszillationen mehr detektiert werden. Man erhält die in Abb.3.16 dargestellte schwache Abnahme des Fermiflächenquerschnittes $A_{2}(\varphi=0) / A_{2}(\varphi=20) \approx 74 / 72.5$ um maximal $2 \%$. Zum besseren Vergleich mit dem im Y- 

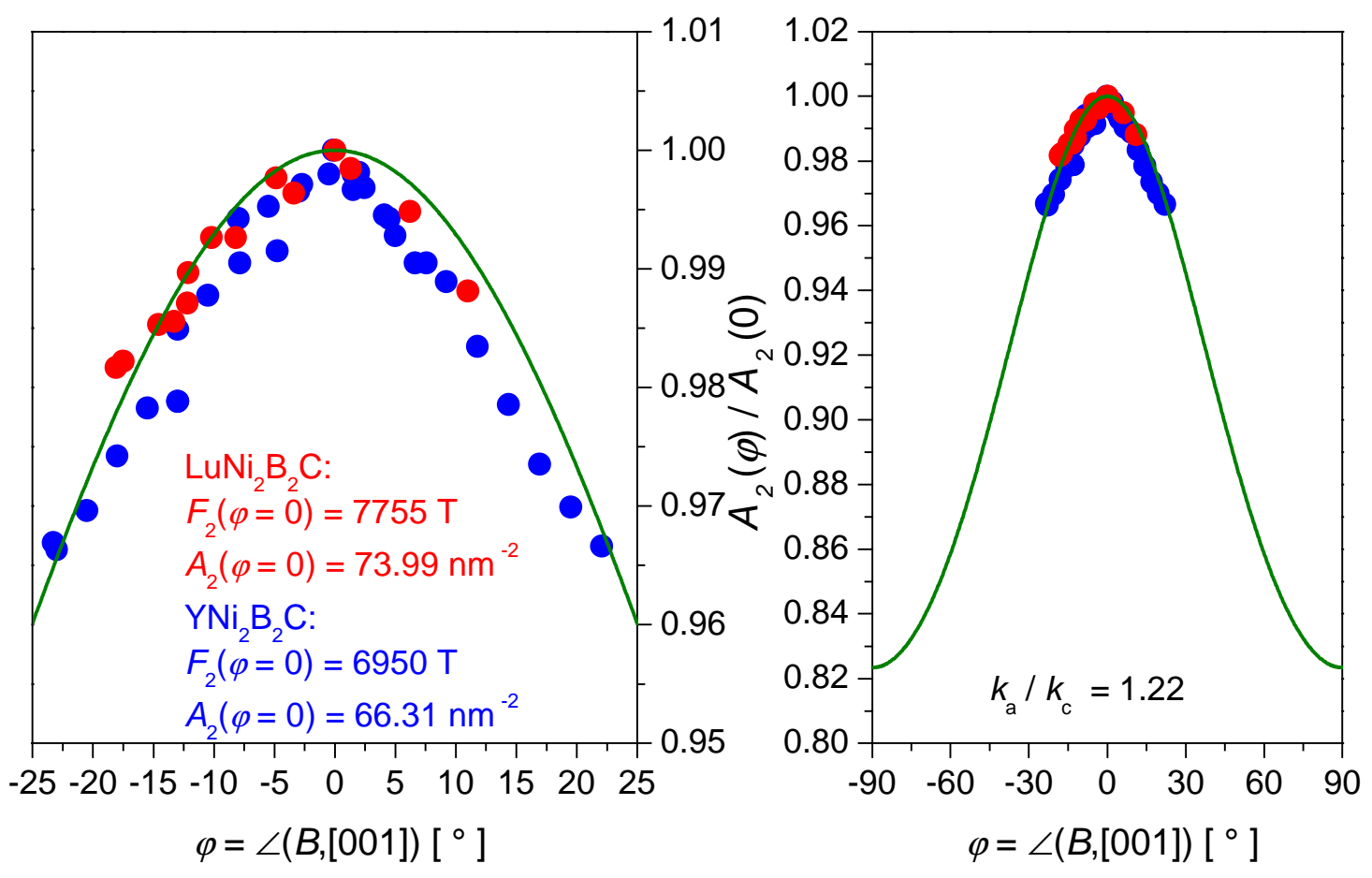

Abb.3.16 Winkelabhängigkeit der Frequenz $F_{2}$ bei Verkippung des Magnetfeldes in der (a,c)-Ebene. Die Winkelabhängigkeit im Lu-System (rote Symbole) ist zum besseren Vergleich mit der im YSystem (blaue Symbole nach [Hei95]) gemeinsam dargestellt.

System beobachten Querschnitt $A_{2}(\varphi=0)=63.31 \mathrm{~nm}^{-2}$ ist die dort beobachtete Winkelabhängigkeit [Hei95] mit aufgeführt. Gemäß Abb.3.16 ist die schwache Anisotropie für $F_{2}$ in beiden Systemen nahezu gleich. In Abb.3.16 ist als grüne Kurve wiederum die zu erwartende Winkelabhängigkeit für eine rotationsellipsoide Fermifläche mit eingezeichnet. Der Verlauf ist die Anpassung gemäß Gl.3.35, allerdings hier mit langer Achse in der $(a, b)$-Ebene und $k_{\mathrm{a}} / k_{\mathrm{c}}=1.22$. Der aus dieser Anpassung für die Symmetrielage $B \|$ [100] ermittelte mögliche Fermiflächenquerschnitt von $54.97 \mathrm{~nm}^{-2}$ kann als Indiz für eine Zuordnung der gemessenen Fläche $A_{2}$ zu Fermiflächenquerschnitten aus Bandstrukturrechnungen dienen. Bei Gültigkeit der aus Abb.3.16 folgenden Äquivalenz der Frequenzen $F_{2}$ im $\mathrm{Y}$ - und Lu-System erscheint aber wahrscheinlicher, daß für Winkel $\varphi>30^{\circ}$ die Geschlossenheit der Flächenberandung nicht mehr erfüllt ist. Auch in $\mathrm{YNi}_{2} \mathrm{~B}_{2} \mathrm{C}$ verschwinden die dHvA-Oszillationen in diesem Winkelbereich [Ngu96], obwohl bei sehr viel höheren Magnetfeldern $B \leq 23 \mathrm{~T}$ und einer von der plättchenhaften Geometrie der Proben weniger beeinflußten Cantilevermethode gemessen wurde. 
Mit den hier angewandten Magnetfeldern $B<12 \mathrm{~T}$ und Probenqualitäten $T_{\mathrm{D}} \approx 3 \mathrm{~K}$ konnten neben $F_{2}$ keine neuen Frequenzen beobachtet werden.

\subsubsection{Suszeptibilitätsmessung en für $B \|$ [001]}

Um die in den dHvA-Experimenten auftretende peakförmige Signaländerung im supraleitenden Zustand etwas genauer zu charakterisieren, wurden zusätzliche Suszeptibiltätsmessungen für verschieden Temperaturen $T \leq T_{\mathrm{c}}$ durchgeführt. Abb.3.17 zeigt Messungen des Betrages der Suszeptibilität $\chi_{\mathrm{ac}}$, wobei das gemäß Gl.3.2 gemessene Spannungssignal bei $B=0 \mathrm{~T}$ auf einen perfekten Diamagneten mit $\chi_{\mathrm{ac}}=-1$ normiert ist. Für alle Temperaturen sieht man schon für kleinste Magnetfelder einen Anstieg der Suszeptibilität $\chi_{\mathrm{ac}}>-1$. Dies ist im Einklang mit dem berechneten $B_{\mathrm{cl}}(0 \mathrm{~K}) \approx 30 \mathrm{mT}$ aus Ergebnissen von spezifischen Wärmemessungen am TypII-Supraleiter $\mathrm{LuNi}_{2} \mathrm{~B}_{2} \mathrm{C}$ ([Hil99]). Zusätzlich zur Signalvariation bei kleinen Feldern ist bis zu $T=13.0 \mathrm{~K}$ ein feldabhängiges peakförmiges diamagnetisches Signal zu beobachten, daß mit steigender Temperatur flacher und schärfer wird. Das diamagnetische Signal im Supraleiter ist direkt proportional zu dem es erzeugenden supraleitenden Abschirmstrom $j_{c}$, für den Eskildsen et al. [Esk97] bei vergleichbaren Feldern nahe $B_{\mathrm{c} 2}$ einen peakförmigen Anstieg sowohl für $\mathrm{LuNi}_{2} \mathrm{~B}_{2} \mathrm{C}$ als auch $\mathrm{YNi}_{2} \mathrm{~B}_{2} \mathrm{C}$ beobachten. Im Vergleich mit Ergebnissen aus der Kleinwinkelneutronenstreuung diskutieren sie das Maximum in $j_{\mathrm{c}}$ im Rahmen des kollektiven Pinningmodells nach Larkin und Ovchinnikov [Lar79]. Demnach liegt für $\mathrm{YNi}_{2} \mathrm{~B}_{2} \mathrm{C}$ und $\mathrm{LuNi}_{2} \mathrm{~B}_{2} \mathrm{C}$ für Felder $B \leq B_{\mathrm{c} 2}$ ein Flußliniengitter mit unnormal kleiner Schersteiffigkeit vor, welches sich an statistisch verteilte Pinningzentren anlagert und für $B_{\text {Peak }}$ eine Überhöhung des kritischen Stromes bzw. der diamagnetischen Suszeptibilität bewirkt. Diese Erklärung des Effektes untermauert die von Heinecke [Hei95] angestellte Vermutung, daß die in $\mathrm{YNi}_{2} \mathrm{~B}_{2} \mathrm{C}$ für einen eingeschränkten Feldbereich beobachtete Dämpfung der dHvA-Oszillationen für $F_{1}$ kein Effekt einer sich unterhalb $B_{\mathrm{c} 2}$ ausbildenden Energielücke sind, sondern durch gestörtes Eindringen des magnetischen Feldes durch überhöhte Pinnkraft bewirkt wird. 

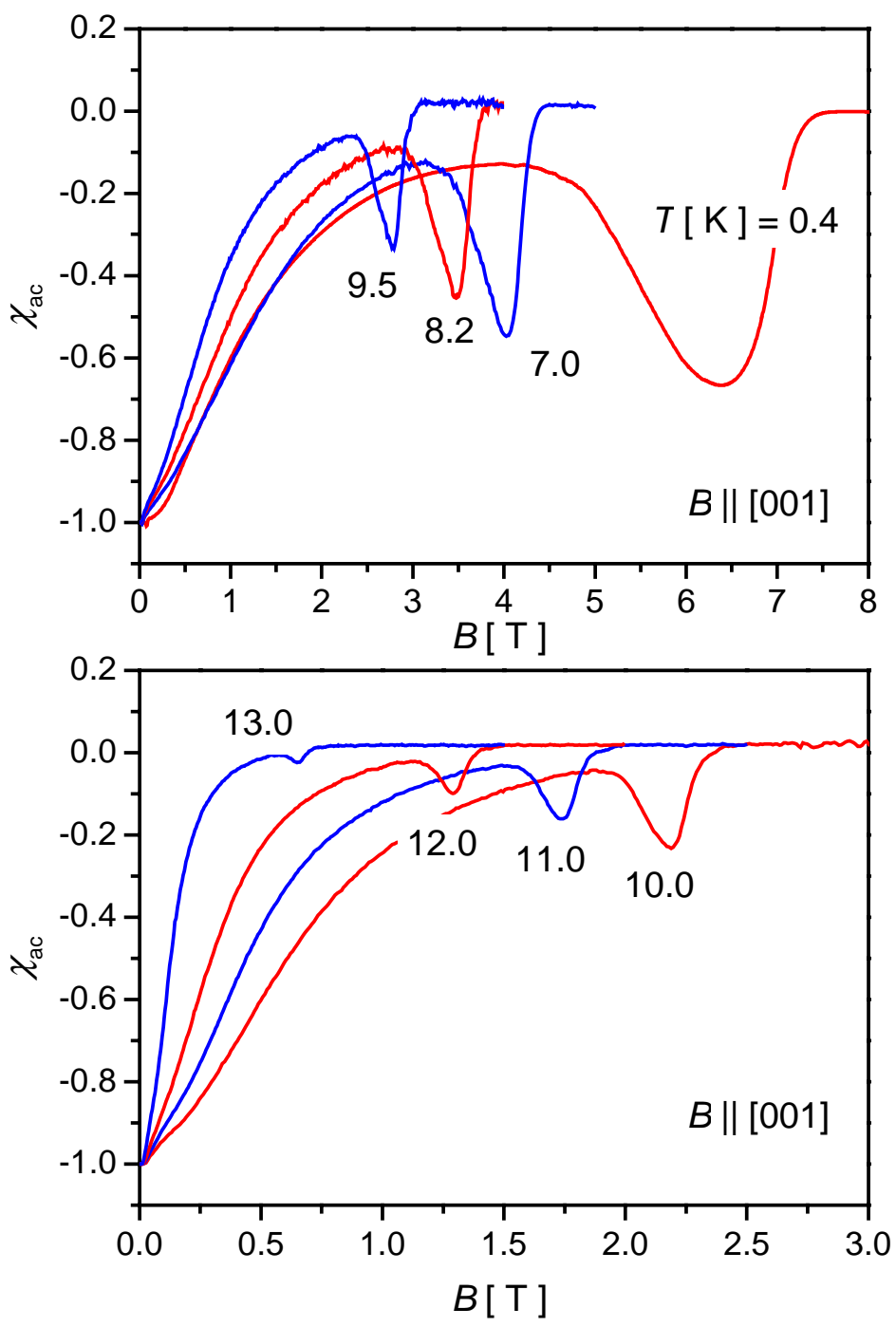

Abb.3.17 Messungen der Wechselfeldsuszeptibilität $\chi_{\mathrm{ac}}(B)$ bei verschiedenen Temperaturen mit „Peakeffekt“ für Felder $B<B_{\mathrm{c} 2}(T)$ in der Orientierung $B \|[001]$.

Fraglich bleibt aber weiterhin, was in hochwertigen Einkristallen $\left(T_{\mathrm{D}} \approx 3 \mathrm{~K}, R R R \geq 30\right)$ als Pinningzentren wirken kann. Eine ähnliche Diskrepanz in der Beschreibung des Peakeffektes im kollektiven Pinningmodell für reine Einkristalle sehen auch Modler et al. [Mod96].

Die nach obiger Argumentation vermutlich mit einem Ordungsübergang im Flußliniengitter verbundene Änderung der Suszeptibiltät bestimmt das Meßsignal unterhalb 6.5 T der dHvA-Messungen in Abb.3.4 Die Bestimmung einer zusätzlichen Streurate $\left(1 / \tau_{\mathrm{sl}}\right) \mathrm{im}$ supraleitenden Zustand aus den Meßdaten der Abb.3.6 erscheint daher nicht sinnvoll zu sein. 


\subsection{Widerstandsmes sungen}

In diesem Abschnitt werden die zur Charakterisierung der Anisotropie von $B_{c 2}$ in der $(a, b)$-Ebene und die für feste Orientierung $B \|[001]$ durchgeführten Widerstandsmessungen präsentiert.

Die Widerstandsmessungen mit $B$ innerhalb der $(a, b)$-Ebene des $\mathrm{LuNi}_{2} \mathrm{~B}_{2} \mathrm{C}$-EK dienen der Überprüfung der Tieftemperaturvorhersagen für $B_{\mathrm{c} 2}(T, \alpha)$ aus dem Modell der unkonventionellen $d$-Wellen-Supraleitung nach [Wan98], wobei $\alpha$ den Winkel des angelegten Magnetfeldes zur [100] - Richtung des Kristalls beschreibt. Die dort ermittelte $B_{\mathrm{c} 2}$-Anisotropie wird ausgedrückt durch :

Gl.3.36 $\quad B_{\mathrm{c} 2}(T, \alpha)=0.5 \cdot\left[B_{\mathrm{c} 2}(100)+B_{\mathrm{c} 2}(110)\right] \cdot[1+\Gamma \cos (4 \alpha)]$

Dabei ist $\Gamma$ eine Art differenzielle Anisotropie $\Gamma(T)=\frac{B_{c 2}(100)-B_{c 2}(110)}{B_{c 2}(100)+B_{c 2}(110)}(T)$.

Eine weitere Aussage dieser Beschreibung ist die temperaturunabhängige Konstanz der relativen Anisotropie $\Gamma_{\mathrm{in}}=B_{\mathrm{c} 2}(100)(T) / B_{\mathrm{c} 2}(110)(T)$.

Die in [Wan98] implizit gegebenen Tieftemperaturvorhersagen für $\Gamma$ und $\Gamma_{\text {in }}$ sollen durch experimentell bestimmte Werte unterhalb $4.2 \mathrm{~K}$ überprüft werden. Zusätzlich zu diesen Widerstandsexperimenten mit Magnetfeldern innerhalb der $(a, b)$-Ebene des Systems wurden eben solche mit fester Orientierung $B \|[001]$ zur Ermittlung des $B_{\mathrm{c} 2}(T)$-Verlaufs speziell nahe $T_{\mathrm{c}}=16.3 \mathrm{~K}$ durchgeführt.

Die für Temperaturen $T \leq T_{\mathrm{c}}$ in der Orientierung $B \|$ [001] durchgeführten feldabhängigen Widerstandsmessungen dienen zur Bestimmung des $B_{\mathrm{c} 2}(T)$-Verlaufes.

\subsubsection{Ergebnisse}

\subsubsection{Widerstandsmessungen für $B \|(a, b)$-Ebene}

Abb.3.18 zeigt die bei der Meßtemperatur $T=0.4 \mathrm{~K}$ aufgenommenen Kurven des spezifischen Widerstandes $\rho(B)$ als Funktion des Feldes. Dargestellt sind Messungen für Verkippungswinkel $\alpha \angle(B,[100])<45^{\circ}$ zwischen der Magnetfeldrichtung und der [100]Kristallachse. Man sieht einen kontinuierlichen Anstieg des Übergangsfeldes zur Normalleitung für sinkende Winkel $\alpha$. 

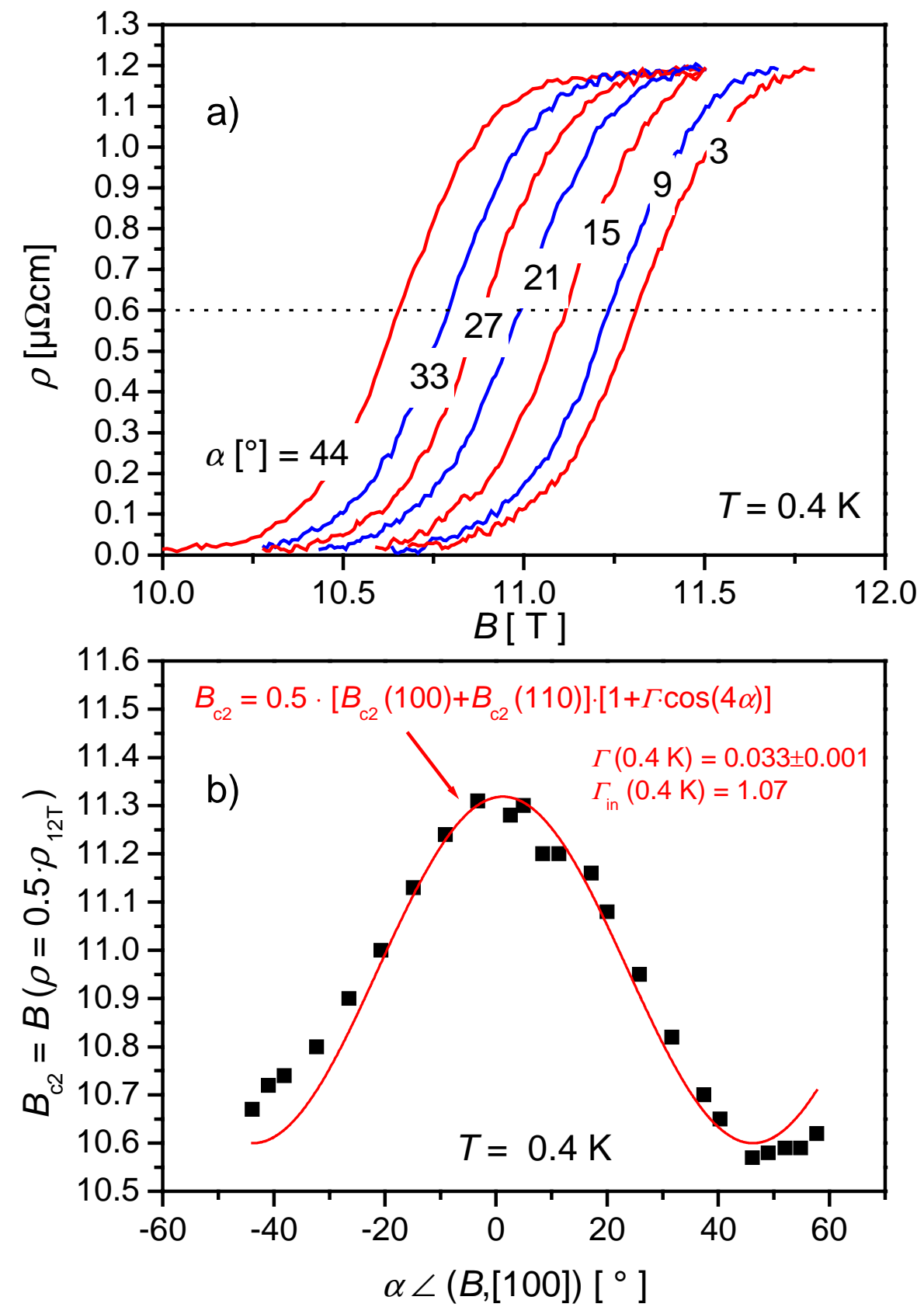

Abb.3.18 a) Messungen des spezifischen Widerstandes bei tiefstmöglicher Temperatur $T=0.4 \mathrm{~K}$ im Winkelbereich $\alpha \angle(B,[100])<45^{\circ}$.

b) Aus a) bestimmter $B_{\mathrm{c} 2}$-Verlauf mit $\rho\left(B_{\mathrm{c} 2}\right)=0.5 \cdot \rho(B=12 \mathrm{~T})$. Rot dargestellt die Anpassung nach Gl.3.36. 

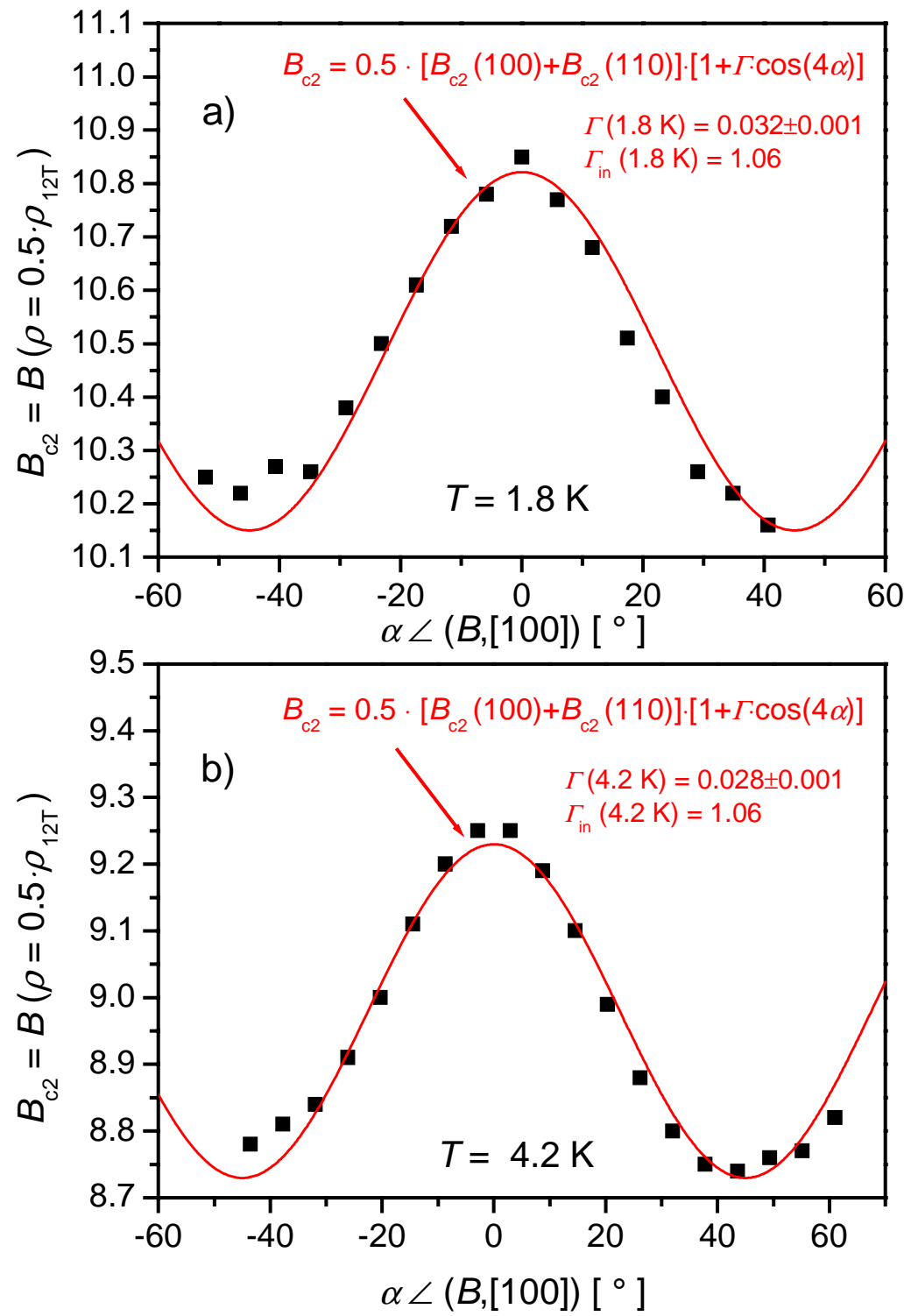

Abb.3.19 Aus Widerstandsmessungen bei $T=1.8 \mathrm{~K}$ (a) und $T=4.2 \mathrm{~K}$ (b) bestimmter $B_{\mathrm{c} 2}$-Verlauf mit $\rho\left(B_{\mathrm{c} 2}\right)=0.5 \cdot \rho(B=12 \mathrm{~T})$. Rot dargestell die Anpassungen nach Gl.3.36.

Wertet man $B_{\mathrm{c} 2}$ aus dem halben Übergangswert $\rho\left(B_{\mathrm{c} 2}\right)=0.5 \cdot \rho(B=12 \mathrm{~T})$ aus, der in Abb.3.18a durch die gestrichelte Linie markiert ist, so erhält man den als schwarze Quadrate in Abb.3.18b dargestellten Verlauf $B_{\mathrm{c} 2}(\alpha)$. Dieser läßt sich gut mit der Winkelabhängigkeit nach Gl.3.36 beschreiben, die als rote Kurve angepaßt wurde. Sie liefert für $\Gamma(0.4 \mathrm{~K})=0.033 \pm 0.001$ und $\Gamma_{\text {in }}(0.4 \mathrm{~K})=1.07$. Entsprechende Messungen bei $T=1.8 \mathrm{~K}$ und $T=4.2 \mathrm{~K}$ ergeben die in Abb.3.19 dargestellten $B_{\mathrm{c} 2}(\alpha)$-Verläufe. Die Anisotropieparameter aus den Anpassungen gemäß Gl.3.36 ergeben sich zu $\Gamma(1.8 \mathrm{~K})=0.032 \pm 0.001$ 
und $\Gamma_{\text {in }}(1.8 \mathrm{~K})=1.06$ bzw. $\Gamma(4.2 \mathrm{~K})=0.028 \pm 0.001$ und $\Gamma_{\text {in }}(4.2 \mathrm{~K})=1.06$. Abb.3.20 vergleicht diese Werte für $\Gamma(T)$ mit der theoretischen Vorhersage nach [Wan98] in einer Auftragung gegen die relative Temperatur $t=1-T / T_{\mathrm{c}}$. Zusätzlich zu den hier für Temperaturen unterhalb $T=4.2 \mathrm{~K}$ bestimmten Werten sind die aus Magnetisierungsmessungen von Metlushko et al. [Met97] bestimmten mit eingezeichnet. Diese Daten lassen sich an die hier aus Widerstandsmessungen gewonnenen gut anschließen. Während die experimentellen Werte unterhalb etwa $8 \mathrm{~K}(t \geq 0.5)$ nahezu sättigen, steigt der theoretische Verlauf für sinkende Temperaturen kontinuierlich an. Die Differenz zwischen Theorie und Experiment beträgt daher bei tiefster Temperatur mehr als $75 \%$ des theoretischen Wertes.

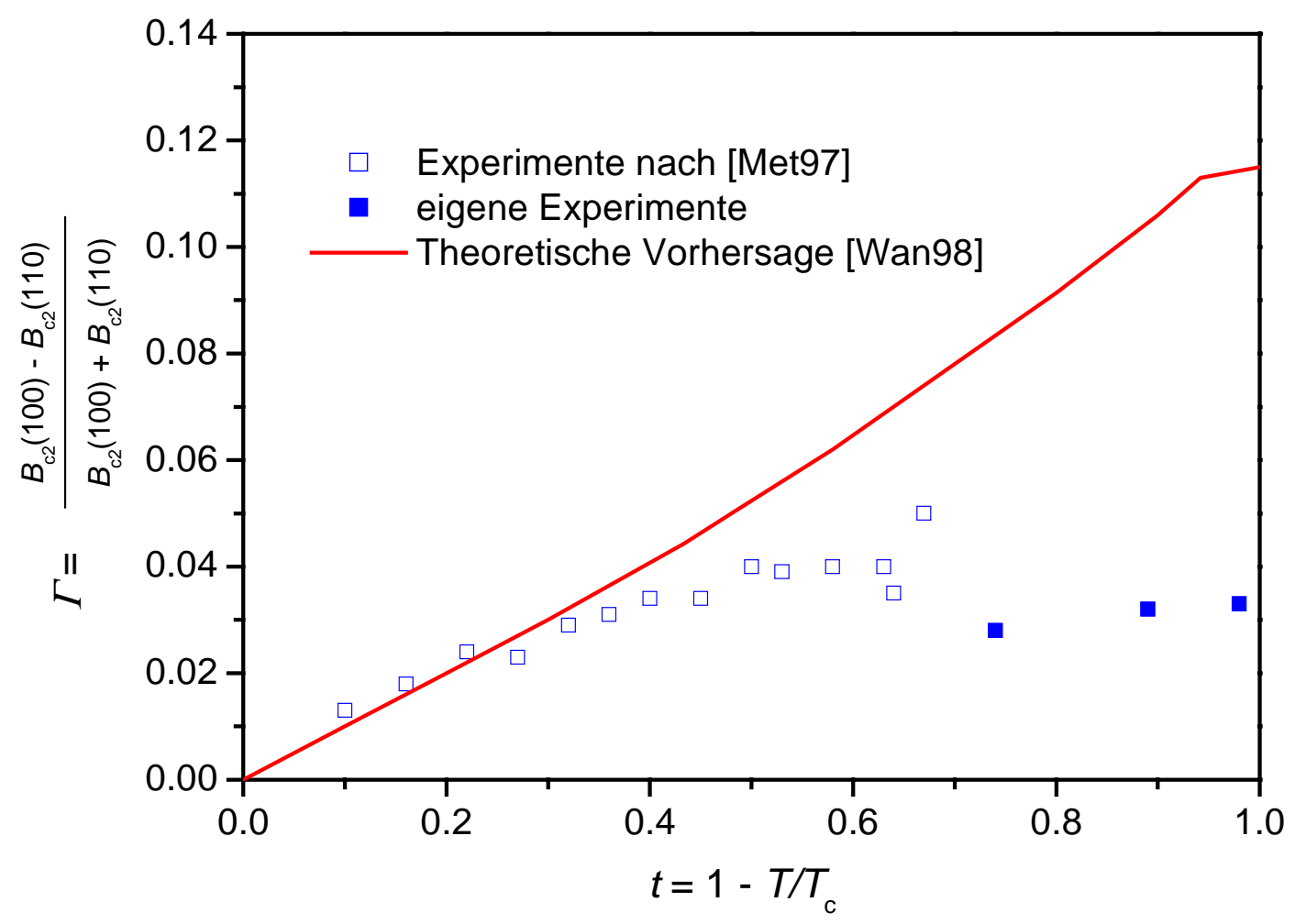

Abb.3.20 Vergleich der experimentell gewonnen relativen Anisotropie $\Gamma$ (quadratische Symbole) mit der theoretischen Vorhersage nach [Wan98]. Offene Symbole nach [Met97].

Die hier bei tiefster Temperatur am hochwertigen Einkristall im clean limit $l * / \xi_{\mathrm{Gl}}=\left[\left(v_{\mathrm{F}} \tau\right) /\left(\Phi_{0} / 2 \pi B_{\mathrm{c} 2}\right)^{1 / 2}\right] \approx 23$ durchgeführten Widerstandsexperimente widersprechen also deutlich den aus dem $d$-Wellen-Modell bestimmten Aussagen zur Tempera- 
turabhängigkeit der relativen Anisotropie $\Gamma$. Lediglich die Konstanz von $\Gamma_{\text {in }} \approx 1.06$ ist im Einklang mit der dort gemachten Vorhersage.

\subsubsection{Widerstandsmessungen für $B \|(c)$}

Abb.3.21 zeigt durchgeführte Widerstandsmessungen in der Orientierung $B \|[001]$. Ähnlich zum Vorgehen für $B \|(a, b)$-Ebene wurde das kritische Feld aus dem halben Übergangswert bestimmt, d.h. $\rho\left(B_{\mathrm{c} 2}\right)=0.5 \cdot \rho(12 \mathrm{~T})$. Der daraus bestimmte $B_{\mathrm{c} 2}(T)$-Verlauf ist in Abb.3.21b dargestellt. Die Kurve zeigt im ganzen Temperaturbereich eine deutliche Abweichung vom für viele Supraleiter beobachteten parabelförmigen Verlauf:

Gl. $3.37 \quad B_{\mathrm{c} 2}(T)=B_{\mathrm{c} 2}(0 \mathrm{~K}) \cdot\left(1-\frac{T^{2}}{T_{\mathrm{c}}^{2}}\right)$

Speziell im Temperaturbereich nahe $T_{\mathrm{c}}$ für $T \geq 12 \mathrm{~K}$ ist eine deutliche positive Krümmung der experimentellen Kurve zu beobachten. Diese positive Krümmung in der Temperaturabhängigkeit des oberen kritischen Feldes tritt in gleicher Form in dem von Heinecke [Hei95] in dHvA-Experimenten untersuchten hochwertigen $\mathrm{YNi}_{2} \mathrm{~B}_{2} \mathrm{C}$-EK auf [Shu98] und wurde in schwächerem Ausmaß in qualitativ schlechteren Proben und Polykristallen beobachtet [Dre99]. 

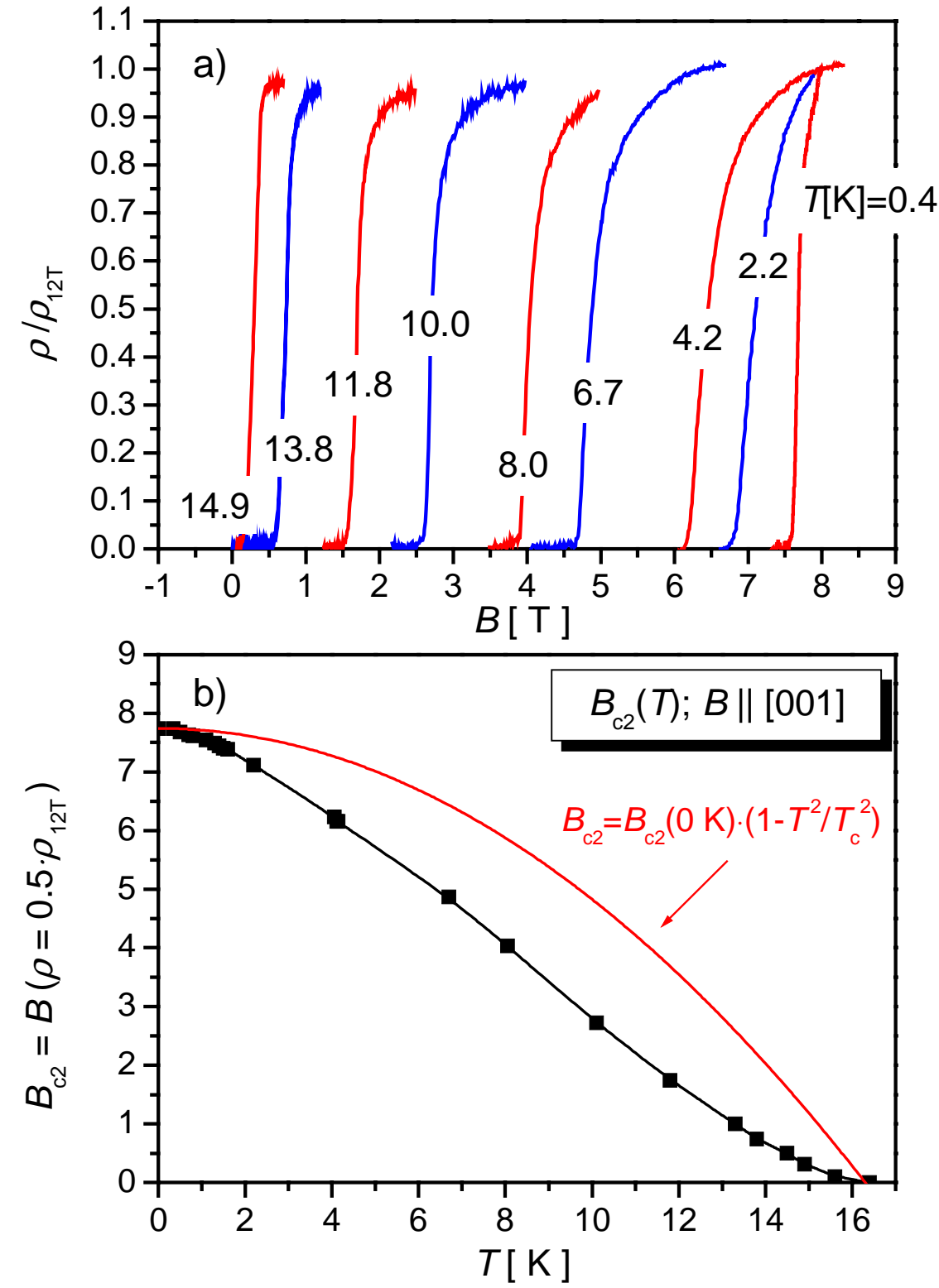

Abb.3.21 a) Magnetfeldabhängige Widerstandsmessungen eines $\mathrm{LuNi}_{2} \mathrm{~B}_{2} \mathrm{C}$-EK in der Orientierung $B$ || [001].

b) Temperaturabhängigkeit $B_{\mathrm{c} 2}(T)$ aus Messungen gemäß Abb.3.21ф im Vergleich zum Verlauf nach der erweiterten Ginzburg Landau Theorie für TypII-Supraleiter [Par69]. 


\subsection{Diskussion}

Die dHvA-Ergebnisse mit den gezeigten Winkelabhängigkeiten der Fermiflächenquerschnitte $A_{1}$ und $A_{2}$ bestätigen die aus Bandstrukturrechnungen (u.a. [Mat94], [Kim95], [Sin96], [Dre99], [Ros00]) folgende Ähnlichkeit der Fermiflächen in $\mathrm{LuNi}_{2} \mathrm{~B}_{2} \mathrm{C}$ und $\mathrm{YNi}_{2} \mathrm{~B}_{2} \mathrm{C}$. Speziell die für $\mathrm{YNi}_{2} \mathrm{~B}_{2} \mathrm{C}$ an zwei Frequenzen beobachteten dHvAOszillationen im supraleitenden Zustand konnten in $\mathrm{LuNi}_{2} \mathrm{~B}_{2} \mathrm{C}$ für eine Frequenz erstmals erfolgreich nachgewiesen werden. Die Beobachtung von dHvA-Oszillationen der Frequenz $F_{1}=278 \mathrm{~T}$ im supraleitenden Zustand bestätigt die Existenz einer teilweise verschwindendenden Energielücke $\Delta(\vec{k})$ in $\operatorname{LuNi}_{2} \mathrm{~B}_{2} \mathrm{C}$. Die Winkelabhängigkeit $A_{1}(\varphi)$ des zugehörigen Fermiflächenquerschnittes ist mit der von Nguyen [Ngu96] an $\mathrm{YNi}_{2} \mathrm{~B}_{2} \mathrm{C}$ gefundenen nahezu identisch. Allerdings können die Oszillationen dort bis zu sehr viel niedrigeren Feldern $B_{\min } \approx 2 \mathrm{~T}$ [Hei95] detektiert werden gegenüber dem hier beobachteten $B_{\min } \approx 6.5 \mathrm{~T}$. Die Gründe hierfür liegen in der unterschiedlichen Feldabhängigkeit der in Kap 3.3 besprochenen Dämpfungsfaktoren der beiden Systeme. Aus den Definitionen für $R_{\mathrm{T}} \cdot R_{\mathrm{D}} \cdot\left[x / \tanh (x)\right.$-1] in Gl.3.12 Gl.3.13 und Gl.3.34 folgt, daß $M_{0}$ empfindlich von den systemspezifischen effektiven Massen $m$ und Dingletemperaturen $T_{\mathrm{D}}$ im untersuchten Fermiflächenquerschnitt abhängt. Abb.3.22 zeigt die Feldabhängigkeit des Verhältnisses der aus den Vorfaktoren berechneten Amplituden für das Lu- und das Y-System mit $m_{1}(\mathrm{Lu})=0.5 \cdot m_{0}[\mathrm{Tok} 95]$ und $m_{1}(\mathrm{Y})=0.34 \cdot m_{0}$ [Hei95]. Ansonsten gelten gleiche Meßbedingungen $\langle T\rangle=0.4 \mathrm{~K}$ und die Dämpfungsfaktoren sind für das hier größtmögliche Magnetfeld $B=12 \mathrm{~T}$ auf 1 normiert. Dabei gehen in die rote Kurve die tatsächlichen Dingletemperaturen $T_{\mathrm{D}}(\mathrm{Lu})=3.5 \mathrm{~K}$ und $T_{\mathrm{D}}(\mathrm{Y})=2.5 \mathrm{~K}$ [Hei95] ein, während zur Berechnung der blauen Kurve für beide Systeme die mittlere Dingletemperatur von $3 \mathrm{~K}$ verwendet wurde. Aus beiden Auftragungen folgt, daß durch die nahezu vorliegende Verdopplung von $m_{1}$ eine mit sinkendem Feld immer stärkere relative Dämpfung im Lu-System gegenüber $\mathrm{YNi}_{2} \mathrm{~B}_{2} \mathrm{C}$ zu erwarten ist. Diese relative Dämpfung wird durch Berücksichtigung der tatsächlichen Dingletemperaturen in der roten Kurve noch verstärkt. So ist die theoretisch mögliche Meßamplitude bei $B \approx 6.5 \mathrm{~T}$, dem Einsetzen des "Peakeffektes“ in $\mathrm{LuNi}_{2} \mathrm{~B}_{2} \mathrm{C}$, bereits auf etwa $40 \%$ gegenüber dem Y-System abgesunken. Betrachtet man das mit dem „Peakeffekt“ verbundene zusätzliche induktive Signal als Störung durch ein 


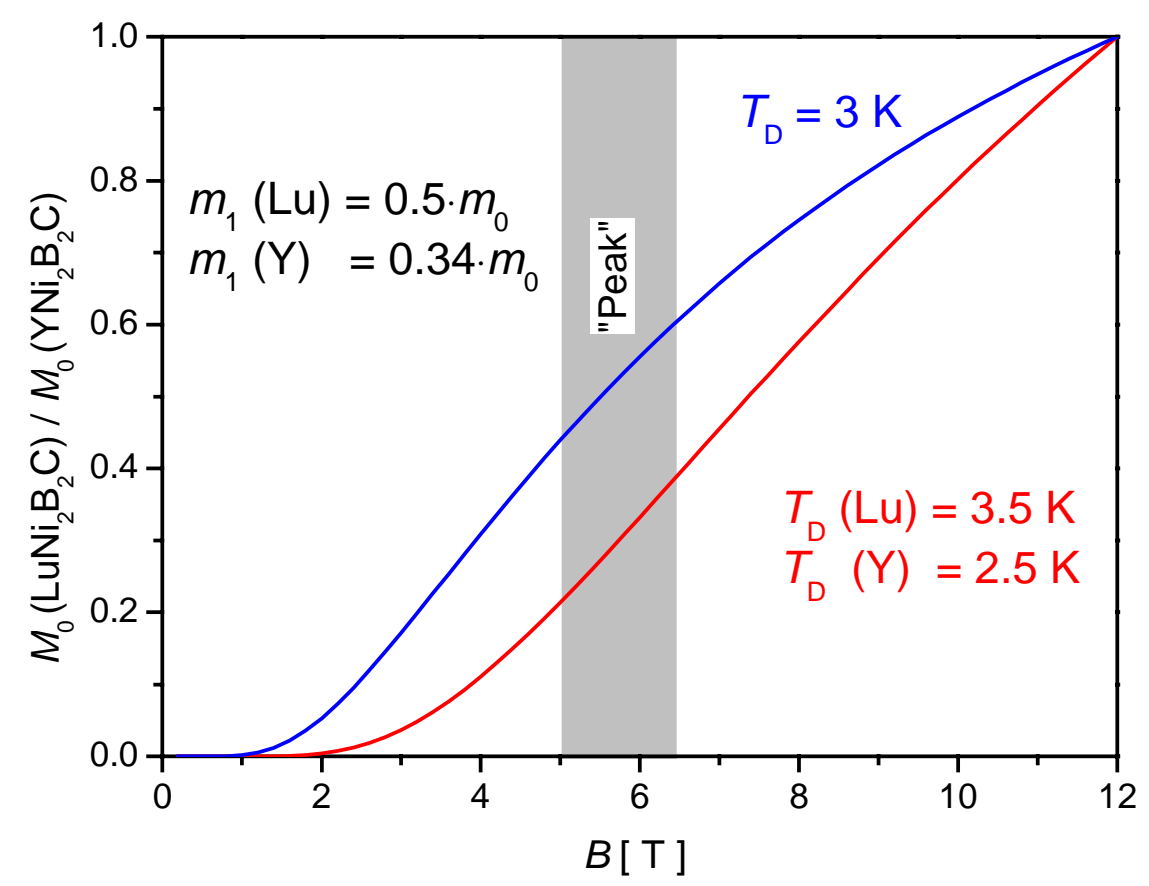

Abb.3.22 Berechnetes Verhältnis der in $\mathrm{LuNi}_{2} \mathrm{~B}_{2} \mathrm{C}$ und $\mathrm{YNi}_{2} \mathrm{~B}_{2} \mathrm{C}$ zu beobachtenden Amplituden für die Frequenzen $F_{1}$ aus der LK-Formel und Gl.3.34. Blau dargestellt der Verlauf für gleiche Probenqualitäten $T_{\mathrm{D}}=3 \mathrm{~K}$. Rot der Verlauf für $T_{\mathrm{D}}\left(\operatorname{LuNi}_{2} B_{2} C\right)=3.5 \mathrm{~K}$ und $T_{\mathrm{D}}\left(Y_{\mathrm{Ni}} B_{2} C\right)=2.5 \mathrm{~K}$

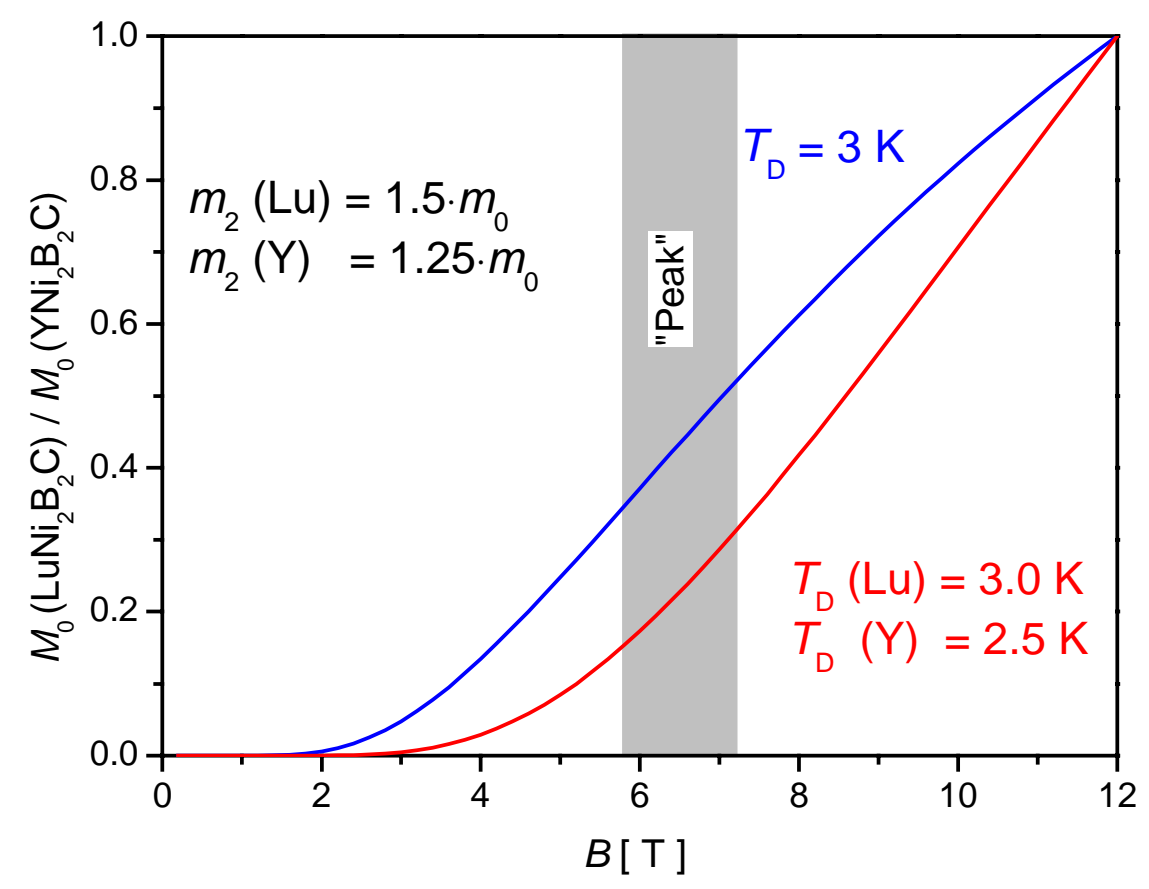

Abb.3.23 Berechnetes Verhältnis der in $\mathrm{LuNi}_{2} \mathrm{~B}_{2} \mathrm{C}$ und $\mathrm{YNi}_{2} \mathrm{~B}_{2} \mathrm{C}$ zu beobachtenden Amplituden für die Frequenzen $F_{2}$ aus der LK-Formel unter der Annahme $J_{2}=$ konst $=1$. Blau dargestellt der Verlauf für gleiche Probenqualitäten $T_{\mathrm{D}}=3 \mathrm{~K}$. Rot der Verlauf für $T_{\mathrm{D}}\left(\mathrm{LuNi}_{2} \mathrm{~B}_{2} \mathrm{C}\right)=3.0 \mathrm{~K}$ und $T_{\mathrm{D}}\left(Y \mathrm{Ni}_{2} \mathrm{~B}_{2} \mathrm{C}\right)=2.5 \mathrm{~K}$ 
möglicherweise überhöhtes Pinning (siehe Kap.3.3.4.3), das unterhalb $5 \mathrm{~T}$ wieder verschwindet, so könnten für tiefere Felder wieder Oszillationen mit einer Amplitude von allerdings nur etwa 20\% verglichen mit der im Y-System erwartet werden. Selbst in $\mathrm{YNi}_{2} \mathrm{~B}_{2} \mathrm{C}$ sind unter ähnlichen Meßbedingungen für Felder $B \approx 6 \mathrm{~T}$ aber nur Amplituden $U_{\mathrm{dHvA}} \approx 5 \mu \mathrm{V}$ möglich, so daß für die zu erwartenden Oszillationen in $\mathrm{LuNi}_{2} \mathrm{~B}_{2} \mathrm{C}$ unterhalb $5 \mathrm{~T}$ die Meßauflösung des Detektorsystems unterschritten ist. Abhilfe aus dieser durch den Anstieg der effektive Masse $m_{1}$ verglichen zum Y-System erzeugten Amplitudenreduktion könnten nur bessere und größere Proben schaffen (siehe Gl.3.11 Gl.3.13). Gemäß $U_{\mathrm{dHvA}} \propto V_{\text {Probe }}$ und $\tau \propto 1 / T_{\mathrm{D}}$ bzw. $R R R \propto \tau$ kann man abschätzen, daß Proben mit etwa fünffachem Volumen oder etwa verdoppeltem Restwiderstandsverhältnis $(R R R \approx 60)$ vorliegen müssen, wobei mit den bisherigen EK-Zucht-Versuchen beides nicht zu realisieren war (siehe Kap.2.1.2 und 2.2.1)

Für die Frequenz $F_{2}=7.755 \mathrm{kT}$ konnten keine dHvA-Oszillationen im supraleitenden Zustand beobachtet werden. Dieser Gegensatz zur verwandten Frequenz $F_{2}=6.93 \mathrm{kT}$ in $\mathrm{YNi}_{2} \mathrm{~B}_{2} \mathrm{C}$ scheint ebenfalls in der erhöhten effektiven Masse $m_{2}$ verglichen $\mathrm{zu} \mathrm{YNi}_{2} \mathrm{~B}_{2} \mathrm{C}$ begründet zu sein. Abb.3.23 zeigt ähnlich dem für $F_{1}$ erklärten Vorgehen die Feldabhängigkeit des Verhältnisses der berechneten Amplituden für das Lu- und das Y-System mit $m_{2}(\mathrm{Lu})=1.5 \cdot m_{0}$ und $m_{2}(\mathrm{Y})=1.25 \cdot m_{0} \quad[\mathrm{Hei} 95]$ bei sonst gleichen Meßbedingungen $T=0.4 \mathrm{~K}$. Neben der Normierung der bei $B$-Modulation eingehenden Dämpfungsfaktoren $R_{\mathrm{T}}=R_{\mathrm{D}}=1$ für $B=12 \mathrm{~T}$ ist der feldabhängige Vorfaktor $J_{2}\left(y \propto 1 / B^{2}\right)$ der Besselfunktion konstant auf 1 gesetzt (vergleiche Gl.3.26). Die vor allem vom größeren $m_{2}$ in $\mathrm{LuNi}_{2} \mathrm{~B}_{2} \mathrm{C}$ bestimmte Feldabhängigkeit der relativen Dämpfung ist der für den T-Modulationsfall ähnlich und wird auch hier durch die größere Dingletemperatur in $\operatorname{LuNi}_{2} \mathrm{~B}_{2} \mathrm{C}\left(T_{\mathrm{D}}=3.0 \mathrm{~K}\right)$ gegenüber dem Y-System $\left(T_{\mathrm{D}}=2.5 \mathrm{~K}\right.$ [Hei95]) noch verstärkt. $\mathrm{Zu}$ berücksichtigen ist weiterhin die bei $B$-Modulation stark reduzierte Amplitude durch die niedrigeren Modulationsfrequenzen (vergleiche Abb.3.4 und Abb.3.10. bzw. Gl.3.25 und G1.3.27). So liegen die vom $\mathrm{YNi}_{2} \mathrm{~B}_{2} \mathrm{C}$ bekannten Amplituden $U_{\mathrm{dHvA}}$ im Feldbereich um $B_{\mathrm{c} 2}(\mathrm{Lu})=7.7 \mathrm{~T}$ bei nur etwa $1 \mu \mathrm{V}$. Betrachtet man das relative Dämpfungsverhältnis von $30 \%$ bei $7.7 \mathrm{~T}$ so wird klar, wie schwierig evtl. auftretende Oszillationen der Frequenz $F_{2}$ in $\mathrm{LuNi}_{2} \mathrm{~B}_{2} \mathrm{C}$ nachzuweisen sind. Aus dieser Argumentation kann vermutet werden, daß es sich auch bei der von $A_{2}$ in $\mathrm{LuNi}_{2} \mathrm{~B}_{2} \mathrm{C}$ gebildeteten Fermifläche um eine solche mit 
teilweise verschwindender Energielücke handeln könnte, wenn die Äquivalenz von $A_{2}(\mathrm{Lu})$ und $A_{2}(\mathrm{Y})$ zutrifft. Der Beweis dieser Vermutung kann aus obigen Gründen nicht mit den hier durchgeführten dHvA-Experimenten erbracht werden.

Aus der Diskussion der Dämpfungsfaktoren mit den eingehenden effektiven Massen folgt allgemein, daß im $\mathrm{LuNi}_{2} \mathrm{~B}_{2} \mathrm{C}$ deutlich schlechtere Bedingungen für die Beobachtung des dHvA-Effekts im supraleitenden Zustand vorliegen. Erschwerend kommt noch das deutlich niedrigere $B_{\mathrm{c} 2}=7.7 \mathrm{~T}$ verglichen mit $B_{\mathrm{c} 2}(\mathrm{Y})=10.5 \mathrm{~T}$ und eine ebenfalls bei niedrigeren Feldern einsetzende ,peakförmige“ Signalvariation unterhalb $B_{\mathrm{c} 2}$ hinzu.

Für die Transporteigenschaften der Elektronen sind natürlich die Elektronen mit großem Wellenvektor $k_{\mathrm{F}}$ in großen Fermiflächenquerschnitten nahe der Brioullinzonengrenze am interessantesten. Die hier speziell für niedrigere Felder nahe $B_{\mathrm{c} 2}$ des Lu-Systems geführte Diskussion der Amplitudenreduktion für zunehmende effektive Massen gilt allerdings auch in abgeschwächter Form für hohe Magnetfelder. Falls die größeren in $\mathrm{YNi}_{2} \mathrm{~B}_{2} \mathrm{C}$ für $B>12 \mathrm{~T}$ beobachteten Fermiflächenquerschnitte [Ngu96] mit effektiven Massen $m_{3}\left(F_{3}=11.7 \mathrm{kT}\right)=3.3 \cdot m_{0}, \quad m_{4}\left(F_{4}=5.8 \mathrm{kT}\right)=3.7 \cdot m_{0}$ auch in äquivalenter Form in $\mathrm{LuNi}_{2} \mathrm{~B}_{2} \mathrm{C}$ vorliegen, so sind diese bei ähnlicher Massezunahme wie bei $A_{1}$ und $A_{2}$ noch schwerer zu beobachten.

Mit der Ähnlichkeit der experimentell bestimmten Fermiflächenquerschnitte $A_{1}$ und $A_{2}$ in den Systemen $\mathrm{YNi}_{2} \mathrm{~B}_{2} \mathrm{C}$ und $\mathrm{LuNi}_{2} \mathrm{~B}_{2} \mathrm{C}$ Systeme überträgt sich auch die für $\mathrm{YNi}_{2} \mathrm{~B}_{2} \mathrm{C}$ beobachtete Diskrepanz zwischen den aus Bandstrukturrechnungen und den aus dHvAExperimenten folgenden Fermiflächenquerschnitten. Im Y-System werden für Feldrichtungen nahe $B \|[001]$ bis zu 4 Fermiflächenquerschnitte $A_{1}=4.77 \mathrm{~nm}^{-2}, A_{2}=66.53 \mathrm{~nm}^{-2}$, $A_{3}=106.89 \mathrm{~nm}^{-2}$ und $A_{4}=55.56 \mathrm{~nm}^{-2}$ mit aufsteigenden effektiven Massen $m_{1}=0.4(1)$, $m_{2}=1.3(1), m_{3}=3.3(2)$ und $m_{4}=3.7(1)$ in Einheiten von $m_{0}$ beobachtet [Ngu96]. In den Bandstrukturrechnungen schneiden 4 Bänder die Fermienergie (siehe Abb.3.25). Die Schnittflächen durch die aus diesen 4 Bändern gebildeten Fermiflächen lassen sich nur für einen Fall einem aus dHvA-Experimenten bestimmten Extremalquerschnitt zuordnen. 


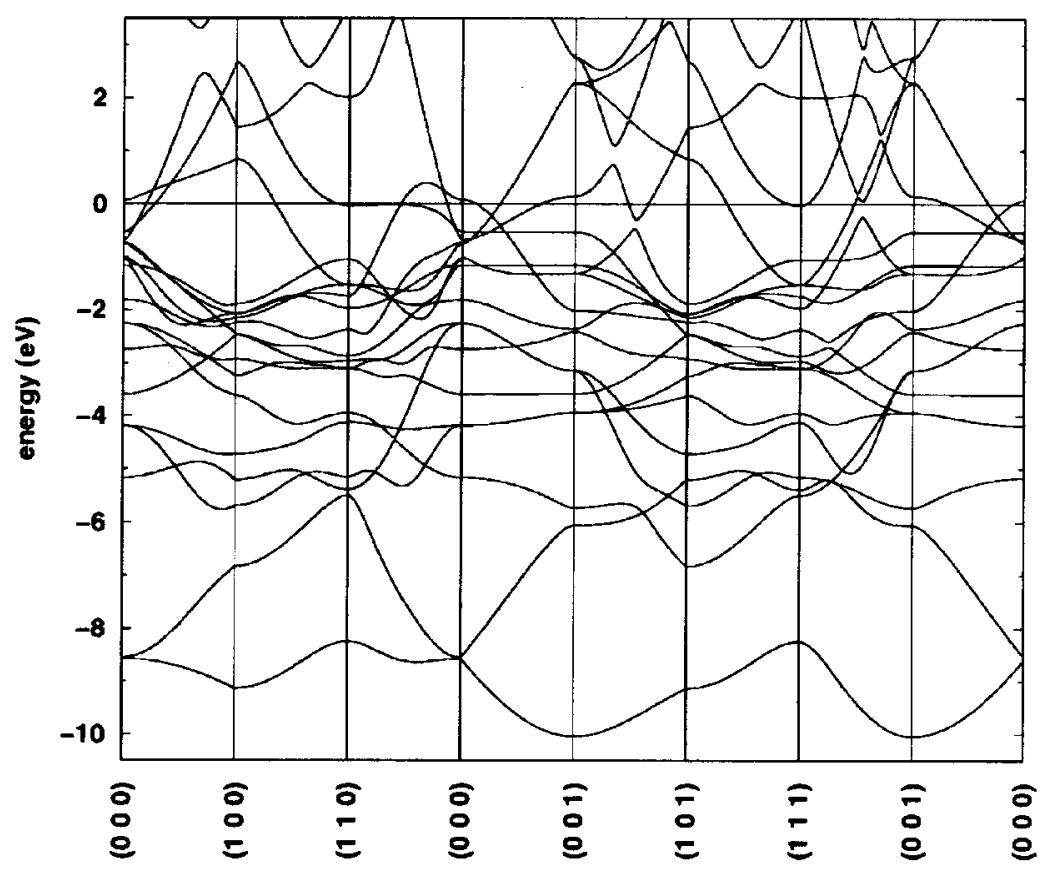

Abb.3.24 Bandstrukturrechnung nach Rosner für das dem $\mathrm{LuNi}_{2} \mathrm{~B}_{2} \mathrm{C}$ verwandte $\mathrm{YNi}_{2} \mathrm{~B}_{2} \mathrm{C}$ mit vier die Fermienergie schneidenden Bändern [Ros00].

So entspricht das experimentelle $A_{3}$ dem maximalen Querschnitt einer entlang der [001]Richtung zylinderartig fortgesetzten Fermifläche um den Punkt $X$ der in Abb.3.25 darge-

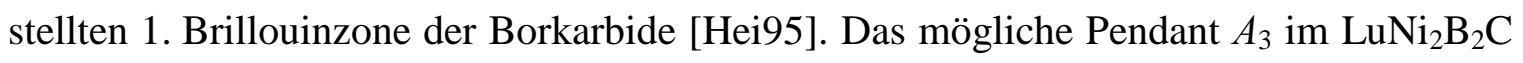
konnte nicht beobachtet werden, was vermutlich an der zu erwartenden größeren effektiven Masse liegt. Der Fermiflächenquerschnitt $A_{1}$ zeigt sowohl im Y-System [Ngu96] als auch im $\mathrm{LuNi}_{2} \mathrm{~B}_{2} \mathrm{C}$ die Winkelabhängigkeit einer zugehörigen ellipsoiden Fermifläche mit langer Achse entlang [001]. Die geringe Größe und die elliptische Form der experimentellen Querschnittsflächen $A_{1}$ stimmen am ehesten mit den Schnittflächen einer kleinen „Elektronentasche“ um den Punkt $\Gamma$ aus verschiedenen Bandstrukturrechnungen überein [Kim95], [Sin95a]. 


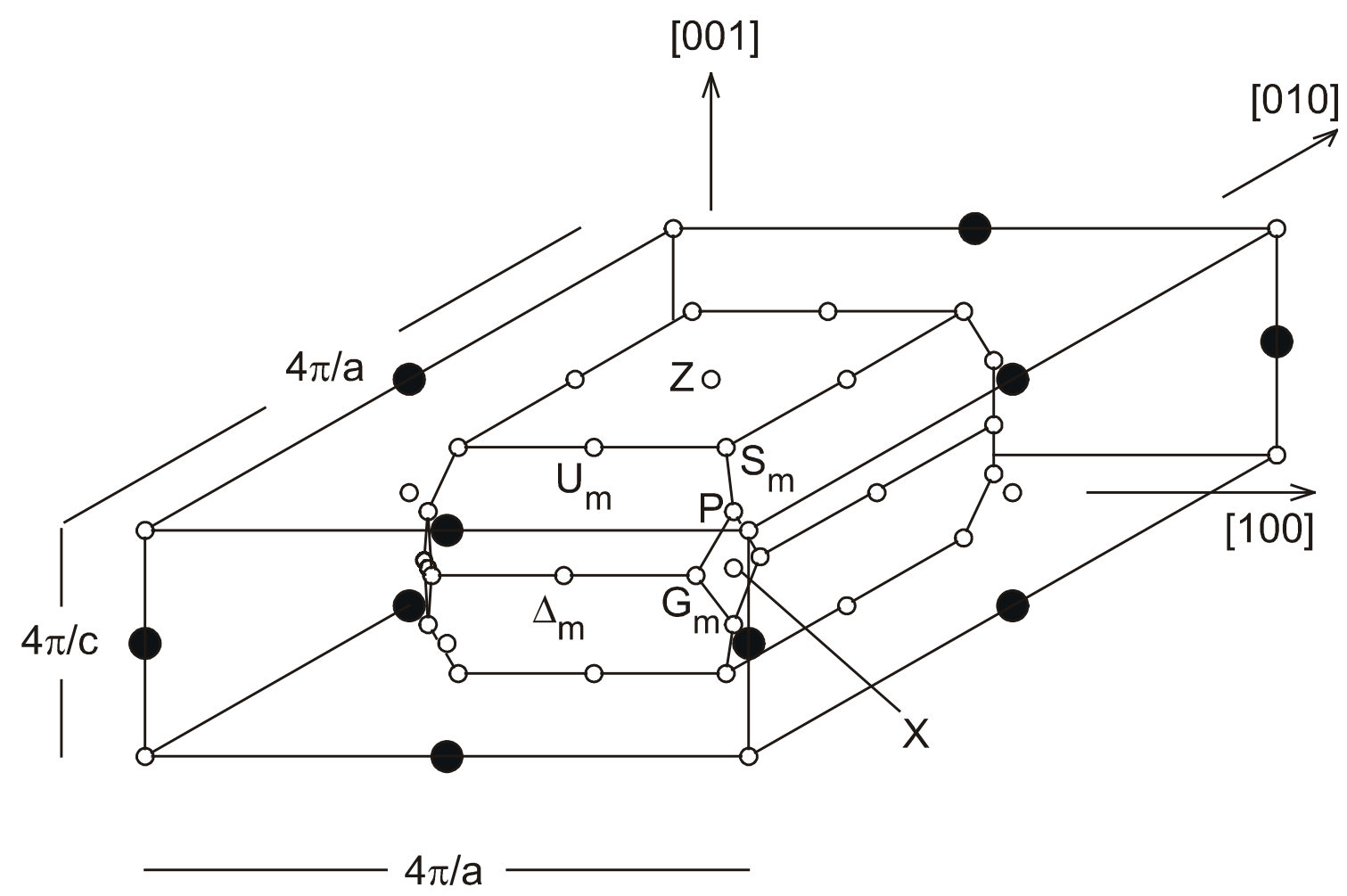

Abb.3.25 Erste Brillouinzone von $\mathrm{LuNi}_{2} \mathrm{~B}_{2} \mathrm{C}$ und $\mathrm{YNi}_{2} \mathrm{~B}_{2} \mathrm{C}$. Die Koordinaten der eingezeichneten Symmetriepunkte in Einheiten der Gitterkonstanten $a=0.347 \mathrm{~nm}$ und $c=1.066 \mathrm{~nm}$ lauten:

$$
\begin{aligned}
& \Gamma=\left(\begin{array}{l}
0 \\
0 \\
0
\end{array}\right), \quad Z=\frac{2 \pi}{c}\left(\begin{array}{l}
0 \\
0 \\
1
\end{array}\right), \quad X=\frac{2 \pi}{c}\left(\begin{array}{c}
c / 2 a \\
c / 2 a \\
0
\end{array}\right), \quad P=\frac{2 \pi}{c}\left(\begin{array}{c}
c / 2 a \\
c / 2 a \\
1 / 2
\end{array}\right), \\
& \Delta_{m}=\frac{2 \pi}{c}\left(\begin{array}{c}
(c / a+a / c) / 2 \\
0 \\
0
\end{array}\right), \quad U_{m}=\frac{2 \pi}{c}\left(\begin{array}{c}
(c / a-a / c) / 2 \\
0 \\
1
\end{array}\right), \quad V=\frac{2 \pi}{c}\left(\begin{array}{c}
c / 2 a \\
0 \\
1 / 2
\end{array}\right), \\
& S_{m}=\frac{2 \pi}{c}\left(\begin{array}{c}
(c / a-a / c) / 2 \\
(c / a-a / c) / 2 \\
1
\end{array}\right), \quad G_{m}=\frac{2 \pi}{c}\left(\begin{array}{c}
(c / a+a / c) / 2 \\
(c / a-a / c) / 2 \\
0
\end{array}\right)
\end{aligned}
$$


Abb.3.26 zeigt eine Folge von [001]-Schnitten nach Rosner [Ros00] durch diese Elektronentasche mit Abständen $n \cdot(1 / 10) \cdot(\pi / c)$ von $\Gamma$. Zum quantitativen Vergleich der Schnittebenenabhängigkeit der rot hervorgehobenen Fermiflächenquerschnitte sind diese in jeder Ebene relativ zu einer quadratischen Fläche $(4 \pi / a)^{2}$ dargestellt. Diese quadratischen Flächen sind in jeder Ebene mehr als doppelt so groß wie die zugehörigen Brillouinzonenschnitte, der für $n=0$ blau mit eingezeichnet ist. Wie zu sehen sind die Schnitte durch die Elektronentasche nahezu rotationssymmetrisch, im Einklang mit der experimentellen Konstruktion einer rotationsellipsoiden Fermifläche aus $A_{1}$. Die nach Rosner berechnete Querschnittsfläche ist für $n=0$ maximal und nimmt mit
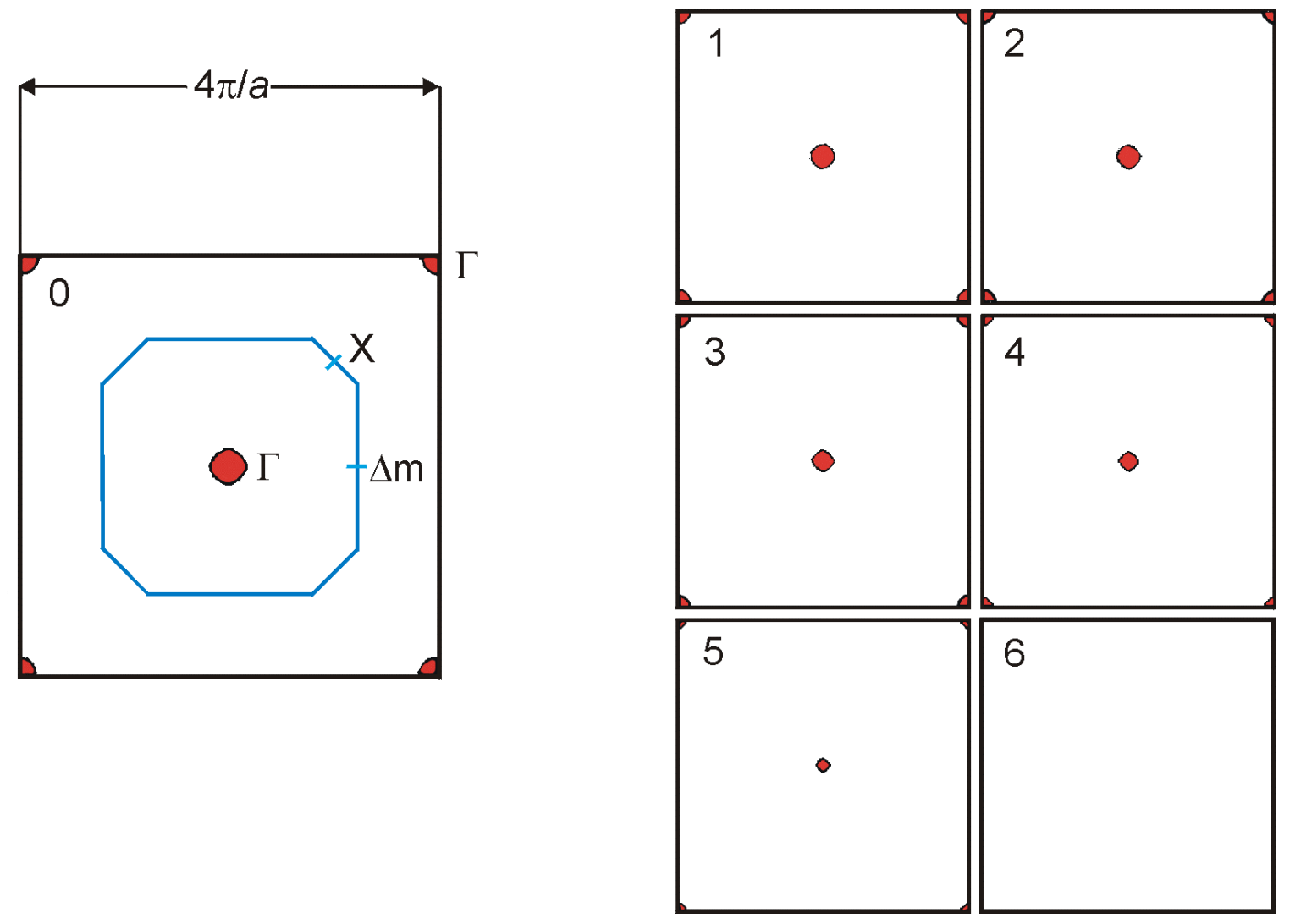

Abb.3.26 Schnitte durch die Fermifläche eines der die Fermienergie schneidenden Bänder [Ros00].

steigendem $n$ betragsmäßig ab, bis sie in der Schnittebene durch 6·(1/10) $(\pi / c)$ ganz verschwunden ist. Für ihre Schnittfläche durch den Ursprung erhält man $A_{1 \mathrm{R}}(a, b) \approx \pi k_{\mathrm{a}}^{2} \approx 6.9 \mathrm{~nm}^{-2}$, was etwa $2.1 \%$ des quadratisch genäherten Brillouinzonenquerschnitts $(2 \pi / a)^{2}$ in dieser Ebene entspricht. Für die Halbachse der Elektronentasche 
entlang der $c$-Richtung gilt genähert $k_{\mathrm{c}} \approx(5.5 / 10) \cdot(\pi / c)$. Somit berechnet man für die als Rotationsellipsoid angenommene Elektronentasche in einer [100]-Schnittebene durch den Ursprung $A_{2 \mathrm{R}}(a, c) \approx \pi k_{\mathrm{a}} \cdot k_{\mathrm{c}} \approx 7.55 \mathrm{~nm}^{-2}$ entsprechend etwa $7.3 \%$ des wiederum quadratisch genäherten Brioullinzonenquerschnitts $(2 \pi / a) \cdot(2 \pi / c)=104.04 \mathrm{~nm}^{-2}$. Damit zeigt die Elektronentasche nach Rosner in etwa das gleiche Verhalten wie die experimentell bestimmte Querschnittsfläche $A_{1}$ mit $A_{1}(a, b)=2.65 \mathrm{~nm}^{-2}$ und $A_{1}(a, c)=4.20 \mathrm{~nm}^{-2}$, allerdings mit einem Halbachsenverhältnis $\left(k_{\mathrm{a}} / k_{\mathrm{c}}\right)($ Rosner $) \approx 0.92$ gegenüber $\left(k_{\mathrm{a}} / k_{\mathrm{c}}\right)($ Exp. $) \approx 0.63$. Die diskutierten Querschnitte liegen allerdings im Rahmen kleiner Querschnitte, deren Ergebnis aus Bandstrukturrechnungen extrem empfindlich von der Wahl der dort verwendeten Parameter (bspw. $E_{\mathrm{F}}$ ) abhängt [Sin95a]. Aufgrund der qualitativen Übereinstimmung in der Geometrie scheint eine Identifikation der experimentellen Querschnitte mit denen der Elektronentasche am wahrscheinlichsten. Für die Querschnittsfläche $A_{2}$ lassen sich keine geschlossenen Flächen der aus Bandstrukturrechnungen folgenden Fermiflächenquerschnitte zuordnen. Dies ist eine weitere Gemeinsamkeit zum Fermiflächenquerschnitt $A_{2}$ im Y-System, dessen Zuordnung zu Fermiflächenquerschnitten aus Bandstrukturrechnungen ebenfalls nicht möglich ist [Hei95]. Die experimentell bestimmten Querschnittsflächen für $A_{2}$ in der Orientierung $B \|$ [001] deuten sowohl in $\mathrm{LuNi}_{2} \mathrm{~B}_{2} \mathrm{C}$ als auch in $\mathrm{YNi}_{2} \mathrm{~B}_{2} \mathrm{C}$ auf eine Identifikation dieser Flächen mit einer aus verschiedenen Bandstrukturrechnungen folgenden Lochbahn um den Punkt $Z$ [Ros00], [Sin95a]. Diese ist aber in den Bandstrukturrechnungen für beide Systeme nicht vollständig geschlossen, kann also nicht für das Signal des experimentell bestimmten Querschnitts verantwortlich sein.

Positiver zu bewerten ist der Vergleich der aus den dHvA-Experimenten bestimmten Fermigeschwindigkeiten mit einer mittleren Fermigeschwindigkeit aus Bandstrukturrechnungen. So stimmt die experimentell ermittelte Fermigeschwindigkeit $v_{\mathrm{F} 1}=1.93 \cdot 10^{5} \mathrm{~m} / \mathrm{sec}$ sehr gut mit einer aus Bandstrukturrechnungen folgenden Angabe [Pic94] für eine mittlere Fermigeschwindigkeit in [100]-Richtung $v_{\mathrm{F}, \mathrm{a}}=2.12 \cdot 10^{5} \mathrm{~m} / \mathrm{sec}$ überein. Weiterhin bilden die Fermigeschwindigkeiten aus dHvA-Experimenten eine wichtige experimentelle Rechtfertigung für ein im isotropen Zwei-Band-Modell entworfenes Supraleitungsmodell für die Borkarbide nach Shulga und Drechsler [Shu98], auf das im folgenden noch eingegangen wird. Nach Bandstrukturrechnungen von Drechsler 
[Dre99] sind die in $\mathrm{LuNi}_{2} \mathrm{~B}_{2} \mathrm{C}$ und $\mathrm{YNi}_{2} \mathrm{~B}_{2} \mathrm{C}$ auftretenden Fermigeschwindigkeiten grob in 2 Klassen einzuordnen, deren relative Größe sich etwa um den Faktor 6 unterscheidet. Ebenso wie die Elektronen mit $v_{\mathrm{F} 1}$, seien demnach die mit $v_{\mathrm{F} 2}$ der Klasse der „schnelleren“"Elektronen zuzuordnen.

Neben den diskutierten Ähnlichkeiten und Unterschieden mit Ergebnissen am Y-System bleibt aber allgemein festzuhalten, daß die angestrebte erweiterte Überprüfung der berechneten Fermiflächen aus nur zwei gemessenen Querschnitten an $\mathrm{LuNi}_{2} \mathrm{~B}_{2} \mathrm{C}$ nicht möglich ist.

Die durchgeführten Widerstandsmessungen für Magnetfelder innerhalb der $(a, b)$-Ebene eines hochwertigen $\mathrm{LuNi}_{2} \mathrm{~B}_{2} \mathrm{C}$-EK widersprechen der $B_{\mathrm{c} 2}(T, \alpha)$-Vorhersage aus der Beschreibung im $d$-Wellen-Modell nach Wang [Wan98]. Vielmehr läßt sich die Temperaturabhängigkeit des $B_{\mathrm{c} 2}(T)$-Verlaufes für feste Orientierung $B \|$ [001], mit der ausgeprägten positiven Krümmung für $T \geq 12 \mathrm{~K}$, mit zwei Modellen in konventioneller EliashbergSupraleitungstheorie beschreiben. Der in Abb.3.21b dargestellte $B_{\mathrm{c}^{2}}$-Verlauf wurde ebenso wie der am angesprochenen $\mathrm{YNi}_{2} \mathrm{~B}_{2} \mathrm{C}$-EK ermittelte anhand eines Zwei-Band-Modells im isotropen Eliashberg-Formalismus beschrieben [Shu98]. Die Grundannahmen für das Zwei-Band-Modell liegen in einer Klassifizierung der in den Borkarbiden vorliegenden Fermigeschwindigkeiten in zwei oder drei Gruppen. Dies ist sowohl durch Bandstrukturrechnungen [Dre99] als auch durch dHvA-Experimente im Hochfeld [Ngu96] begründbar. So findet man in den angesprochenen dHvA-Experimenten für das Y-System grob zwei Klassen von Fermigeschwindigkeiten $v_{\mathrm{Fa}} \approx 4 \cdot 10^{5} \mathrm{~m} / \mathrm{sec}$ und $v_{\mathrm{Fb}} \approx 0.85 \cdot 10^{5} \mathrm{~m} / \mathrm{sec}$. Dabei gehören die Fermiflächenquerschnitte mit $v_{\mathrm{Fb}} \mathrm{zu}$ Elektronen mit vergleichbar großer effektiver Masse $m \approx m_{4}=3.7 \cdot m_{0}$ [Ngu96], weshalb diese vermutlich im verwandten $\mathrm{LuNi}_{2} \mathrm{~B}_{2} \mathrm{C}$ bislang nicht in $\mathrm{dHvA}$-Experimenten beobachtet werden konnten. Aufgrund der bislang im Experiment und den Bandstrukturrechnungen beobachteten Übereinstimmungen in den elektronischen Eigenschaften von $\mathrm{Lu}-$ und $\mathrm{YNi}_{2} \mathrm{~B}_{2} \mathrm{C}$ [Hil99], [Dre99] ist diese Klassifizierung aber vermutlich auf das $\mathrm{System} \mathrm{LuNi}_{2} \mathrm{~B}_{2} \mathrm{C}$ übertragbar. Die im isotropen Zwei-Band-Modell eingehenden beiden Bänder werden eben mit Elektronen aus diesen zwei Klassen von Fermigeschwindigkeiten besetzt, wobei das $v_{\mathrm{Fb}}$-Band als Haupträger der Supraleitung betrachtet werden kann. Die anderen im EliashbergFormalismus eingehenden Größen wie Elektron-Phonon-Kopplungsstärken sind ebenfalls 
experimentell bestimmt [Gom97]. Unter Anwendung des isotropen Zwei-Band-Modells mit stark Elektron-Phonon-koppelndem $v_{\mathrm{Fb}}$-Band, schwacher Kopplung zwischen den Bändern und Einbeziehung der aus experimentellen Dingletemperaturen ([Hei95], diese Arbeit) bestimmten Streuenergien $k_{\mathrm{B}} T_{\mathrm{D}}$ erhält man die in Abb.3.27 rot dargestellte Anpassung an die experimentellen $B_{\mathrm{c} 2}(T)$-Daten. Die Übereinstimmung zwischen experimentellem Verlauf und theoretischer Beschreibung ist als sehr gut anzusehen, gleiches gilt für die entsprechenden Daten zum $\mathrm{YNi}_{2} \mathrm{~B}_{2} \mathrm{C}$ [Shu98].

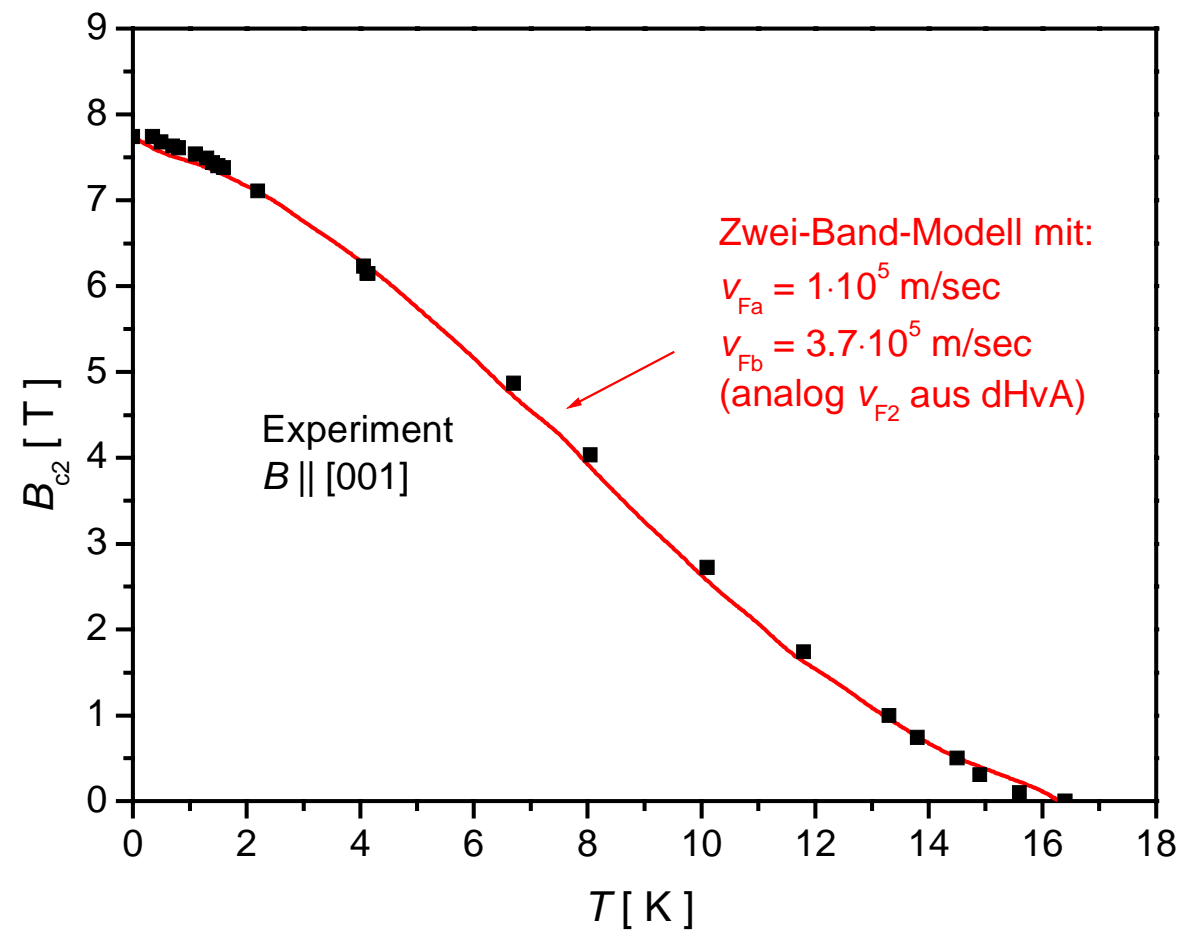

Abb.3.27 Anpassung des $B_{\mathrm{c} 2}$-Verlaufes im isotropen Zwei-Band-Modell nach Shulga et al. [Shu98].

Eine ähnlich gute Übereinstimmung mit $B_{\mathrm{c} 2}(T)$ aus Wärmekapazitätsmessungen an Polykristallen wurde kürzlich in der im Eliashberg-Formalismus äquivalenten Beschreibung [Shu98] im anisotropen Ein-Band-Modell [Man00] erreicht. Damit sprechen die $B_{\mathrm{c} 2^{-}}$ Daten in den unmagnetischen Borkarbiden eindeutig für einen konventionellen Supraleitungsmechanismus.

Prinzipiell ist eine Ausweitung der Beschreibung der $B_{\mathrm{c}^{2}}$-Verläufe im Rahmen des Eliashberg-Formulismus mit detaillierterem Eingehen der anisotropen Fermifläche denkbar [Ros00]. Damit wäre evtl. auch die beobachtete Anisotropie in der $(a, b)$-Ebene be- 
schreibbar. Dazu ist eine Ausweitung der experimentellen Kenntnisse über die Fermifläche der Borkarbide aber unumgänglich [Ros00]. 


\section{$4 \mathrm{DyNi}_{2} \mathrm{~B}_{2} \mathrm{C}$}

\subsection{Vorarbeiten aus der Literatur}

Die zweite untersuchte $S E \mathrm{Ni}_{2} \mathrm{~B}_{2} \mathrm{C}$-Verbindungen ist das System $\mathrm{DyNi}_{2} \mathrm{~B}_{2} \mathrm{C}$, das bei $T_{\mathrm{N}}=10.3 \mathrm{~K}$ antiferromagnetisch $(\mathrm{afm})$ ordnet und im geordneten Zustand bei $T_{\mathrm{c}}=6.5 \mathrm{~K}$ supraleitend wird [Lyn97], [Pen99]. Es zählt neben $\mathrm{HoNi}_{2} \mathrm{~B}_{2} \mathrm{C}$ und $\mathrm{ErNi}_{2} \mathrm{~B}_{2} \mathrm{C}$ zu den Borkarbiden mit einem Verhältnis der Ordnungstemperaturen $r=T_{\mathrm{N}} / T_{\mathrm{c}}$ nahe bei 1 , d.h. es existieren stark konkurrierende magnetische und supraleitende Effekte. So wird sowohl in $\mathrm{HoNi}_{2} \mathrm{~B}_{2} \mathrm{C}$ als auch in $\mathrm{DyNi}_{2} \mathrm{~B}_{2} \mathrm{C}$ ein durch die magnetische Ordnung bewirktes ,reentrant"-Verhalten im elektrischen Widerstand beobachtet [Kru96], [Pen99]. Die nötige Prozeßführung zur Beobachtung von einsetzender Supraleitung in $\mathrm{DyNi}_{2} \mathrm{~B}_{2} \mathrm{C}$ während eines Heizexperiments ist aus dem von Peng mittels resistiven Messungen bestimmten B/T-Phasendiagramm abzulesen (siehe Abb.4.1). Das Phasendiagramm ergibt für $B$ || [100] eine ausgeprägte Hysterese der dort mit $M 1$ und $M 3$ bezeichneten feldinduzierten magnetischen Phasen in Verbindung mit starker Hysterese in $B_{\mathrm{c} 2}(T)$ unterhalb $T=2.0 \mathrm{~K}$. Beendet man somit ein Hystereseexperiment bei tiefster Temperatur und von Null verschiedenem Feld $B \approx 200 \mathrm{mT}$, kann man die Probe bei einer anschließenden Temperaturerhöhung bei $B=$ konst vom normalleitenden in den supraleitenden Zustand überführen.
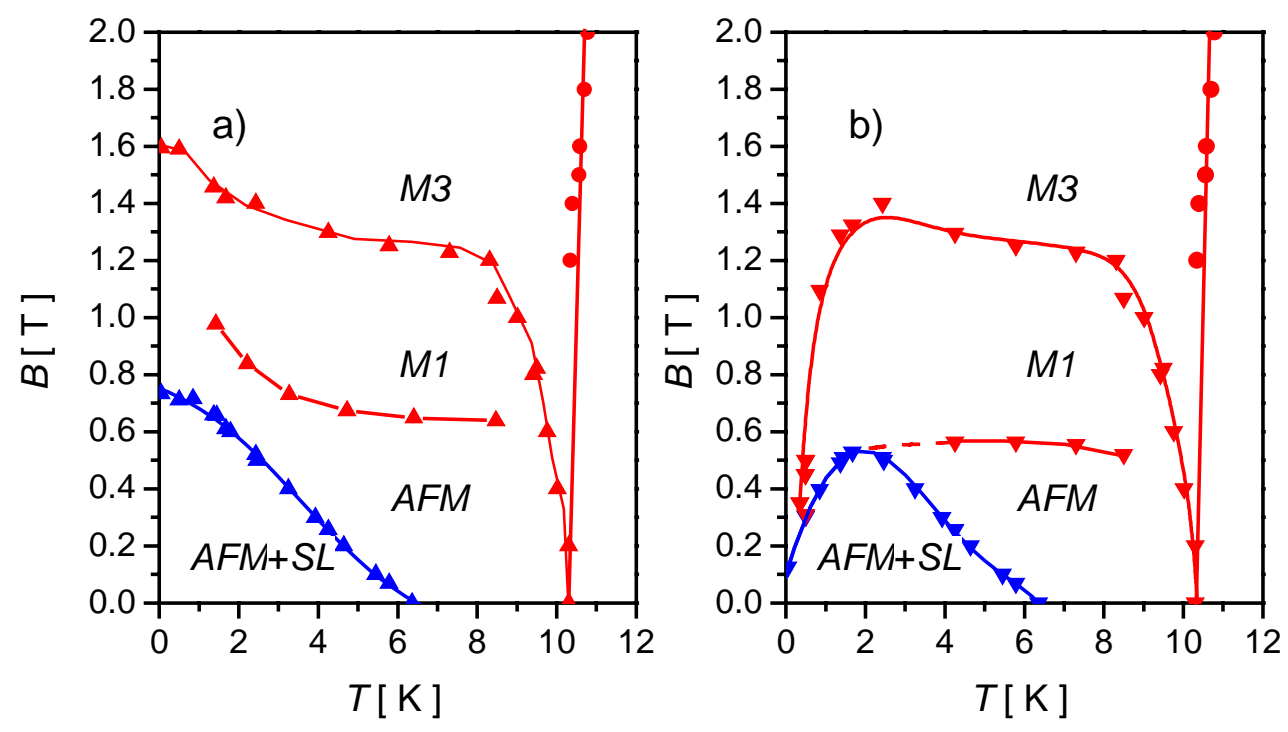

Abb.4.1 B/T-Phasendiagramm von $\mathrm{DyNi} \mathrm{i}_{2} \mathrm{~B}_{2} \mathrm{C}$ für a) Felderhöhung und b) Felderniedrigung in der Orientierung $B \|$ [100] nach [Pen99]. Blau eingezeichnet die Phasengrenze zur Supraleitung und rot magnetische Phasengrenzen. 
Dieser ,reentrant“-Effekt der Supraleitung konnte in der Orientierung $B \|$ [110] mit Abfolge der Phasengrenzen $A F M \leftrightarrow M 2 \leftrightarrow F M$ nicht beobachtet werden. Auch dort gibt es ähnlich $B \|[100]$ zu tiefen Temperaturen einen Anstieg der Hysterese in den magnetischen Phasenübergängen, allerdings nur mit geringen Auswirkungen auf den $B_{\mathrm{c} 2}$-Verlauf [Pen99].

Das Verständnis solcher Anisotropieeffekte erfordert die detaillierte Untersuchung der felderzeugten magnetischen Phasen ausgehend vom afm Grundzustand. Die mikroskopische Charakterisierung des afm Zustandes mit elastischer Neutronenstreuung ergibt die in Abb.1.2 dargestellte Abfolge von $f m$ ausgerichteten Dy-Momenten innerhalb der $(a, b)$ Ebenen und $a f m$ Kopplung zwischen benachbarten $(a, b)$-Ebenen entlang der [001]Richtung [Lyn97]. Während die afm Kopplung über die RKKY-WW übertragen wird [Lyn97] ist die Kristallfeldaufspaltung (Größenordnung $\Delta E / \mu(S E) \approx 60 \mathrm{~T}$ [Ka198]) entscheidend für die Anordnung der $S E$-Momente in <110>-Richtungen. Sie ist auch der Grund, daß für Magnetfelder entlang der [001]-Richtung keine feldinduzierten magnetischen Phasen beobachtet werden, d.h. $\mathrm{DyNi}_{2} \mathrm{~B}_{2} \mathrm{C}$ verbleibt für diese Feldorientierungen im afm Zustand. Dies rechtfertigt die im folgenden implizierte Annahme von verschwindender Magnetisierung entlang der [001]-Richtung der Proben.

Aus Experimenten mit Magnetfeldrichtungen innerhalb der $(a, b)$-Ebene und $T<T_{\mathrm{N}}$ findet man aus verschiedenen Quellen eine unterschiedliche Anzahl von feldinduzierten metamagnetischen Phasen zwischen dem afm Grundzustand und den Sättigungsphasen für $B=2$ T [Nau98], [Can97a], [Pen99]. Die Ergebnisse aus Magnetisierungsmessungen für $B \|$ [110] und $B \|$ [100] [Can97a], [Nau98] lassen auf Sättigungsmagnetisierungen ${ }^{\natural} \mathrm{der}$ für $B=2$ T erzeugten Zustände von

$$
\begin{aligned}
& \mu_{110}(B=2 \mathrm{~T})=9.7 \cdot \mu_{\mathrm{B}}=\mu\left(\mathrm{Dy}^{3+}\right)=\mu_{\mathrm{sat}} \quad \text { und } \\
& \mu_{100}(B=2 \mathrm{~T})=(1 / \sqrt{ } 2) \cdot 9.7 \cdot \mu_{\mathrm{B}}=(1 / \sqrt{ } 2) \cdot \mu_{\mathrm{sat}}
\end{aligned}
$$

schließen. Dabei ist 9.7 $\mu_{\mathrm{B}}$ das magnetische Moment $\mu\left(\mathrm{Dy}^{3+}\right)$ des 3-wertig positiven DyIons aus Squid-Messsungen an einem $\mathrm{DyNi}_{2} \mathrm{~B}_{2} \mathrm{C}-\mathrm{EK}$ [Nau98]. Somit sind für $B \|[110]$

\footnotetext{
${ }^{4} \mu$ meint im folgenden die Magnetisierung pro $\mathrm{Dy}^{3+}$-Ion. Die anderen Konstituenten tragen in den Borkarbiden nicht zur Magnetisierung bei. Somit ergibt sich die Probenmagnetisierung $M$ aus der Anordnung der Dy-Momente.
} 
alle magnetischen Momente entlang der in Feldrichtung weisenden magnetisch leichten Richtung gesättigt, es liegt eine gesättigte ferromagnetische Phase $F M$ entlang der [110]Richtung vor. Die beobachtete Sättigungsmagnetisierung für $B \|[100]$ spricht für eine Erhaltung von festen <110>-Richtungen der magnetischen Momente, die zur Erzeugung von $\mu_{100}=(1 / \sqrt{ } 2) \cdot 9.7 \cdot \mu_{\mathrm{B}}$ auf die leichte [110]- und [1 $\left.\overline{1} 0\right]$-Richtung verteilt sein müssen. Dies ist also die Sättigungsmagnetisierung der in Abb.4.1 mit M3 bezeichneten Phase mit Magnetisierungsrichtung entlang [100].

Eine erste Beschreibung der Struktur der metamagnetischen Phasen M1 und M2 zwischen dem für kleine Magnetfelder beobachteten AFM und den für Felder um 2.0 T beobachteten M3 bzw. FM entwickelten Winzer et al. [Win99b] aus experimentell gewonnenen Winkelabhängigkeiten der Phasengrenzen für Magnetfelder $B \|(a, b)$-Ebene (siehe Abb.4.2).

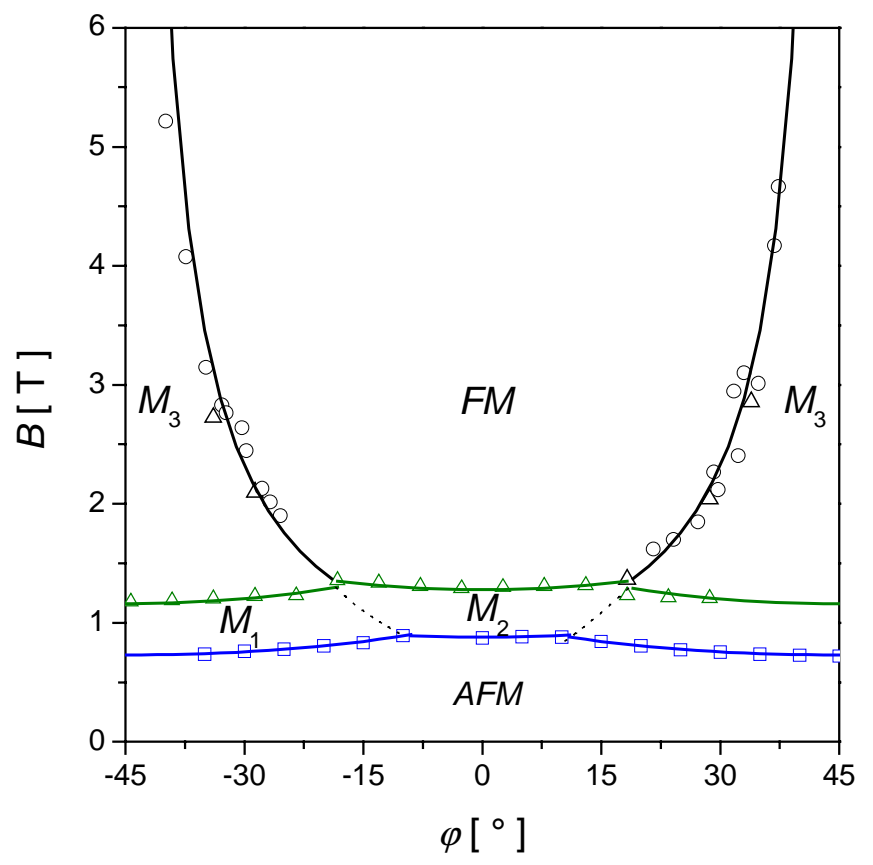

Abb.4.2 Magnetisches Phasendiagramm für T = 1.4 K nach [Win99b]. Symbole stammen aus experimentell gewonnene Phasengrenzen und Linien beschreiben berechnete Phasengrenzen nach dem im Text erklärten Verfahren.

Demnach beobachtet man bei der Temperatur $T=1.4 \mathrm{~K}$ für die Phasengrenzen im Winkelbereich $\varphi \angle(B,[110]) \leq 9^{\circ}$ um $B \|[110]$ eine Abhängigkeit $B_{\text {grenz }}(110) \propto 1 / \cos (\varphi)$ und im Winkelbereich $\gamma=\left(45^{\circ}-\varphi\right) \leq 27^{\circ}$ um die Orientierung $B \|[100]$ eine Abhängigkeit 
$B_{\text {grenz }}(100) \propto 1 / \cos \left(45^{\circ}-\varphi\right)$. Weiterhin findet man für die Phasengrenze zwischen der gesättigten Phase $F M$ und $M 3$ die experimentelle Abhängigkeit $B_{\text {grenz }}(F M, M 3) \propto 1 / \cos \left(\varphi+45^{\circ}\right)$. Unter Beibehaltung leichter <110>-Richtungen der magnetischen Momente in allen feldinduzierten Phasen und $f m$ Kopplung der Momente innerhalb einer $(a, b)$-Ebene lassen sich diese beobachteten Winkelabhängigkeiten aus der Energiebetrachtung $E=\vec{\mu} \cdot \vec{B}$ analytisch gewinnen. In der Magnetfeldrichtung $B \|[100]$ wird die feldinduzierte metamagnetische Zwischenphase $M 1$ eine Magnetisierungsrichtung entlang [100] aufweisen, da keine der leichten Richtungen [110] oder [1 $\overline{1} 0$ ] für die Dy-Momente ausgezeichnet ist. D.h. die Magnetisierungsrichtung der Zwischenphase $M_{1}$ weist entlang [100], aber ihr Betrag wird kleiner als $\mu_{100}=(1 / \sqrt{ } 2) \mu_{\text {sat }}$ sein. Entsprechend darf für die metamagnetische Phase $M 2$ zwischen der $a f m$ und der $f m$ Phase für $B$ || [110] angenommen werden, daß ihre Magnetisierungsrichtung entlang der [110]-Richtung weist, allerdings mit kleinerem Betrag als $\mu_{\text {sat }}$.

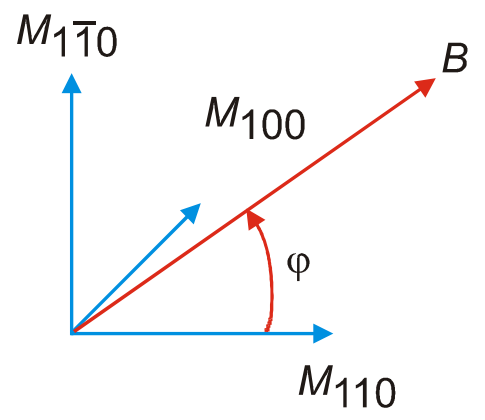

Abb.4.3 Geometrie zu der im Text beschriebenen Berechnung der feld-und orientierungsabhängigen Gibbschen Freien Enthalpie.

Mit dieser Argumentation zur Magnetsierungsrichtung in den felderzeugten Phasen gilt für die Feld- und Winkelabhängigkeit ihrer Gibbschen Freien Enthalpie $G$ (siehe Abb.4.3):

Gl.4.1 $\quad G=$ konst $-\vec{\mu} \cdot \vec{B}=$ konst $-\mu \cdot B \cdot \cos (\angle(\mu, B))$

$\Rightarrow \quad G(A F M)=$ konst $_{\mathrm{AFM}}-\mu_{\mathrm{AFM}} \cdot B=$ konst $_{\mathrm{AFM}}-0$ 


$$
\begin{aligned}
G(M 1) & =\text { konst }_{\mathrm{M} 1}-\mu_{\mathrm{M} 1} \cdot B \cos \left(45^{\circ}-\varphi\right) \\
G(M 3) & =\text { konst }_{\mathrm{M} 3}-\mu_{\mathrm{M} 3} \cdot B \cos \left(45^{\circ}-\varphi\right)=\text { konst }_{\mathrm{M} 3}-(1 / \sqrt{ } 2) \mu_{\mathrm{sat}} \cdot B \cos \left(45^{\circ}-\varphi\right) \\
& =\text { konst }_{\mathrm{M} 3}-(1 / 2) \mu_{\mathrm{sat}} B \cos \left(90^{\circ}-\varphi\right)-(1 / 2) \mu_{\mathrm{sat}} B \cos (\varphi) \\
& =\text { konst }_{\mathrm{M} 3}-(1 / 2) \mu_{\mathrm{sat}} B \sin (\varphi)-(1 / 2) \mu_{\mathrm{sat}} B \cos (\varphi) \\
G(M 2) & =\text { konst }_{\mathrm{M} 2}-\mu_{\mathrm{M} 2} \cdot B \cos (\varphi) \\
G(F M) & =\text { konst }_{\mathrm{FM}}-\mu_{\mathrm{FM}} \cdot B \cos (\varphi)=\text { konst }_{\mathrm{FM}}-\mu_{\mathrm{sat}} \cdot B \cos (\varphi)
\end{aligned}
$$

An der Phasengrenze $B_{\text {Phase1Phase2 }}$ benachbarter magnetischer Phasen müssen ihre Energien gleich sein, so daß gilt (siehe Abb.4.2):

Gl. $4.2 \quad G(A F M) \stackrel{!}{=} G(M 1)$

$\Rightarrow \quad$ konst $_{\mathrm{AFM}}=$ konst $_{\mathrm{M} 1}-M_{\mathrm{M} 1} \cdot B_{\mathrm{AFM} / \mathrm{M} 1} \cos \left(45^{\circ}-\varphi\right)$, d.h.

$$
B_{\mathrm{AFMM} 1}=\text { konst }_{1} /\left(\cos \left(45^{\circ}-\varphi\right)\right)
$$

Ensprechend folgt aus

$$
\begin{aligned}
& G(M 1) \stackrel{!}{=} G(M 3) \\
& \Rightarrow \quad B_{\mathrm{M} 1 \mathrm{M} 3}=\text { konst }_{3} /\left(\cos \left(45^{\circ}-\varphi\right)\right) \\
& G(A F M) \stackrel{!}{=} G(M 2) \\
& \Rightarrow \quad B_{\mathrm{AFMM} 2}=\text { konst }_{2} /(\cos (\varphi)) \\
& G(M 2) \stackrel{!}{=} G(F M) \\
& \Rightarrow \quad B_{\mathrm{M} 2 \mathrm{FM}}=\text { konst }_{4} /(\cos (\varphi))
\end{aligned}
$$




$$
\begin{aligned}
& G(M 3) \stackrel{!}{=} G(F M) \\
\Rightarrow \quad & B_{\mathrm{M} 3 \mathrm{FM}}=\text { konst }_{5} /\left(\cos \left(\varphi-45^{\circ}\right)\right)
\end{aligned}
$$

Mit den aus Widerstands- und Suszeptibilitätsmessungen bestimmten Übergangsfeldern

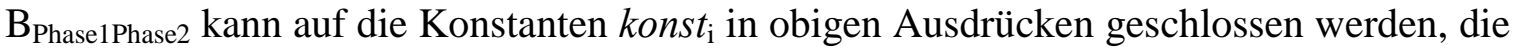
wiederum über Gl.4.1 den Betrag der Magnetisierung in den einzelnen Phasen bestimmen. Winzer et al. [Win99b] erhalten so für die Magnetisierung in den metamagnetischen Phasen $\mathrm{M}_{\mathrm{M} 1}=(1 / 4)(1 / \sqrt{ } 2) \mathrm{M}_{\mathrm{sat}}=(1 / 4) \mathrm{M}_{3}$ und $\mathrm{M}_{\mathrm{M} 2}=(1 / 4) \mathrm{M}_{\mathrm{sat}}=(1 / 4) \mathrm{M}_{\mathrm{FM}}$. Diese finden sich zumindest für B || [110] auch in den bislang durchgeführten Magnetisierungsmessungen [Can97a], [Nau98] wieder.
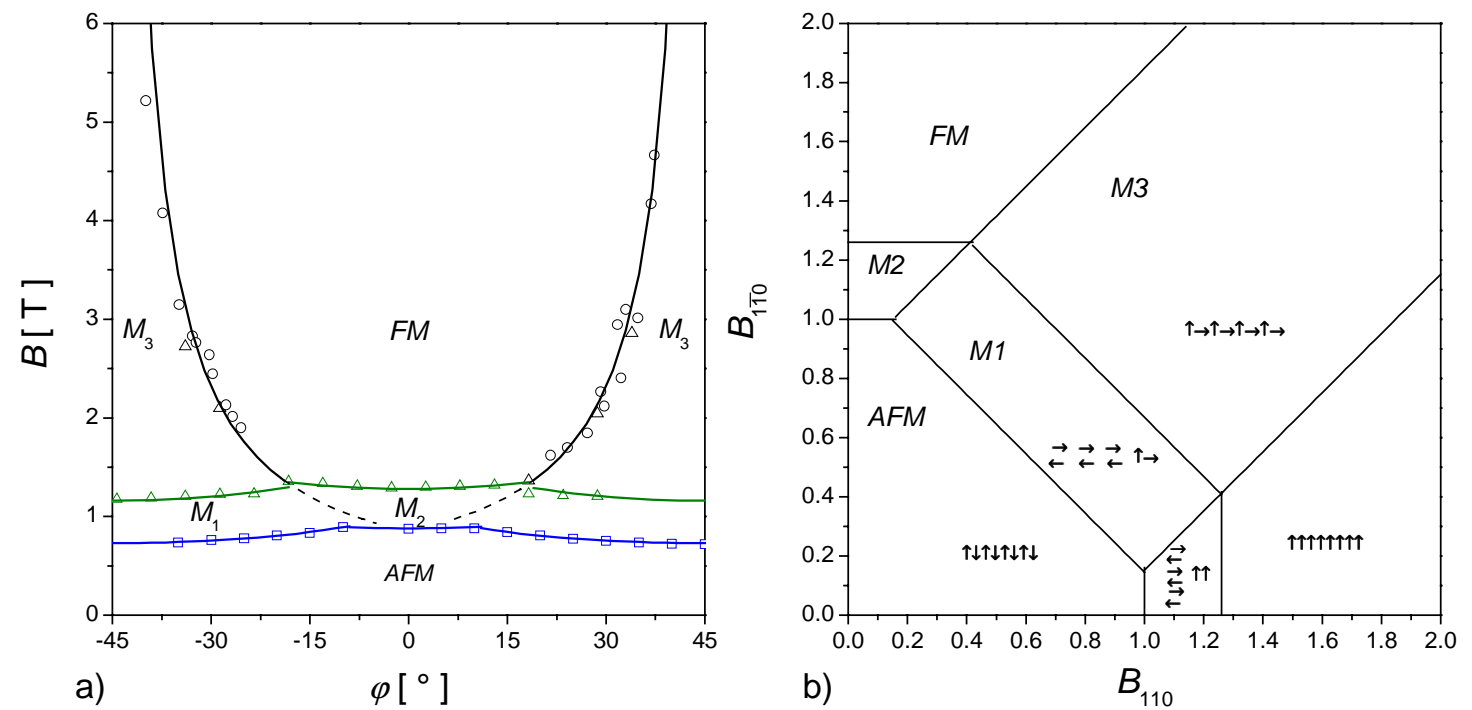

Abb.4.4 Magnetisches Phasendiagramm für $T=1.4 \mathrm{~K}$ nach [Win99b]. In a) sind die experimentellen (Symbole) und berechneten (Linien) anisotropen Phasengrenzen wie üblich als $B_{\text {grenz }}(\varphi)$ dargestellt. In b) die berechneten Phasengrenzen aus a) in einer ([110],[1 10$])$-Ebene.

Abb.4.4b zeigt das in dieser Weise für $T=1.4 \mathrm{~K}$ bestimmte Phasendiagramm in der ([110],[1 $\overline{1} 0])$-Ebene. Dabei stehen Pfeile der Art $\uparrow$ für Ebenen magnetischer Momente entlang der leichten Richtung [110] und Pfeile der Art $\rightarrow$ für eben solche mit Momentrichtung entlang [1 $\overline{1} 0]$. Die resultierende Anzahl magnetischer Momente ohne antiparallelen Gegenpart ergibt damit in jeder Phase die oben erläuterten Magnetisierungsrichtungen und Beträge. Diese Einschränkung der magnetischen Momente auf in sich $\mathrm{fm}$ ausgerichteten $(a, b)$-Ebenen und variierender Kopplung entlang der $c$-Richtung 
wird in Anlehnung an Rechnungen von Kalatsky et al. [Kal98] zu den magnetischen Phasen im verwandten $\mathrm{HoNi}_{2} \mathrm{~B}_{2} \mathrm{C}$ auch als ,4-clock“-Modell bezeichnet.

Die Beschreibung beinhaltet dabei die Bildung der feldinduzierten Phasen in eindomänigen Strukturen. Dabei wird aus einem evtl. zweidomänigen Grundzustand 5 , mit magnetischen Momenten in den Domänen entlang $\pm[110]$ bzw. $\pm[1 \overline{1} 0]$, beim Erzeugen des ersten feldinduzierten Zustandes ein Eindomänenzustand gebildet.

In den bislang durchgeführten Magnetisierungsmessungen [Can97a], [Nau98] wurde immer von der parallel zum Magnetfeld gemessenen Magnetisierung auf die vorliegende Struktur und den mit ihr verknüpften Magnetisierungsanteil senkrecht zum Magnetfeld geschlossen. Die im folgenden Kap 4.2.1 erläuterte Cantilevermeßmethode erlaubt dagegen Rückschlüsse auf die magnetische Struktur durch Messung einer senkrecht zum Magnetfeld weisenden Komponente der Magnetisierung. Ziel der Experimente ist eine erweiterte Charakterisierung der bislang nur oberhalb $T=2.0 \mathrm{~K}$ bekannten Magnetisierung in $\mathrm{DyNi}_{2} \mathrm{~B}_{2} \mathrm{C}$.

Die hier gezeigten ersten Ergebnisse aus Neutronenstreuexperimenten an $\mathrm{DyNi}_{2} \mathrm{~B}_{2} \mathrm{C}-\mathrm{EK}$ wurden in Kooperation mit Herrn A. Kreyssig vom Institut für Angewandte Physik Dresden ermittelt. Sie stellen ein erstes, aber wichtiges Ergebnis zur Interpretation der mit dem Cantileververfahren gewonnen Ergebnisse dar. Eine umfangreichere Präsentation und Diskussion der Neutronenstreuergebnisse findet sich erst in [Kre00].

\subsection{Meßmethoden}

\subsubsection{Cantileverexperimente}

Die gezeigten Cantileverexperimente erfolgen bei Temperaturen $T \approx 0.4 \mathrm{~K}$ und homogenen Magnetfeldern $B \leq 4$ T. Die tiefen Probentemperaturen wurden wie bei den dHvAExperimenten durch ein zweistufiges Kühlverfahren erreicht. Als Vorkühlstufe und Träger des supraleitenden Magneten dient ein ${ }^{4} \mathrm{He}$-Badkryostat, in den ein sogenannter „toploading“ ${ }^{3} \mathrm{He}$-Einsat ${ }^{6}$ der Firma Oxford Instruments mit der Meßsonde eingeführt werden kann. Die Erzeugung tiefster Probentemperaturen $T \geq 0.28 \mathrm{~K}$ gelingt auch hier

\footnotetext{
${ }^{5}$ Verallgemeinert ist natürlich auch ein $n$-domäniger $\mathrm{afm}$ Grundzustand möglich. Zur vereinfachten Diskussion genügt es hier $n=2$ zu betrachten.

${ }^{6}$ Interne Firmenbezeichnung: Heliox ${ }^{\mathrm{VL}}$
} 
durch Dampfdruckerniedrigung eines ${ }^{3} \mathrm{He}$-Bades, der primären Kühlstufe. Diese wird zur Erzeugung von Temperaturen $T_{{ }^{3} \mathrm{He}} \leq T_{{ }^{4} \mathrm{He}}$ durch ein Isolationsvakuum thermisch vom umgebenden ${ }^{4} \mathrm{He}$ abgekoppelt. Die Effizienz der hier verwendeten Anordnung ist durch die Verwendung einer integrierten Sorbtionspumpe mit sehr kurzem Pumpweg $(\approx 20 \mathrm{~cm})$ zum ${ }^{3} \mathrm{He}$-Bad gegenüber herkömmlichen Lösungen mit externen mechanischen Pumpen um ein Vielfaches gesteigert. Mit dem hier verwendeten Einsatz und der Cantilevermeßsonde können Probentemperaturen $T \leq 0.4 \mathrm{~K}$ über mehrere Stunden konstant gehalten werden.

Die Abb.4.5 und Abb.4.6zeigen Schemazeichnungen der verwendeten Meßsonde, die auf dem Cantileverprinzip basiert. Für die Biegung eines Cantilevers der Dicke $2 c$, das an einem Ende $x=0$ durch die Kraft $F$ belastet wird, gilt für die Auslenkung $v(x)$ am Ort $x$ (siehe bspw. [Tim51]):

Gl.4.3 $\quad v(x)=\frac{1}{6} \frac{\tau x^{3}}{E J l}-\frac{1}{2} \frac{\tau l x}{E J}+\frac{1}{3} \frac{\tau l^{2}}{E J}$

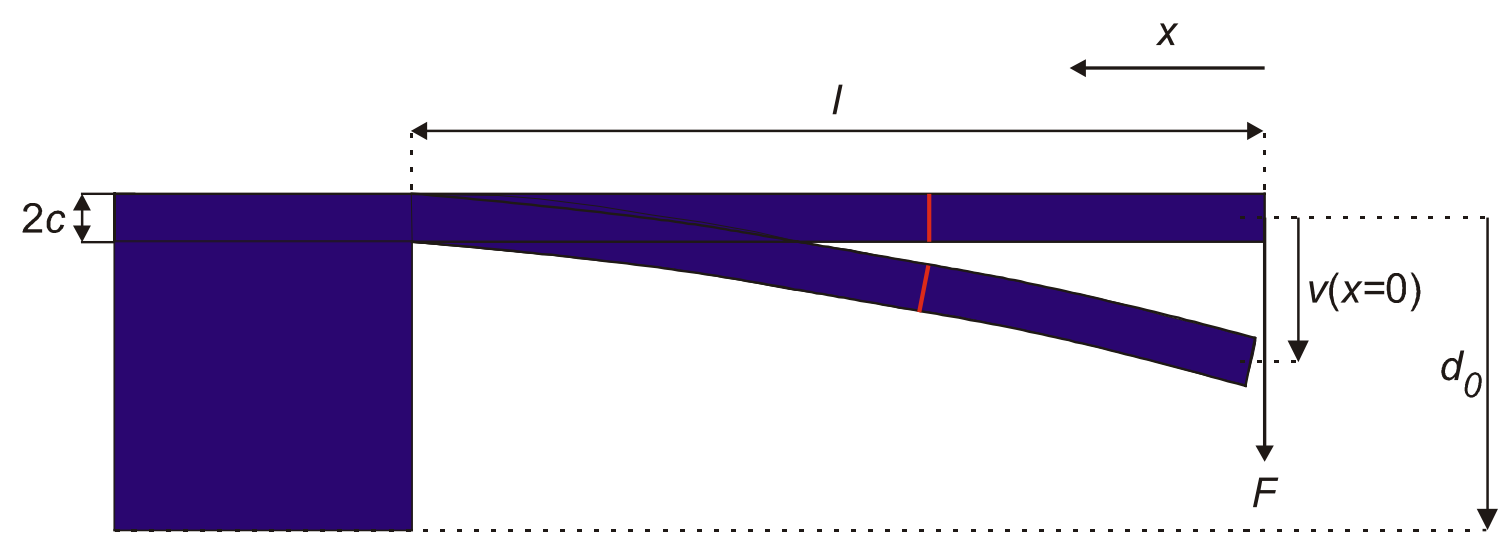

Abb.4.5 Schemazeichnung zur Drehmomentabhängigkeit der Cantileverbiegung.

Die Durchbiegung $v(x=0)$, welche von der ungescherten neutralen Faser gezählt wird, ist am Ende $x=0$ also direkt proportional zum angreifenden Drehmoment $\tau=l \cdot F$ :

Gl.4.4 $\quad v(x=0)=\frac{1}{3} \frac{\tau l^{2}}{E J}$. 
Die Proportionalitätskonstanten sind die Länge des Cantilevers $l$, das Elastizitätsmodul $E$ des Cantilevermaterials (hier: Silizium) für Längenänderung entlang $x$ und das Flächenträgheitsmoment $J=2 / 3 m_{\text {Fläche }} c^{2}$ einer im Schnitt der Abb.4.5 rot hervorgehobenen Einheitsfläche bei der durch die Verbiegung hervorgerufenen Rotation.

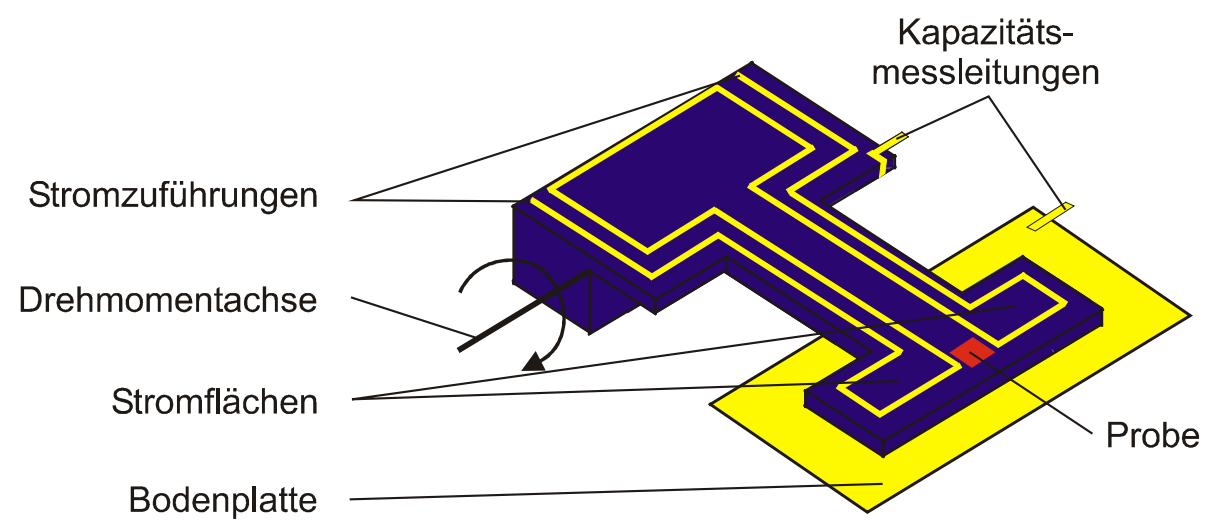

Abb.4.6 Schemazeichnung des hier verwendeten Catilevermagnetometers.

Die drehmomentabhängige Abstandsänderung $v(x)$ wird bei der hier verwendeten Cantileversonde ${ }^{\square}$ kapazitiv gemessen. D.h. der von unten goldbeschichtete bewegliche Cantileverarm bildet zusammen mit einer festen Gegenelektrode (goldbeschichtete Bodenplatte in Abb.4.6 einen Meßkondensator, dessen Kapzität $C$ nach einem Wechselstromverfahren mit einer Kapazitätsmeßbrücke bestimmt wird. Das gemessene Kapazitätssignal ist eine Funktion der Abstandsänderung $\Delta d \approx v(x=0)$ des Cantileverarms relativ zur Gegenelektrode:

Gl.4.5 $\quad C=C_{0} \cdot \frac{1}{1+\alpha \frac{\Delta d}{d_{0}}}$

Dabei ist der Plattenabstand $d_{0}$ für unbelastetes Cantilever beim hier verwendeten Meßsystem von der Größenordnung $0.1 \mathrm{~mm}$ und die Nullkapazität ohne wirkendes Drehmoment beträgt $C_{0}=0.5343 \mathrm{pF}$. Die Konstante $\alpha$ berücksichtigt, daß die Kapazitätsänderung nicht an einer lokalen Stelle $x=0$ anfällt, sondern daß das Kondensatorsystem entlang der $x$-Richtung ausgedehnt ist.

\footnotetext{
${ }^{7}$ Interner Firmenname der Firma Oxford Instruments $C M P:=$ Cantilever Magnetometer Probe
} 
Die signalerzeugenden Drehmomente sind hier magnetischer Natur $\tau=M \cdot B \sin (\delta)$, wobei $\delta$ den Winkel zwischen der Probenmagnetisierung und der Magnetfeldrichtung beschreibt $\delta \angle(M, B)$. Zur Charakterisierung anisotroper Probenmagnetisierungen muß die Orientierung der Probe relativ zu $B$ variabel sein. Dies ist durch eine Rotation des gesamten Cantilevermagnetometers um eine Achse parallel zur Drehmomentachse realisiert bei konstanter äußerer Magnetfeldrichtung (siehe Abb.4.7. Mit $\Delta d \approx v(x=0) \propto \tau=M B \sin (\delta)$ ergibt sich aus Gl.4.5 die Abhängigkeit der Meßkapazität bzw. ihrer Änderung relativ zu $C_{0}$ von einem magnetisch erzeugten Drehmoment.

Gl.4.6

$$
C=C_{0} \cdot \frac{1}{1+\beta \cdot M B \sin (\delta)}
$$

$$
\Rightarrow \Delta C=C-C_{0}=-C_{0} \cdot \frac{\beta \cdot M B \sin (\delta)}{1+\beta \cdot M B \sin (\delta)}
$$

Dabei ist $\beta=\alpha \frac{1}{3} \frac{l^{2}}{E J}$ der Zusammenschluß der Konstanten aus G1.4.4 und GI.4.5.

Aus Eichmessungen mit konstantem magnetischen Moment mittels definiertem Stromfluß durch die auf das Cantilever aufgebrachten Stromschleifen (siehe Abb.4.6 und definiertem Magnetfeld $B$ erhält man die Proportionalitätskonstante $\beta=5.032 \cdot 10^{+5} \mathrm{~J}^{-1}$.

Zur Bestimmung der magnetischen Phasen in $\mathrm{DyNi}_{2} \mathrm{~B}_{2} \mathrm{C}$ für $B$ parallel zur ([110],c)Ebene bzw. B parallel zur ([100],c)-Ebene wurde ein plättchenhafter Einkristall direkt auf dem Cantilever plaziert (siehe Abb.4.6). Hierbei liegen die kristallographischen [110]bzw. [100]-Richtungen parallel zur Cantileverachse $\left( \pm 1\right.$ bis $\left.2^{\circ}\right)$, d.h. die Drehmomentachse weist entlang der [1 $\overline{1} 0]$ - bzw. [010]-Probenachse. Zur Fixierung der Proben diente eine bei tiefen Temperaturen aushärtende dünne Fettschicht zwischen Probe und Cantilever.

Für Experimente $B$ parallel zur $(a, b)$-Ebene eines $\mathrm{DyNi}_{2} \mathrm{~B}_{2} \mathrm{C}$-Einkristalls ist ein direktes Fixieren auf dem Cantilever nicht möglich, da die geringe Kontaktfläche der plättchenhaften Proben $\left(A_{\mathrm{ac}} \leq 0.1 \mathrm{~mm}^{2}\right)$ in der $(a, c)$-Ebene nicht ausreicht, diese stabil zu positionieren. Daher wurden für diese Experimente einkristalline Plättchen mit kreisförmiger Querschnittsfläche und Massen $m_{\text {Probe }} \leq 100 \mu \mathrm{g}$ auf kleine Siliziumstäbe geklebt. Die 
Kontaktfläche $A_{\mathrm{Si}}=\left(l_{\mathrm{Si}} \cdot b_{\mathrm{Si}}\right) \approx 2 \cdot 1 \mathrm{~mm}^{2}$ ist über die aushärtende Fettschicht ausreichend, um die Probe mit dem Si-Stäbchen auf dem Cantilever zu fixieren (siehe Abb.4.7). Ein Nachteil dieses experimentell notwendigen Vorgehens ist die Veränderung der in Gl.4.4 eingehenden Parameter zur Berechnung der Biegungslinie. Neben der Verkürzung der Biegelänge des Cantilevers $l$ werden auf der Länge $l_{\mathrm{Si}}$ das effektive Elastizitätsmodul $E$ und das Flächenträgheitsmoment $J$ erhöht. Dies entspricht einer Verringerung der Konstanten $\beta$ aufgrund obiger Modifikationen der

a)

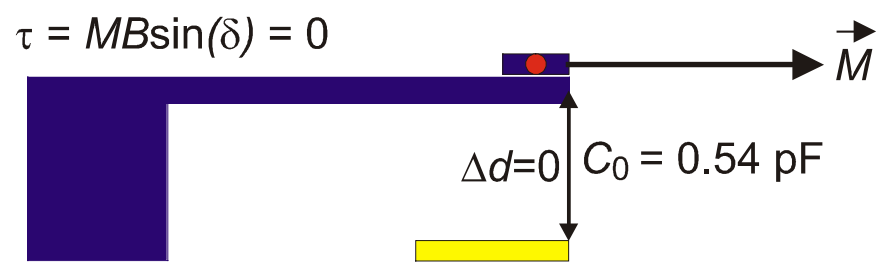

b)

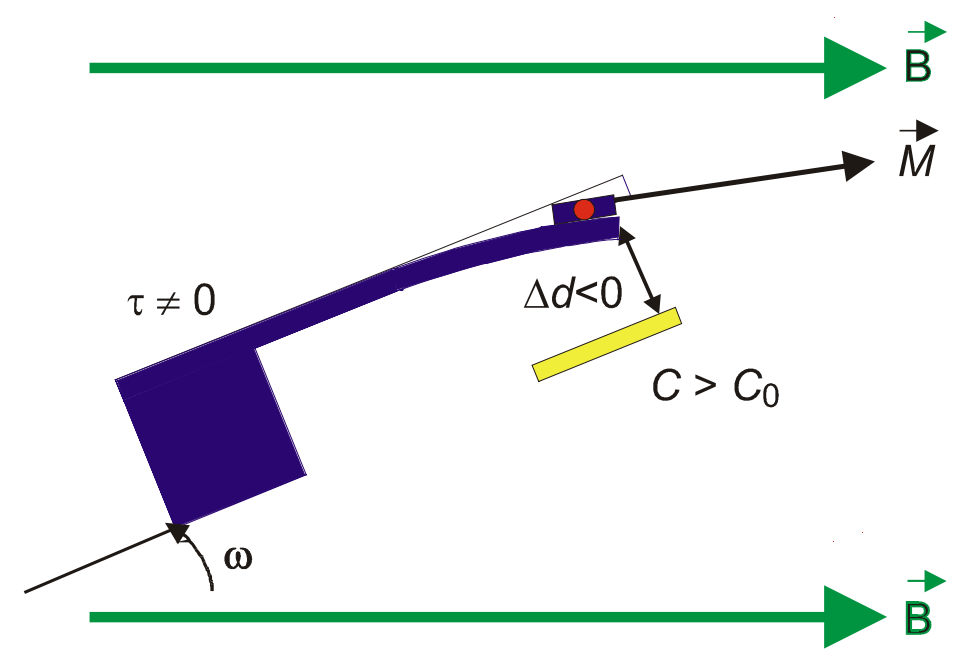

Abb.4.7 Konfigurationsskizze für Cantilevermessungen mit B $\|(a, b)$-Ebene. Bei a) liegt die Probenmagnetisierung parallel zum Feld, d.h. $\tau=0$. In b) ist die gesamte Cantilevereinheit um den Winkel $\omega$ relativ zur Position in a) verkippt und $\delta \angle(B, M) \neq 0$.

Cantilevereigenschaften. Da $\beta$ für diese Experimente nicht genau bekannt ist, kann keine absolute Bestimmung der Magnetisierung erfolgen. Allerdings erhält man Aussagen über die Magnetisierungsrichtung. So folgt aus Gl.4.6, daß speziell ein Vorzeichenwechsel in $\Delta C$ einem Vorzeichenwechsel des wirkenden Drehmomentes $M B \sin (\delta)$ entspricht. 
Der für die Cantileverexperimente innerhalb der ([110],c)- bzw. der ([100],c)-Ebene verwendete Einkristall hat eine Masse von $220 \mu \mathrm{g}$. Die durch die Sättigungsmagnetisierung der $\mathrm{Dy}^{3+}$-Momente $\mu_{\text {sat }}=9.7 \mu_{\mathrm{B}}$ hervorgerufene Sättigungsmagnetisierung $M_{\mathrm{sat}}$ der Probe berechnet sich aus dem Verhältnis der Probenmasse und der Masse einer Formeleinheit $\mathrm{DyNi}_{2} \mathrm{~B}_{2} \mathrm{C}$ zu $M_{\text {sat }}=3.8 \cdot 10^{-5} \mathrm{~J} / \mathrm{T}$. Die Magnetisierungsrichtung liegt aufgrund der Kristallfeldaufspaltung am SE-Platz innerhalb der $(a, b)$-Ebene des Systems [Kal98]. Bei Angabe des Winkels $v$ zwischen der $(a, b)$-Ebene des Einkristalls und der Magnetfeldrichtung $B$ kann der Anteil der Probenmagnetisierung $M$ innerhalb der $(a, b)$-Ebene relativ zu ihrer Sättigungsmagnetisierung bestimmt werden. Mit $M=x \cdot M_{\text {sat }}$ erhält man aus G1.4.6:

$$
\begin{gathered}
\text { Gl.4.7 } \Delta C=C-C_{0}=-C_{0} \cdot \frac{\beta\left(x \cdot M_{s a t} B \sin (v)\right)}{1+\beta\left(x \cdot M_{s a t} B \sin (v)\right)} \quad \text { und } \\
x=\frac{\Delta C}{\Delta C-C_{0}} \cdot \frac{1}{\beta\left(M_{\text {sat }} B \sin (v)\right)} .
\end{gathered}
$$

Aufgrund der Probenmagnetisierung in der Größenordung $M_{\text {sat }} \approx 10^{-5} \mathrm{~J} / \mathrm{T}$ mußten die Experimente mit $B \|[110]$ und $B \|$ [100] bei kleinen Winkeln $v$ erfolgen, um zu gewährleisten, daß das Cantilever nicht aufsetzt, d.h $\Delta d \leq d_{0}$. Mit Gl.4.7 folgt daraus die Konsequenz eines großen Fehlers $\Delta x / x$. Die in Kap.4.3 angegebenen Magnetisierungsverhältnisse ergeben daher nur Tendenzen für die vorliegende Probenmagnetisierung.

Alle Experimente wurden als Funktion eines sich schrittweise ändernden Magnetfeldes bestimmt. Die Schrittweite der Magnetfeldstützstellen lag bei $25 \mathrm{mT}$, wobei für jeden Feldwert 40 Meßpunkte ermittelt wurden. Für die dargestellten Meßkurven ist $\Delta C(B)$ für jeden Feldwert der Mittelwert aus den 40 Meßwerten.

\subsubsection{Neutronenstreuung}

Zur erweiterten Untersuchung der metamagnetischen Zwischenphasen in $\mathrm{DyNi}_{2} \mathrm{~B}_{2} \mathrm{C}$ wurden erste elastische Neutronenstreuexperimente im angelegten Magnetfeld an $\mathrm{DyNi}_{2} \mathrm{~B}_{2} \mathrm{C}$-EK durchgeführt. Die Prinzipien der Neutronenstreuung sind eng mit den anderen zur Festkörpercharakterisierung eingesetzten Beugungsmethoden wie Elektro- 
nen- oder Röntgenbeugung verknüpft (siehe beispw. [Fur95]). Die Grundvoraussetzung zur Charakterisierung von Festkörperperiodizitäten auf Längenskalen bis hinab zu einigen Angström ist sowohl für die de Broglie Wellenlänge thermischer Neutronen als auch Röntgenstrahlung erfüllt. Man erhält aus den Strukturfaktoren $F(\vec{q})$, zu deren Betragsquadrat die unter dem Streuvektor $\vec{q}$ gemessene Intensität proportional ist, in Streuexperimenten beider Art Aussagen über die Kristallstruktur des Probengitters. Speziell für Neutronenstreuung gilt :

Gl.4.8 $\quad I_{\text {Kern }}(\vec{q}) \propto\left|F_{\text {Kern }}(\vec{q})\right|^{2}=\left|\sum_{j} b_{\mathrm{j}} \exp \left[2 \pi i\left(\vec{q} \cdot \vec{r}_{\mathrm{j}}\right)\right] \cdot T_{\mathrm{j}}(\vec{q})\right|$

Dabei ergibt sich der Strukturfaktor der Kernstreuung im elastischen Fall zur Fouriertransformierten der Kerndichteverteilung des vorliegenden Probengitters, gewichtet nach den Streulängen $b_{\mathrm{j}}$ der Atomkerne der Probe. $T_{\mathrm{j}}(\vec{q})$ steht für den Debye-Waller-Faktor, der die Nullpunktsschwingung der Probenatome um ihre Gleichgewichtspositionen berücksichtigt [Kit88]. Man erhält daher nur für Streuvektoren $\vec{q}=\vec{k}_{\text {gestreut }}-\vec{k}_{\text {einfallend }}$ nennenswerte Intensität, die Elemente des reziproken Gitters $\vec{q}=h a^{*}+k b^{*}+l c^{*}$ der zu Grunde liegenden Kristallstruktur sind. Speziell für den Fall der Borkarbide mit Raumgruppe $I 4 / \mathrm{mmm}$ [Sie94] ergeben sich damit die Auswahlregeln ${ }^{8} \sum(h+k+l) \stackrel{!}{=} 2 n$ in der rechtwinkligen Basis $\vec{a}^{*}=\frac{2 \pi}{a} \vec{e}_{\mathrm{a}}, \vec{b}^{*}=\frac{2 \pi}{a} \vec{e}_{\mathrm{b}}$ und $\vec{c}^{*}=\frac{2 \pi}{c} \vec{e}_{\mathrm{c}}$.

Außer dem durch die Kern-WW der Neutronen begründeten gibt es einen zusätzlichen Streubeitrag $I_{\text {Mag }}(\vec{q})$ durch die Dipol-WW des magnetischen Moments des Neutrons $\left(\mu_{\text {Neutr }}=-1.913 \cdot \mu_{\text {Kern }}\right)$ mit den magnetischen Momenten im Festkörper. Der sich daraus ergebende magnetische Strukturfaktor $F_{\text {Mag }}(\vec{q})$ ist die Fouriertransformierte dieser Spindichteverteilung:

\footnotetext{
${ }^{8}$ Eine Ausnahme hinsichtlich dieser Auswahlregeln bildet der Kernreflex (002). Dieser tritt aufgrund einer zufälligen Auslöschung in $\mathrm{DyNi}_{2} \mathrm{~B}_{2} \mathrm{C}$ bei Neutronenstreuung nur mit extrem geringer Intensität auf [Kre99a].
} 
Gl.4.9 $\quad I_{\text {Mag }}(\vec{q}) \propto\left|F_{\text {Mag }}(\vec{q})\right|^{2}=\left|\sum_{j} b_{\mathrm{Mj}} \exp \left[2 \pi i\left(\vec{q} \cdot \vec{r}_{\mathrm{j}}\right)\right] \cdot T_{\mathrm{j}}(\vec{q})\right|$.

Dabei ist $T_{\mathrm{j}}(\vec{q})$ wiederum der Debye-Waller-Faktor. Die magnetische Streulänge $b_{\mathrm{Mj}}(\vec{q}) \propto f_{M j}(\vec{q}) \hat{\sigma} m_{\perp}(\vec{q})$ setzt sich zusammen aus dem magnetischen Formfaktor $f_{M j}(\vec{q})$, gleichbedeutend mit der Fouriertransformierten der normalisierten Magnetisierungsdichte des $j$-ten Atoms

$$
f_{\mathrm{Mj}}(\vec{q})=\int_{V} M_{\mathrm{j}}(\vec{r}) \exp [2 \pi i(\vec{q} \cdot \vec{r})] d \vec{r} \text { und } f_{\mathrm{Mj}}(0)=\int_{V} M_{\mathrm{j}}(\vec{r}) d \vec{r}=1,
$$

und dem Produkt des Neutronenspinoperators $\hat{\sigma}$ mit dem Anteil $m_{\perp}(\vec{q})$ des magnetischen Momentes des $j$-ten Atoms in der zu $\vec{q}=(h k l)$ gehörenden Streuebene.

Für die hier durchgeführten Experimente an $\mathrm{DyNi}_{2} \mathrm{~B}_{2} \mathrm{C}$ bedeutet dies, daß die magnetische Streulänge $b_{\mathrm{Mj}}(\vec{q})$ von der Projektion des magnetischen Moments jedes Dy-Atoms senkrecht zum untersuchten Streuvektor $\vec{q}$ bestimmt wird.

Die in obiger Form probenabhängigen Intensitäten der magnetischen Streuung $I_{\text {Mag }}(\vec{q})$ und der Streuung am Kern $I_{\text {Kern }}(\vec{q})$ sind von gleicher Größenordnung und addieren sich im Fall der hier angewandten Streuung unpolarisierter Neutronen:

Gl.4.10

$$
I(\vec{q})=I_{\mathrm{Mag}}(\vec{q})+I_{\mathrm{Kern}}(\vec{q})
$$

$$
=\left|F_{\mathrm{Mag}}(\vec{q})\right|^{2}+\left|F_{\mathrm{Kern}}(\vec{q})\right|^{2}
$$

Zur Charakterisierung der felderzeugten magnetischen Phasen wurden feldabhängige Streuintensitäten $I(\vec{q}, \vec{B})$ für die beiden Orientierungen $B \|[110]$ bzw. $B \|[100]$ bei $T=2.0 \mathrm{~K}$ mittels elastischer Neutronenstreuung in den Diffraktometern D15 bzw. D23 am Institut-Laue-Langevin in Grenoble bestimmt Abb.4.8 zeigt eine schematische Zeichnung des Meßaufbaus am Diffraktometer D15).

Die in Kap.4.3.2 gezeigten Intensitätsverläufe $I(\vec{q}, \vec{B})$ sind integrierte Intensitäten der Detektorzählrate für $\Omega$-Scans bei festem Braggwinkel $2 \Theta$ des jeweiligen Streuvektors $\vec{q}$ und quasi statisch variierendem Magnetfeld $\mathrm{d} B / \mathrm{d} t \approx 1 \mathrm{~T} / \mathrm{h}$. Da sich die zugrunde liegende 
Kristallstruktur bei den hier gezeigten Experimenten nicht ändert (vergleiche im Gegensatz dazu bspw. [Kre99]) sind Änderungen in der Streuintensität $I(\vec{q})$ als Funktion eines angelegten Magnetfeldes auf Änderungen des Beitrags $I_{\text {Mag }}(\vec{q})$ der Gl.4.10 zurückzuführen.

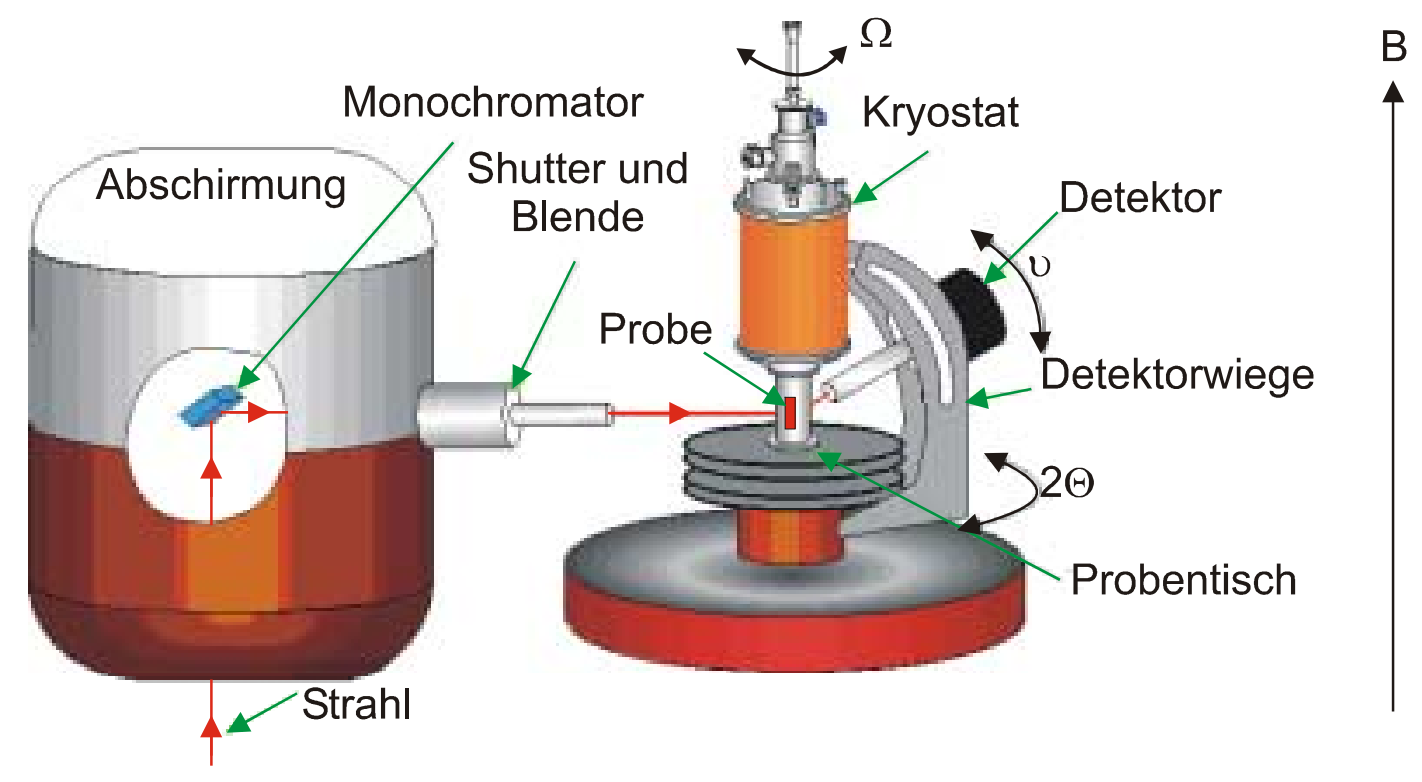

Abb.4.8 Schematischer Aufbau des Diffraktometers D15 am ILL in Grenoble. Nach [Bur96].

\subsection{Ergebnisse}

\subsubsection{Cantileverergebnisse}

Im folgenden werden die mit dem Cantileververfahren gewonnen Ergebnisse präsentiert. Die ersten Experimente behandeln dabei Messungen, bei denen der Einkristall direkt auf dem Cantilever liegt, also mit $B \|([110], c)$-Ebene bzw. $B \|([100], c)$-Ebene des EK. Die aufgetragene Kapazitätsänderung $\Delta C(B)$ wird damit durch Drehmomente entlang der [1 $1 \overline{1} 0]-$ bzw. [010]-Richtung hervorgerufen.

Bei den Experimenten mit $B \|(a, b)$-Ebene ist die Drehmomentachse parallel zur [001]Achse des Einkristalls. 


\subsubsection{Cantileverexperimente für $B \|([110], c)$-Ebene}

In Abb.4.9 sind zwei Meßverläufe des Kapazitätssignales $\Delta C(B)$ für die Orientierung $B \|[110]-3^{\circ}$ bei $T=0.4 \mathrm{~K}$ dargestellt. Man erkennt sowohl bei Felderhöhung (>) als auch bei Felderniedrigung $(<)$ mehrere stufenförmige Übergänge bei Feldern $B_{\mathrm{i}}$, deren Position durch gestrichelte vertikale Linien hervorgehoben ist. Oberhalb $B=2.0 \mathrm{~T}$ konnten keine signifikanten Signaländerungen beobachtet werden.

Für Magnetfelderhöhung ist knapp unterhalb $B_{\mathrm{FM}}$ ein schwacher zusätzlicher Übergang bei $B_{3}>\mathrm{zu}$ sehen, der für Felderniedrigung nicht beobachtet wird. Alle Übergangsfelder $B_{\mathrm{i}}$ zeigen ausgeprägte Hysterese, mit stärkster Ausbildung in $\Delta B_{1}=\left(B_{1>-}-B_{1<}\right) \leq 0.9 \mathrm{~T}$.

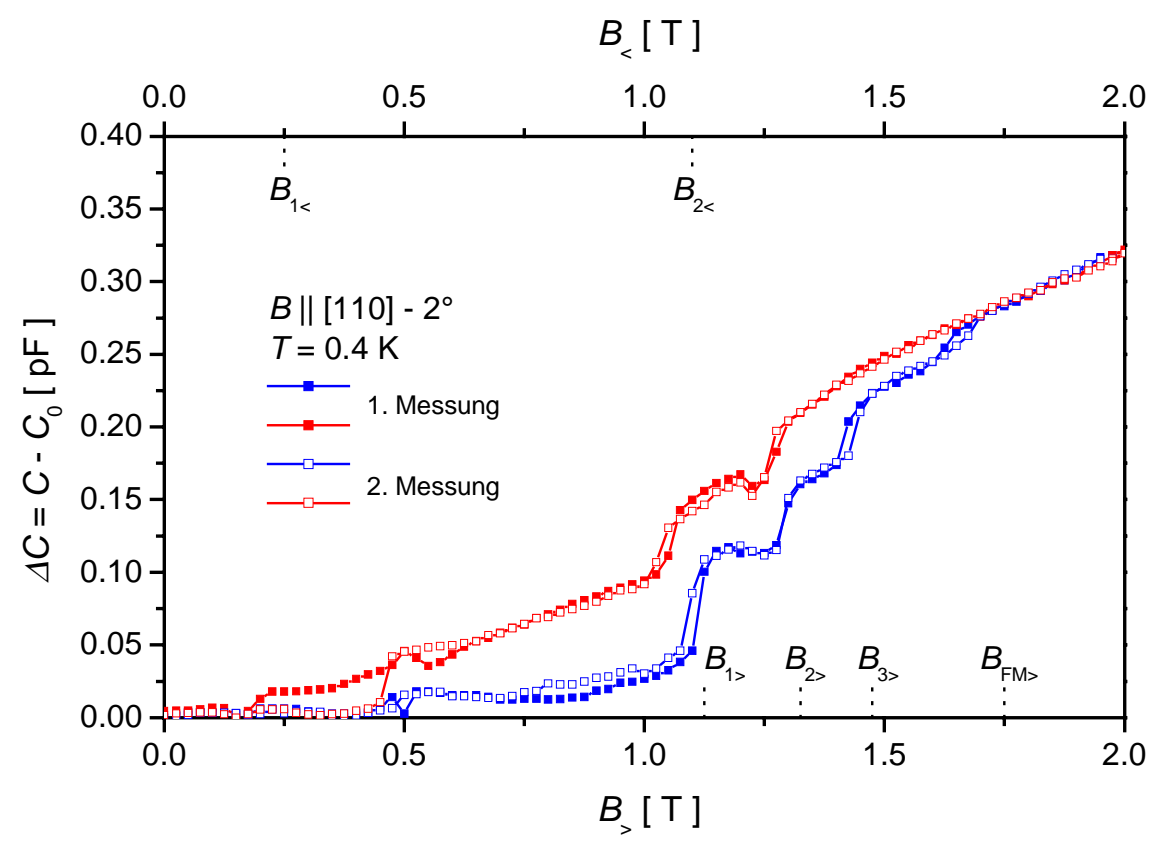

Abb.4.9 Kapazitätssignal $\Delta C$ für $T=0.4 \mathrm{~K}$ und B $\|([110], c)$-Ebene. Blau dargestellte Meßwerte wurden bei Felderhöhung, rot dargestellte bei Feldverminderung aufgenommen.

Die 2. Messung bei gleichen Meßbedingungen dokumentiert, daß die Größe der Hysterese in $\Delta B_{1}$ vom vorherigen Prozeßverlauf abhängt.

Mit $v=-2^{\circ}$ und $M_{\mathrm{sat}}=3.8 \cdot 10^{-5} \mathrm{~J} / \mathrm{T}$ erhält man aus Gl.4.7 eine Abschätzung über den Anteil $x$ der Sättigungsmagnetisierung in den felderzeugten magnetischen Phasen zwischen zwei $B_{\mathrm{i}}$. Für Felderhöhung gilt für die einzelnen Übergangsfelder $B_{\mathrm{i}}>$ :

$$
x\left(B_{1>}=1.125 \mathrm{~T}\right) \approx 0.34, \text { d.h. } M_{\text {Probe }}\left(B_{1>}\right) \approx 1 / 3 \cdot M_{\text {sat }}
$$




$$
\begin{aligned}
& x\left(B_{2>}=1.325 \mathrm{~T}\right) \approx 0.49, \text { d.h. } M_{\text {Probe }}\left(B_{2>}\right) \approx 1 / 2 \cdot M_{\text {sat }} \\
& x\left(B_{3>}=1.475 \mathrm{~T}\right) \approx 0.71, \text { d.h. } M_{\text {Probe }}\left(B_{3>}\right) \approx 3 / 4 \cdot M_{\text {sat }} \\
& x\left(B_{\mathrm{FM}>}=1.75\right) \approx 0.95, \text { d.h. } M_{\text {Probe }}\left(B_{\mathrm{FM}}\right) \approx 1 \cdot M_{\mathrm{sat}}
\end{aligned}
$$

Für die magnetischen Zwischenphasen bei Feldverminderung $(<)$ gilt entsprechend:

$$
\begin{aligned}
& x\left(B_{2<}=1.1 \mathrm{~T}\right) \approx 0.56, \text { d.h. } M_{\text {Probe }}\left(B_{2<}\right) \approx 1 / 2 \cdot M_{\mathrm{sat}} \\
& x\left(B_{1<}=0.25\right) \approx 0.23, \text { d.h } M_{\text {Probe }}\left(B_{1<}\right)<1 / 4 \cdot M_{\mathrm{sat}}
\end{aligned}
$$

Diese Werte $x$ ergeben allerdings nur Tendenzen für die Magnetisierung in den einzelnen Phasen, da sie aufgrund des klein zu wählenden $v=-2^{\circ}$ stark fehlerbehaftet sind (siehe Gl.4.7). Man kann ihnen aber ablesen, daß bis auf $B_{3}$ alle bei Felderhöhung beobachteten Zwischenzustände auch bei Felderniedrigung eingenommen werden.

\subsubsection{Cantileverexperimente für $B \|([100], c)$-Ebene}

In Abb. 4.10 sind zwei Meßverläufe des Kapazitätssignales $\Delta C(B)$ für die Orientierung $B \|[100]-1^{\circ}$ bei $T=0.4 \mathrm{~K}$ dargestellt. Man erkennt sowohl bei Felderhöhung als auch bei Felderniedrigung einen stufenförmigen Übergang bei Magnetfeldern $B_{1}$.

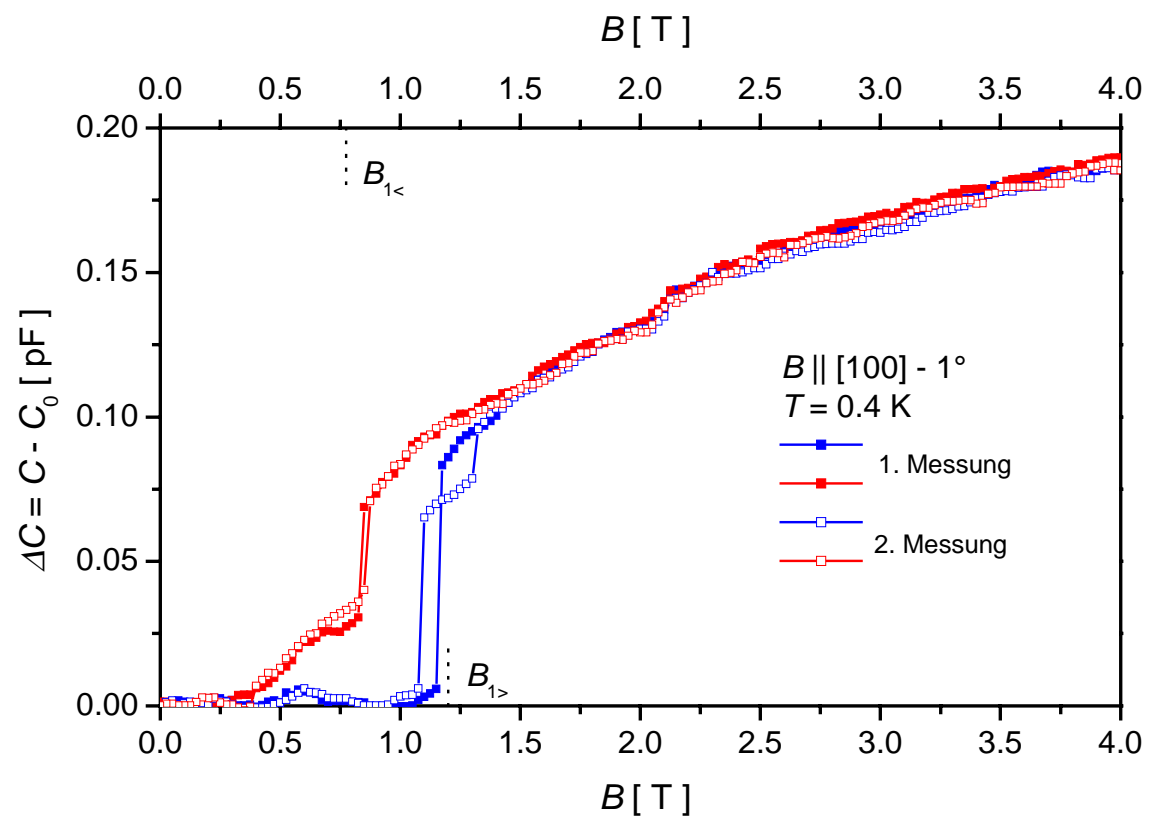

Abb. 4.10 Kapazitätssignal $\Delta C$ für $T=0.4 \mathrm{~K}$ und B $\|([100], c)$-Ebene. Blau dargestellte Meßwerte wurden bei Felderhöhung, rot dargestellte bei Feldverminderung aufgenommen. 
Der Übergang bei Felderhöhung spaltet bei wiederholter Prozeßführung in zwei Teilübergänge bei $B_{1>} \approx 1.1 \mathrm{~T}$ auf.

Mit $v=-1^{\circ}$ erhält man auch hier aus Gl.4.7 den Anteil der Sättigungsmagnetisierung für Magnetfelder $B>B_{2}$ > für Felderhöhung und für $B<B_{1<}$ bei Felderniedrigung:

$$
\begin{aligned}
& x\left(B_{2>}=1.5 \mathrm{~T}\right)=(1 / \sqrt{2}) \cdot 0.81, \text { d.h. } M_{\text {Probe }}\left(B_{2>}\right) \leq(1 / \sqrt{ } 2) \cdot M_{\text {sat }} \text { und } \\
& x\left(B_{1<}=0.825 \mathrm{~T}\right)=(1 / \sqrt{ } 2) \cdot 0.41 \text {, d.h. } M_{\text {Probe }}\left(B_{1<}\right) \leq 1 / 2 \cdot(1 / \sqrt{ } 2) \cdot M_{\text {sat }} \leq 1 / 3 \cdot M_{\text {sat }}
\end{aligned}
$$

Der vom Faktor $x$ beschriebene Anteil der Sättigungsmagnetisierung ist nur bestimmt von der Probenmagnetisierung senkrecht zur Drehmomentachse (siehe Abb.4.6. Für die hier durchgeführten Cantileverexperimente $B \|([100], c)$-Ebene sind dies also Probenmagnetisierungen entlang [100]. Bei der Bildung von felderzeugten magnetischen Zuständen durch effektive Magnetisierungen entlang der leichten $\langle 110\rangle$-Richtungen ist das hier gemessene Signal also nur durch deren Projektion auf die [100]-Richtung bestimmt. Dies begründet den Faktor $(1 / \sqrt{2})=\cos \left(45^{\circ}\right)$ zum Vergleich des gemessenen Magnetsisierungsanteils $x$ relativ zur Probenmagnetisierung. Damit erhält man für $B=1.5 \mathrm{~T}$ in etwa den aus anderen Magnetisierungsmessungen für diesen Feldbereich bestimmten Anteil $(1 / \sqrt{ } 2) \cdot M_{\text {sat }}[$ Nau98]. Wegen des klein zu wählenden $v$ können allerdings auch hier die angegebenen Magnetisierungswerte nur Tendenzen angeben. 


\subsubsection{Cantileverexperimente für $B \|(a, b)$-Ebene}
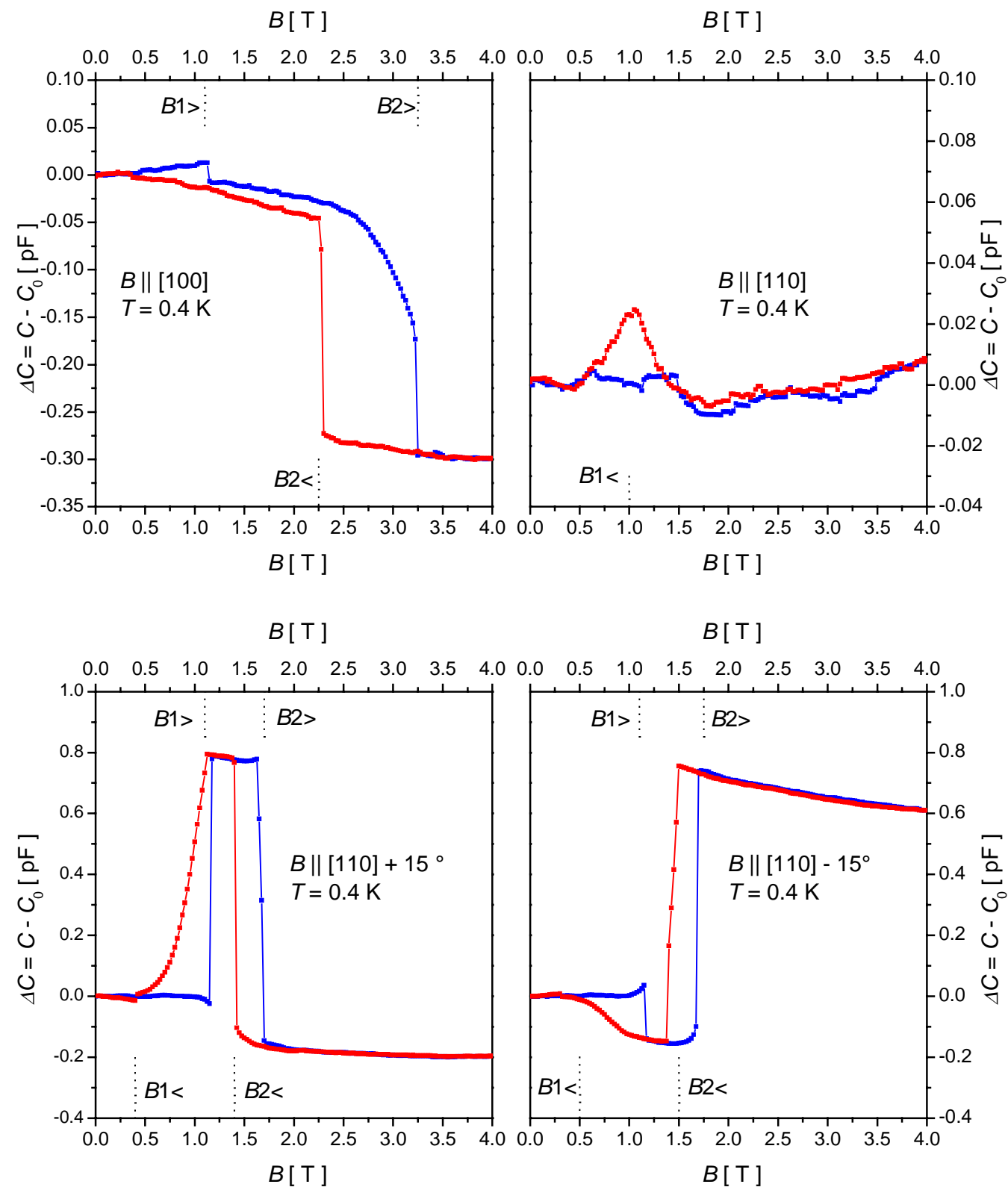

Abb.4.11 Repräsentative Meßkurven $\triangle C(B)$ für 4 ausgewählte Orientierungen $B \|(a, b)$-Ebene bei $T=0.4 \mathrm{~K}$. Blau dargestellte Meßwerte wurden bei Felderhöhung, rot dargestellte bei Feldverminderung aufgenommen.

Abb.4.11 zeigt vier repräsentative Meßkurven des Cantileversignales $B \|(a, b)$-Ebene bei $T=0.4 \mathrm{~K}$. Abgesehen von der Orientierung $B \|[110]$ beobachtet man für alle Orientierungen zwei Übergänge des Signals $\Delta C(B)$. Die felderzeugten Phasen bei $B_{1}$ bzw. $B_{2}$ 
bewirken umgekehrte Vorzeichen in $\Delta C(B)$. Nach den Ausführungen in Kap.4.2.1 erfolgt demnach für alle Orientierungen außer $B \|[110]$ eine Änderung der Magnetisierungsrichtung beim Übergang vom magnetischen Zustand für Magnetfelder $B_{1}<B<B_{2}$ zum Zustand oberhalb $B_{2}$. Da für keine der Orientierung zwei Übergange mit gleichem Vorzeichen der Signaländerung $\Delta C(B)$ beobachtet werden, gibt es in keiner Orientierung außer evtl. $B \|[110]$ eine Abfolge von felderzeugten Phasen, die gleiche Magnetisierungsrichtungen aufweisen. Der Übergang $B_{2}$ erfolgt bei Orientierungen nahe $B \|$ [110] bei tieferen Feldern als bei Orientierungen nahe $B \|$ [100], während der Übergang $B_{1}$ für Felderhöhung in allen Orientierungen nahezu konstant bei $B_{1}=1.1 \mathrm{~T}$ liegt. Aus dem verschwindenden Signal für $B \|[110]$ (man vergleiche die um den Faktor 10 gedehnte Skala verglichen mit den beiden symmetrischen Richtungen um $B$ \| [110]) kann geschlossen werden, daß die Magnetisierung in dieser Richtung für alle Magnetfelder oberhalb des afm Zustandes in [110]-Richtung weist. Aus den Vorzeichen der Signale $\Delta C\left(B_{1}\right)$ und $\Delta C\left(B_{2}\right)$ läßt sich auf die Magnetisierungsrichtung in den felderzeugten Phasen schließen.

Abb.4.12 stellt die Signalverläufe $\Delta C\left(B_{1}\right)$ und $\Delta C\left(B_{2}\right)$ als Funktion des Winkels $\varphi$ dar. Man sieht die bereits angesprochene Vorzeichenumkehr der Signale für festen Winkel und eine Symmetrie ihrer Nulldurchgänge bei $\varphi=0^{\circ} \pm n \cdot 45^{\circ}$. Betrachtet man eine Magnetisierung $M_{110}$ entlang der leichten [110]-Richtung der Proben, so erzeugt diese gemäß Gl.4.6 ein Signal, daß für Winkel $-45^{\circ}<\varphi<0^{\circ}$ positiv und für Winkel $0^{\circ}<\varphi<45^{\circ}$ negativ ist (siehe Abb. 4.13. Diese Winkelabhängigkeit wird gerade vom Signal $\triangle C\left(B_{2}\right)$ der magnetischen Phase für $B>B_{2}$ erfüllt. Entsprechend gilt die Übereinstimmung im Winkelbereich $|\varphi|>45^{\circ}$, wenn man statt der Magnetisierungsrichtung $M_{110}$ die kristallographisch und magnetisch äquivalenten Magnetisierungsrichtungen $M_{1-10}$ oder $M_{-110}$ betrachtet. Für diese gilt ebenfalls obige Argumentation, nur daß dann der Winkel $\varepsilon=\varphi \pm 90^{\circ} \mathrm{mit}|\varphi|<45^{\circ}$ zu berücksichtigen ist. 


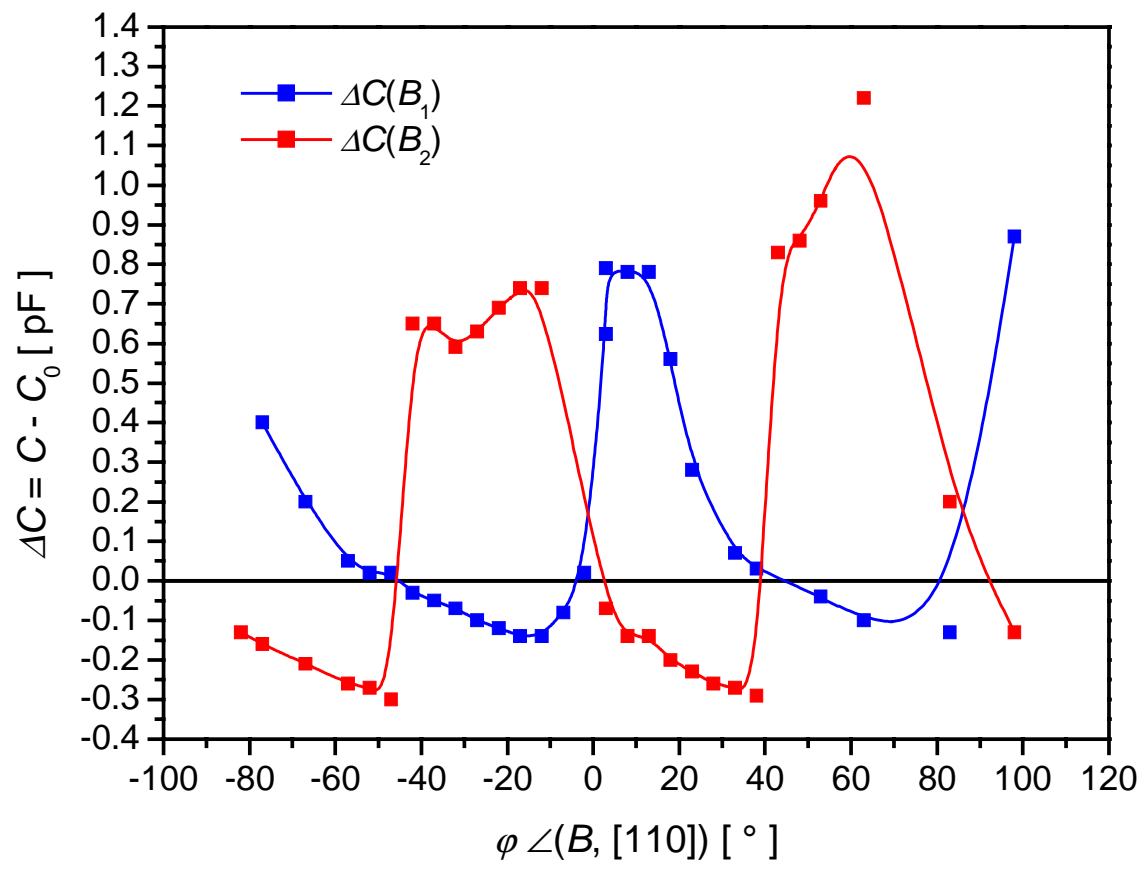

Abb.4.12 Winkelabhängigkeit des Signalverlaufes $\Delta C\left(B_{1}\right)$ und $\Delta C\left(B_{2}\right)$ aus Meßkurven anlog $4 b b .4 .11$.

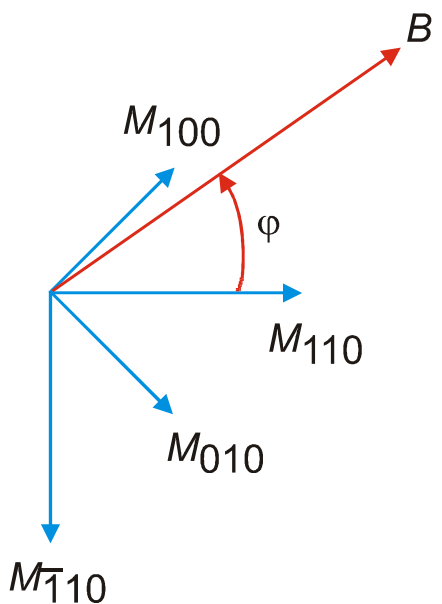

Abb. 4.13 Geometrie zu den im Text beschriebenen Überlegungen zur Signalerzeugung $\Delta C(\varphi)$ durch Probenmagnetisierungen $M_{110}$ und $M_{100}$.

Damit kann das oberhalb von $B_{2}$ erzeugte Kapazitätssignal $\Delta C\left(B_{2}\right)$ auf eine signalbestimmende Probenmagnetisierungen entlang der [110]-Richtung zurückgeführt werden. Die Magnetisierungsrichtung für Magnetfelder $B_{1}<B<B_{2}$ folgt zwangsläufig aus dem umgekehrten Vorzeichen des Signales $\Delta C\left(B_{1}\right)$ verglichen mit $\Delta C\left(B_{2}\right) . \Delta C\left(B_{1}\right)$ zeigt als Funktion des Winkels $\varphi$ eine um $45^{\circ}$ verschobenene Vorzeichenabhängigkeit relativ zu 
$\Delta C\left(B_{2}\right)$. Diese muß durch eine Magnetisierungsrichtung um $45^{\circ}$ verdreht zu [110] erzeugt werden, also parallel [100]. Damit liegt im gesamten untersuchten Winkelbereich bei Magnetfeldern $B_{1}<B<B_{2}$ eine magnetische Phase mit signalbestimmender [100]Magnetisierungsrichtung vor. Eine Ausnahme bildet dabei nur die Orientierung $B \|$ [110]. Aus den Cantileverexperimenten mit $B \|(a, b)$-Ebene folgt für diese Orientierung $\Delta C(B) \approx 0$, d.h die Magnetisierungsrichtung ist für jeden Feldwert parallel zu $B$. Aus dem steilen Anstieg in $\triangle C\left(B_{1}\right)$ um die Orientierung $B \|[110]$ (siehe Abb.4.12) kann geschlossen werden, daß diese Magnetisierungsrichtung $M_{110}$ nur für einen kleinen Winkelbereich $|\varphi|<5^{\circ}$ für Magnetfelder $B_{1}<B<B_{2}$ existiert.

Zusammenfassend ergibt sich das in Abb.4.14 dargestellte magnetische Phasendiagramm für $\mathrm{DyNi}_{2} \mathrm{~B}_{2} \mathrm{C}$ bei Magnetfelderhöhung für die Temperatur $T=0.4 \mathrm{~K}$. Die hier bestimmten Phasen $M_{100}$ bzw. $M_{010}$ unterhalb $B_{2}(\varphi)$ bestätigen über weite Teile des Winkelbereichs die von Winzer et al. [Win99b] unterhalb $B_{\mathrm{M} 3 \mathrm{FM}}$ vorhergesagten Magnetisierungsrichtungen für $T=1.4 \mathrm{~K}$ (siehe Abb.4.15). Unterschiedlich ist, daß außer der afm Phase bei Feldern unterhalb $B_{1}(\varphi) \approx 1.2 \mathrm{~T}$ und den Phasen mit Magnetisierungsrichtung entlang $<110>$-Richtungen oberhalb $B_{2}(\varphi)$ nur eine magnetische Zwischenphase beobachtet wird. Dies ist zumindest für $B \|[100]$ im Einklang mit dem Phasendiagramm nach Peng (siehe Abb.4.1, wonach bei tiefen Temperaturen die Phase $M_{1}$ nicht mehr beobachtet wird. $\mathrm{Ob}$ im Winkelbereich um $B \|[110]$ eine magnetische Phase $M_{2}$ mit $M \|$ [110] existiert, kann hier nicht sicher geschlossen werden.

Erstaunlich ist die sehr viel flacher verlaufende Winkelabhängigkeit von $B_{2}(\varphi)$ im Vergleich mit $B_{\mathrm{M} 3 \mathrm{FM}}$ aus Widerstandsmessungen und Magnetisierungsmessungen bei $T \approx 1.4 \mathrm{~K}$ (siehe Abb.4.15. Speziell in der Orientierung $B \|[100]$ wird bereits bei Magnetfeldern um 3.0 T eine signalbestimmende Magnetisierungsrichtung parallel [110] beobachtet, im Gegensatz zum Phasendiagramm bei höherer Temperatur.

Die aus den Cantileverexperimenten folgende Hysterese der magnetischen Phasen kann nur für den Phasenübergang $\Delta B_{2}(\varphi)=B_{2}$ (Felderhöhung) $-B_{2}$ (Felderniedrigung) mit $\Delta B_{2}(\varphi) \leq \Delta B_{2}\left(\varphi=45^{\circ}\right) \leq 1.0 \mathrm{~T}$ angegeben werden. Für den Niederfeldübergang $B_{1}$ wird kein scharfer Übergang in den afm Grundzustand beobachtet. Das Meßsignal $\Delta C$ verschwindet für alle Orientierungen eher kontinuierlich bei Feldern $B_{1} \leq 0.3 \mathrm{~T}$. 


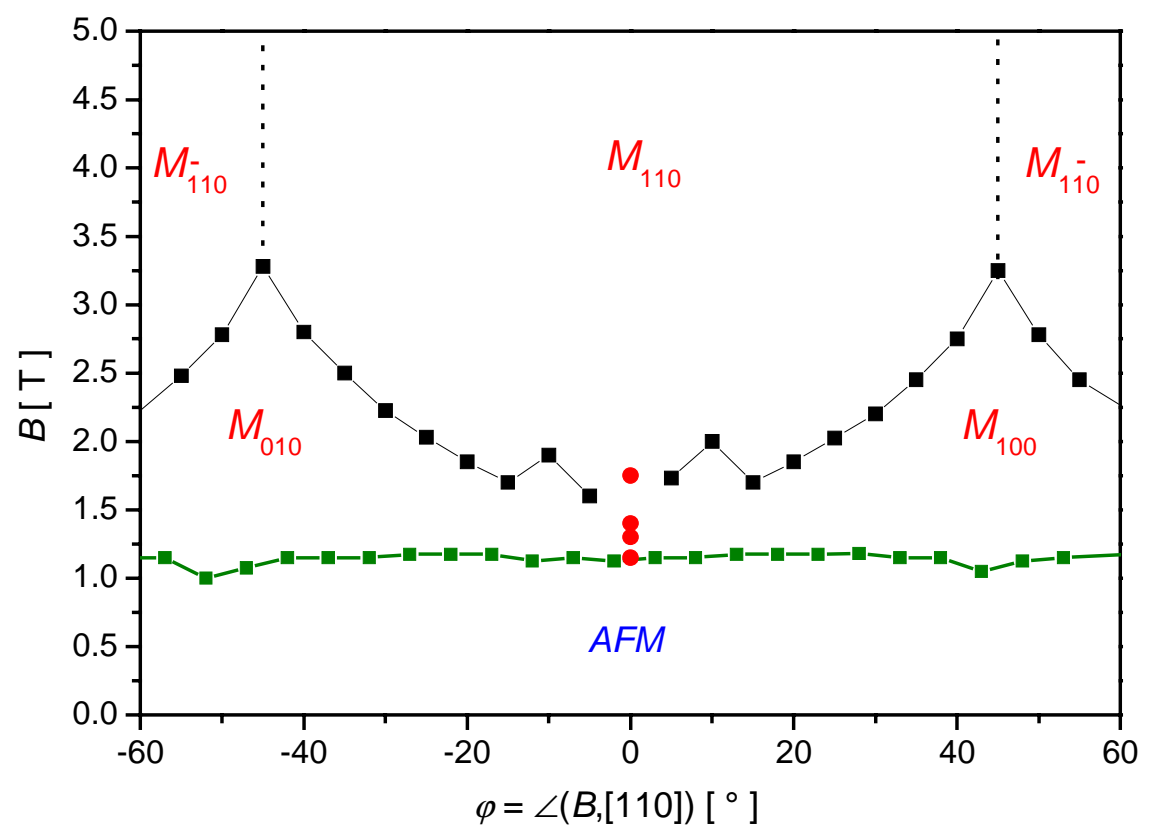

Abb.4.14 Phasendiagramm für $B \|(a, b)$-Ebene und $T=0.4 \mathrm{~K}$. Blau dargestellt ist die aus $B_{1>}(\varphi)$ ermittelte Phasengrenze und schwarz die entsprechende aus $B_{2>}(\varphi)$. Die Übergänge für $B \|$ [110] wurden der Messung für $B \|([110], c)$-Ebene entnommen.

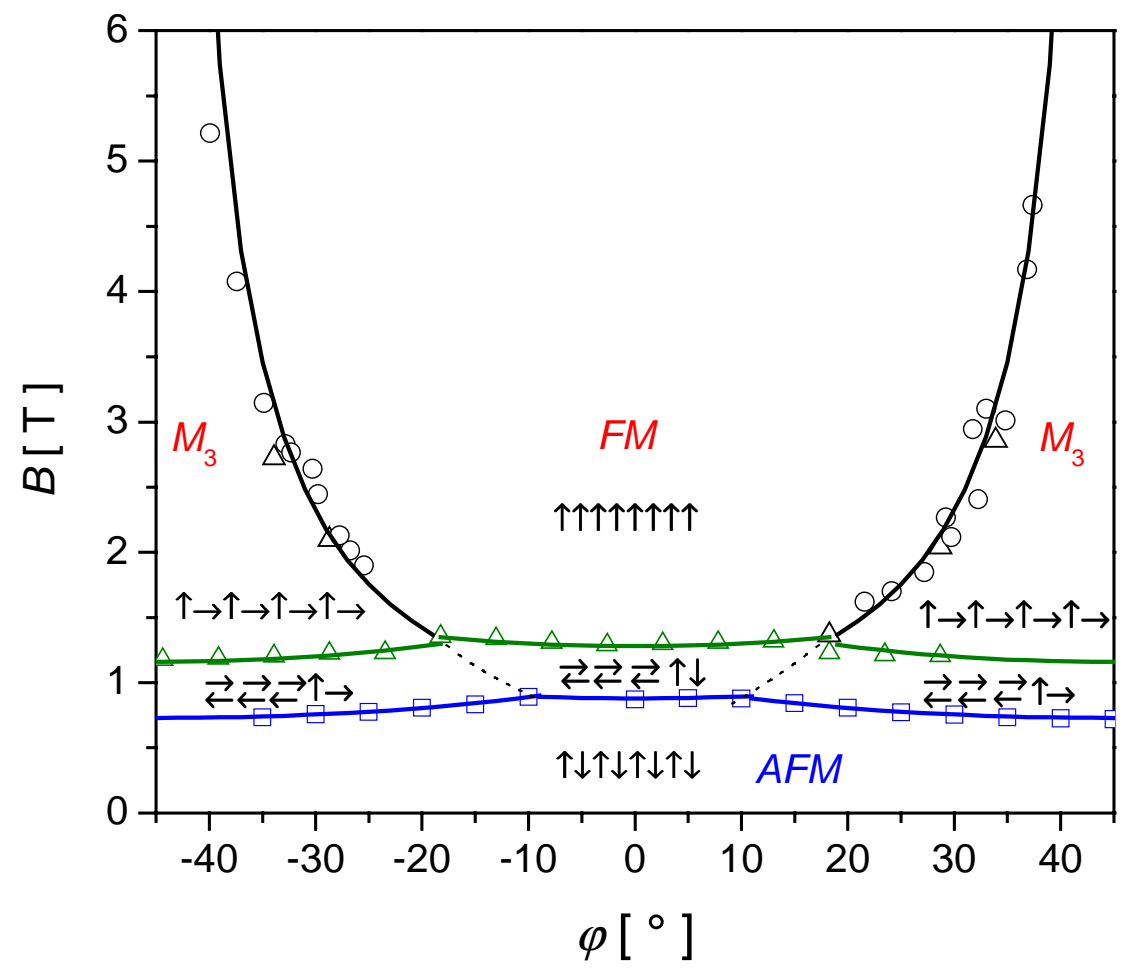

Abb.4.15 Phasendiagramm für $B \|(a, b)$-Ebene und $T=1.4 \mathrm{~K}$ nach [Pen99]. Die Pfeile ergeben die Struktur der magnetischen Phasen im angesprochenen ,4-clock“-Modell. 


\subsubsection{Neutronenstreuergebnisse}

\subsubsection{1 $B \|[110]$ und $T=2.0 \mathrm{~K}$}

Die Abb.4.16 zeigt die gemessenen Intensitäten $I(\vec{q}, \vec{B})$ dreier magnetischer BraggReflexe $\vec{q}_{1}=1 \vec{c}^{*} \equiv(001), \vec{q}_{2}=2 \vec{c}^{*} \equiv(002)$ und $\vec{q}_{2.75}=2.75 \vec{c}^{*} \equiv(002.75)$ als Funktion des langsam $(\mathrm{d} B / \mathrm{d} t \approx 1 \mathrm{~T} / \mathrm{h})$ ansteigenden Magnetfeldes $B \|[110]$ und konstanter Temperatur $T=2.0 \mathrm{~K}$. Die Intensität $I_{002.75}$ des Streuvektors $\vec{q}_{2.75}=2.75 \vec{c}^{*} \equiv(002.75)$ ist aus Darstellungsgründen mit 5-facher Überhöhung relativ zur tatsächlich gemessenen Intensität dargestellt.

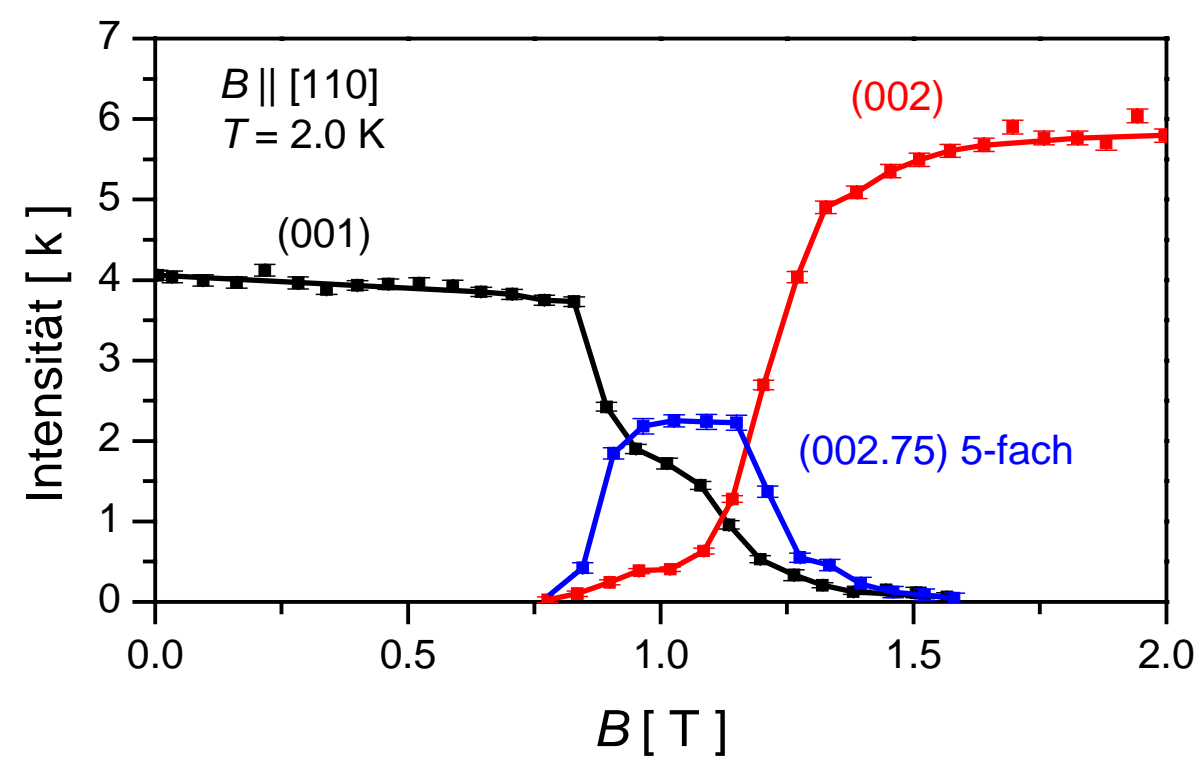

Abb.4.16 Intensität der magnetischen Reflexe (001), (002) und (002.75) in der Orientierung $B \|$ [110] für Felderhöhung $0 \mathrm{~T} \leq B \leq 2 \mathrm{~T}$.

Nach Kap.422 entspricht der Streuvektor $\vec{q}_{1}=1 \vec{c}^{*} \equiv(001)$ einer magnetischen Struktur mit Wellenvektor $\vec{k}_{1}=1 \vec{c}^{*}$ und Wellenlänge $\lambda_{1}=2 \pi /\left|\vec{c}^{*}\right|=1 \cdot c$ entlang (001) im Ortsraum. Aufgrund dieser Periodizitätslänge und dem Auftreten für Magnetfelder $B \leq 1.3 \mathrm{~T}$ kann $\vec{k}_{1}$ mit der aus anderen Neutronenstreuexperimenten im Nullfeld bekannten kommensurablen afm Struktur identifiziert werden (siehe Abb.1.2 nach [Lyn97]). Entsprechend ist dem Streuvektor $\vec{q}_{2}=2 \vec{c}^{*} \equiv(002)$ eine magnetische Struktur mit Wellenlänge 

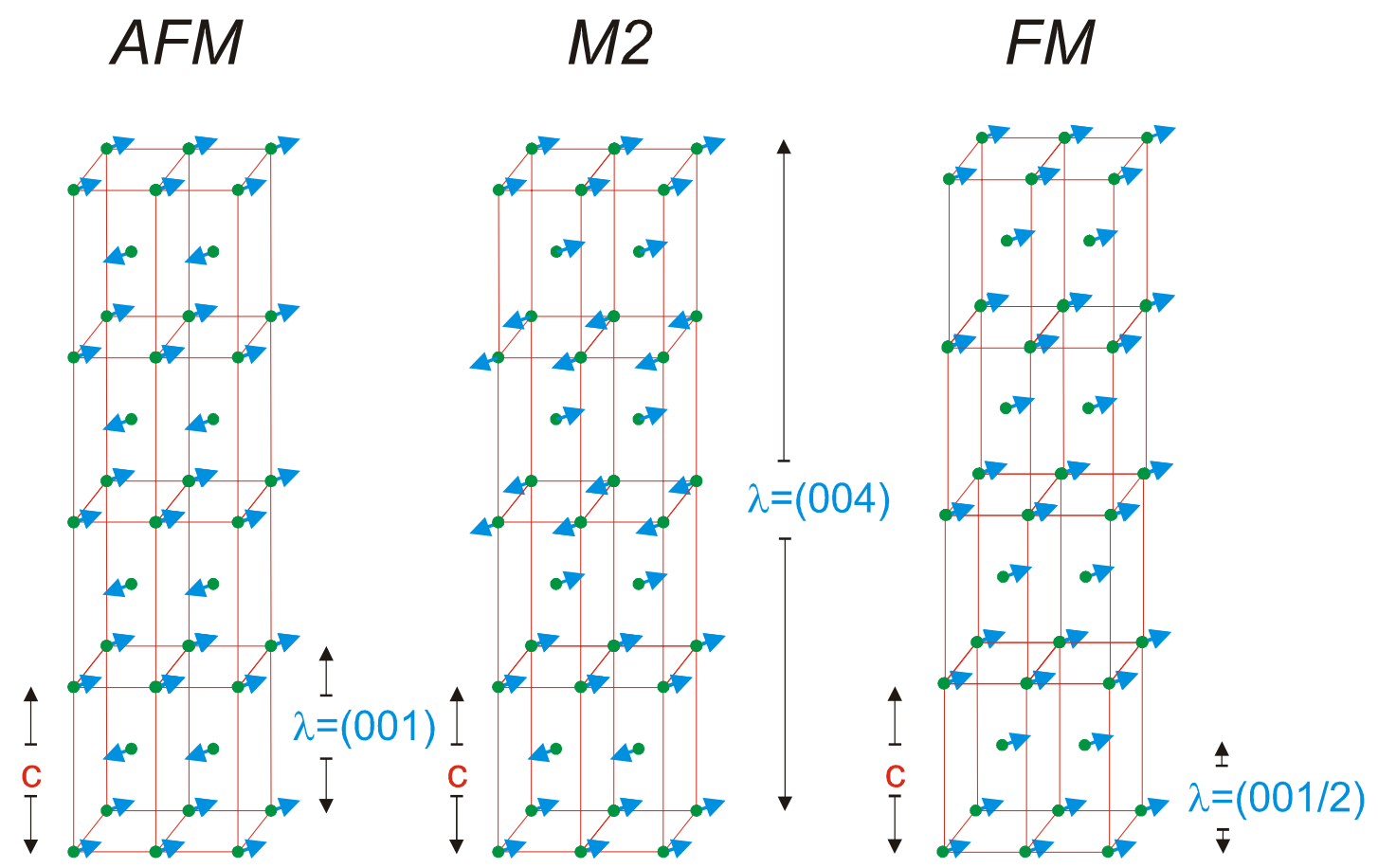

Abb.4.17 Schematische Darstellung dreier magnetischer Strukturen im ,"4-clock“-Model. Links die afm Struktur nach [Lyn97]. In der Mitte und rechts mögliche Realisierung der in DyNi ${ }_{2} B_{2} C$ auftretenden Zustände M2 und FM.

$\lambda_{2}=2 \pi /\left|2 \vec{c}^{*}\right|=(1 / 2) \cdot c$ zuzuordnen. Mit der Annahme eines vollständig gesättigten $\mathrm{Zu}-$ standes $F M$ oberhalb $B=1.3 \mathrm{~T}$ in der Orientierung $B \|[110]$ (siehe Abb.4.15) kann die Wellenlänge $\lambda_{2}$ mit einer vollständig gesättigten magnetischen Phase aus $f m$ ausgerichteten $(a, b)$-Ebenen mit Magnetisierung entlang [110] in Einklang gebracht werden (siehe Abb.4.17). Der Streuvektor $\vec{q}_{2.75}=2.75 \vec{c}^{*} \equiv(002.75)$ ist ein Satellitenreflex des wie (001) die afm Struktur repräsentierenden Streuvektors $\vec{q}_{3}=3 \vec{c}^{*} \equiv(003)$, d.h. er tritt symmetrisch auch bei $\vec{q}_{3.25}=3.25 \vec{c}^{*} \equiv(003.25)$ im Feldbereich $0.8 \mathrm{~T} \leq B \leq 1.3 \mathrm{~T}$ auf. Er entspricht damit einer dritten magnetischen Struktur mit Wellenvektor $\vec{k}_{0.25}=0.25 \vec{c}^{*}$ und Wellenlänge $\lambda_{4}=2 \pi /\left|0.25 \vec{c}^{*}\right|=4 \cdot c$ entlang (001) im Ortsraum. Dies könnte der in Abb.4.17 vorgeschlagenen Struktur mit der von Winzer et al. [Win99b] für diesen Feldbereich um $B=1.2 \mathrm{~T}$ berechneten Sättigungsmagnetisierung von $1 / 4 M_{\text {sat }}$ entsprechen. Bei 
zusätzlichen Experimenten über das hier gezeigte maximale Magnetfeld $B=2.0 \mathrm{~T}$ bis maximal $B=5.0 \mathrm{~T}$, wurden keine signifikanten Änderungen in den Intensitäten $I(\vec{q}, \vec{B})$ der Reflexe beobachtet [Kre99a]. Zur endgültigen Bestimmung der zu den magnetischen Reflexen gehörenden Strukturen über die hier genannten qualitativen Argumente hinaus sind weitere Messungen und Struktursimulationen in Planung [Kre00]. Hier werden Folgen von in den $(a, b)$-Ebenen entlang [110]-Richtungen $f m$ ausgerichteten magnetischen Momenten angenommen, was aus der Kenntnis des afm Grundzustandes geschlussfolgert wird [Lyn97]. Der Beweis für diese Einschränkung der Magnetisierungsrichtung innerhalb der Ebenen steht noch aus.

\subsubsection{2 $B \|[100]$ und $T=2.0 \mathrm{~K}$}

Die Abh.4.18 zeigt die gemessen Intensitäten $I(\vec{q}, \vec{B})$ der magnetischen Bragg-Reflexe $\vec{q}_{1}=1 \vec{c}^{*} \equiv(001), \vec{q}_{2}=2 \vec{c}^{*} \equiv(002)$ und $\vec{q}_{2.75}=2.75 \vec{c}^{*} \equiv(002.75)$ für langsam ansteigendes Magnetfeld $B \|[100]$ und $T=2.0 \mathrm{~K}$.

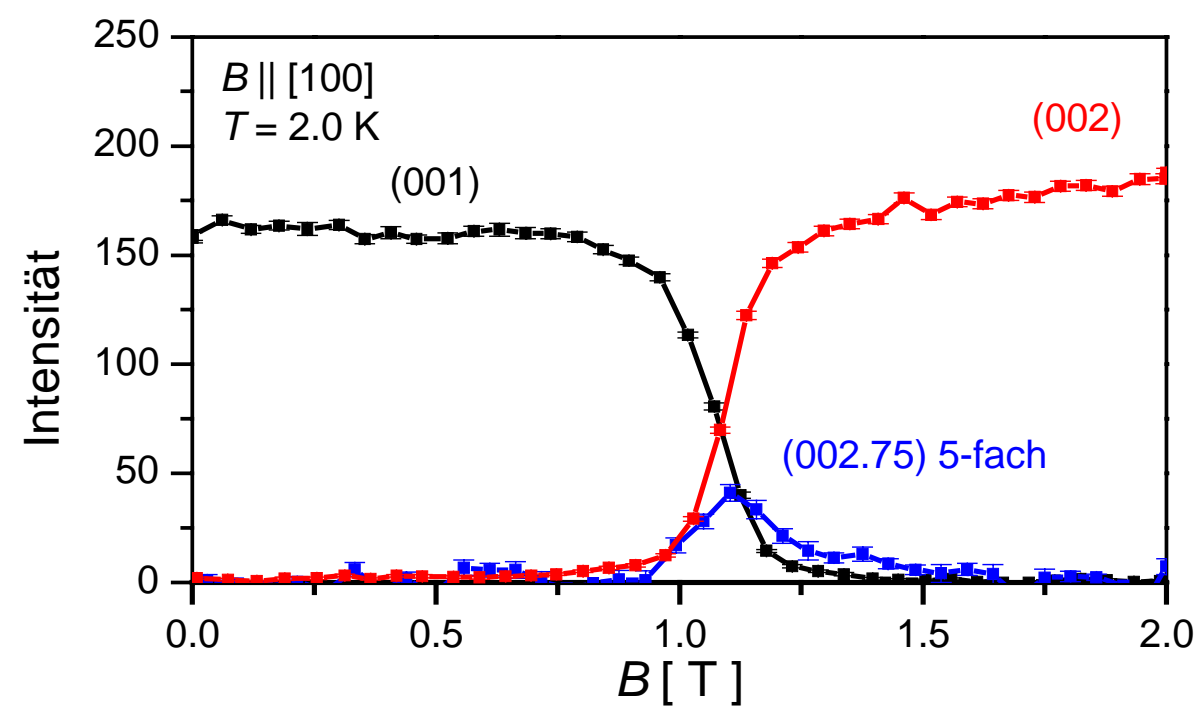

Abb.4.18 Intensität der magnetischen Reflexe (001), (002) und (002.75) in der Orientierung B || [100].

Die Feldabhängigkeiten $I(\vec{q}, \vec{B})$ sind im Magnetfeldbereich unter $B=2.0 \mathrm{~T}$ (siehe Abb.4.18 nahezu identisch zum Verhalten für $B \|[110]$. Oberhalb $B=2.0 \mathrm{~T}$ tritt in der Orientierung $B \|[100]$ allerdings ein zusätzlicher Anstieg in der Intensität des (002)Reflexes auf, der bei $B \approx 3.0 \mathrm{~T}$ etwa in der doppelten Intensität $I_{002}(3 \mathrm{~T}) \approx 2 \cdot I_{002}(2 \mathrm{~T})$ 


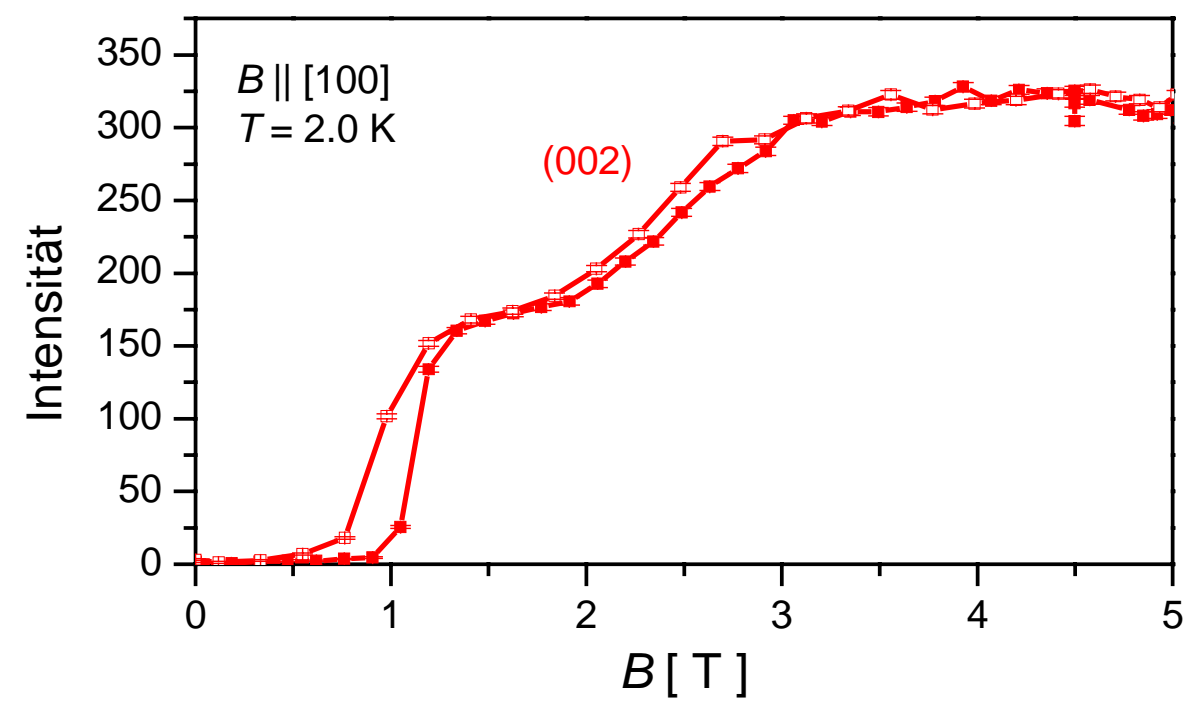

Abb.4.19 Intensität des Reflexes (002) bei Felderhöhung (geschlossene Symbole) und Felderniedrigung (offene Symbole) für $0 T \leq B \leq 5 \mathrm{~T}$.

vorliegt (siehe Abb.4.19). Eine mögliche Interpretation dieses Anstiegs ist der Zusammenschluß von Domänen. Wie für die Orientierung $B \|[110]$ beschrieben, repräsentiert der (002)-Streuvektor oberhalb etwa 1.2 T eine Struktur FM, aus in sich $f m$ ausgerichteten $(a, b)$-Ebenen mit Wellenlänge $\lambda_{2}=(1 / 2) \cdot c$ im Ortsraum (siehe Abb.4.17. Allerdings liegen für $B \|[100]$ zwei energetisch gleichberechtigte leichte $\langle 110\rangle$-Richtungen vor. So scheint bei $B=2.0 \mathrm{~T}$ eine Gleichverteilung von Domänen der Struktur FM mit Magnetisierungsrichtung in den Ebenen entlang [110] und von Domänen mit Magnetisierungsrichtung entlang $[1 \overline{1} 0]$ vorzuliegen. Der oberhalb $B=2.0 \mathrm{~T}$ beobachtete Anstieg in der Intensität $I_{002}$ entspricht einer Erhöhung des Ordungsgrades bei gleicher magnetischer Struktur, d.h. eine der Domänensorten wächst auf Kosten der anderen. Bei etwa $B=3.0 \mathrm{~T}$ ist eine Sättigungszustand erreicht, was aufgrund der verdoppelten Intensität $I_{002}(3 \mathrm{~T}) \approx 2 \cdot I_{002}(2 \mathrm{~T})$ als eindomäniger Zustand der Struktur $F M$ interpretiert werden kann. Mit dieser Interpretation des Verlaufs bei Felderhöhung muß aus dem gemessenen Intensitätsverlauf $I(002)$ aus Abb.4.19 bei Felderniedrigung geschlossen werden, daß bei $B \approx 2.0 \mathrm{~T}$ wieder ein zweidomäniger oder mehrdomäniger Zustand vorliegt. 


\subsection{Diskussion}

Die aus Cantileverexperimenten und Neutronenstreudaten gewonnenen Ergebnisse zeigen im Feldbereich $B \leq 1.2 \mathrm{~T}$ der magnetischen Zwischenphasen zum Teil gute Übereinstimmung mit den aus Vorarbeiten bekannten Beschreibungen [Pen99], [Can97a], [Nau98].

Die aus Cantilevermessungen ermittelte signalbestimmende Magnetisierungsrichtung ist für weite Teile des Winkelbereichs zwischen [110] und [100] mit der von Peng und Canfield bei $T \approx 2.0 \mathrm{~K}$ bestimmten im Einklang. Bei den hier für tiefere Temperaturen $T=0.4 \mathrm{~K}$ durchgeführten Cantilerexperimenten kann allerdings bis auf einen kleinen Winkelbereich um $B \|$ [110] nur auf Magnetisierungsrichtungen $M \|[100]$ geschlosssen werden, verglichen mit zwei verschiedenen Magnetisierungsrichtungen in $M 1$ und $M 2$ für $T=1.4 \mathrm{~K}$ (siehe Abb.4.15. Die für den von Peng beobachteten „reentrant"-Effekt entscheidende Hysterese der magnetischen Phasen kann nur für den Phasenübergang $B_{2}(\varphi)$ mit $\Delta B_{2}(\varphi) \leq \Delta B_{2}\left(\varphi=45^{\circ}\right) \leq 1.0$ T bestimmt werden.

Die Neutronenstreuexperimente ergeben erste Belege für die Ausbildung der feldinduzierten Zwischenphasen als periodische Strukturen entlang der [001]-Richtung, was bislang nur aus der Kenntnis der Struktur des afm Grundzustandes [Lyn97] und den im „4-clock“-Modell berechneten Strukturen gefolgert wurde. Eine mögliche zum Streuvektor $\vec{q}_{2.75}=2.75 \vec{c}^{*} \equiv(002.75)$ gehörende magnetische Struktur mit Wellenvektor $\vec{k}_{0.25}=0.25 \vec{c}^{*}$ kann im Einklang mit den bisherigen Annahmen über die magnetische Zwischenphase $M_{2}$ konstruiert werden.

Beide experimentellen Methoden weisen allerdings auf eine mikroskopisch andere Deutung der vorliegenden Phasen für Orientierungen $B$ nicht parallel zur leichten Richtung ( $B$ H [110]). Sowohl aus den Neutronenstreudaten als auch den Cantileverexperimenten scheint ein Mehrdomänenzustand für $B$ H [110] möglich. Die Existenz desselben folgt für die Cantileverergebnisse aus der Abweichung der dort beobachteten Winkelabhängigkeit $B_{2}(\varphi)$ verglichen mit der von Winzer et al. [Win99b] in einem Eindomänenmodell entwickelten Beschreibung. Im Fall von zusätzlichen feld- und phasenabhängigen Energiebeiträgen durch Domänenbildung und Domänenordnung können Abweichungen von der in Kap 4.1 ermittelten $1 / \cos (\varphi \pm x)$-Abhängigkeit der Phasengrenzen entstehen. Weiterhin kann das Ansteigen der Intensität des $q_{2}=2 \pi / c \cdot(002)$-Streuvektors in den Neutronen- 
streuexperimenten mit $B \|[100]$ als Domänenzusammenschluß interpretiert werden. Der Sättigungswert der Intensität wird dabei etwa beim gleichen Feldwert $B \approx 3.0 \mathrm{~T}$ wie $B_{2}\left(\varphi=45^{\circ}\right)=B_{2}(B \|[100])$ aus den Cantileverexperimenten erreicht.

Die Beobachtung des Streuvektors $\vec{q}_{2.75}=(002.75)$ für beide Orientierungen $B \|[110]$ und $B \|$ [100] könnte demnach zwar zur gleichen Struktur $M 2$ aus Abb.4.17 gehören, für $B \|[110]$ aber in einer Domäne mit Richtung der Dy-Momente entlang $\pm[110]$ und für $B$ || [100] in zwei Sorten von Domänen mit Richtung der Dy-Momente entlang $\pm[110]$ in der einen und entlang $\pm[1 \overline{1} 0]$ in der anderen.

Die hier gefundenen Indizien für Mehrdomäneneffekte scheinen hinsichtlich der Interpretation des Effekts der ,reentrant“ Supraleitung interessant. Die größere Paarbrechung in der Orientierung $B \|[100]$ gegenüber $B \|[110]$ nach einem Hysteresexperimente für Temperaturen $T \approx 50 \mathrm{mK}$ [Pen99] könnte damit nicht nur durch Anisotropien der magnetischen Zwischenphasen, sondern auch durch eine unterschiedliche felderzeugte Domänenstruktur bewirkt werden. 


\section{Zusammenfass ung}

In dieser Arbeit wurden die elektronischen und magnetischen Eigenschaften von einkristallinen SE-Borkarbiden mit verschiedene Meßmethoden charakterisiert.

Die Detektion von dHvA-Oszillationen im unmagnetischen und unterhalb $T_{\mathrm{c}}=16.3 \mathrm{~K}$ supraleitenden $\mathrm{LuNi}_{2} \mathrm{~B}_{2} \mathrm{C}$ ergab neue experimentelle Erkenntnisse über die vorliegenden Fermiflächen. So konnte für einen der beobachten Fermiflächenquerschnitte die Existenz einer teilweise verschwindenden Energielücke nachgewiesen werden.

Die detaillierte Charakterisierung der Winkelabhängigkeiten der untersuchten Fermiflächenquerschnitte ermöglichte die Identifikation für einen der beiden mit einer aus Bandstrukturrechnungen folgenden Elektronentasche. Aus den zugehörigen effektiven Elektronenmassen in den Querschnitten konnten zwei in $\mathrm{LuNi}_{2} \mathrm{~B}_{2} \mathrm{C}$ vorliegende Fermigeschwindigkeiten bestimmt werden.

Der Vergleich der hier gefunden Ergebnisse sowohl mit Aussagen aus vorliegenden Bandstrukturrechnungen als auch mit experimentellen Ergebnissen der verwandten Verbindung $\mathrm{YNi}_{2} \mathrm{~B}_{2} \mathrm{C}$ ergab Gemeinsamkeiten in der Geoemtrie der Fermiflächen und Unterschiede in den effektiven Massen, mit Tendenz zu größeren $m$ in $\mathrm{LuNi}_{2} \mathrm{~B}_{2} \mathrm{C}$ verglichen mit äquivalenten Querschnitten in $\mathrm{YNi}_{2} \mathrm{~B}_{2} \mathrm{C}$.

Durch die Charakterisierung der Winkelabhängigkeit in $B_{\mathrm{c} 2}(T, \alpha)$ bei tiefen Temperaturen konnten Vorhersagen zur $B_{\mathrm{c} 2}$-Anisotropie aus einem unkonventionellen $d$-Wellen-Modell zur Supraleitung experimentell widerlegt werden. Zusammen mit dem gemessenen $B_{\mathrm{c} 2}(T)$-Verlauf und den quantitativen Ergebnissen aus den dHvA-Experimenten wurden wichtige Grundlagen für die Beschreibung der Temperaturabhängigkeit des oberen kritischen Feldes in einem anisotropen Zwei-Band-Modell ermittelt, das die in vielen Borkarbiden beobachtete positive Krümmung in $B_{\mathrm{c} 2}(T)$ nahe $T_{\mathrm{c}}$ richtig beschreibt.

Bei der beobachteten Ähnlichkeit der Fermiflächen in $\mathrm{LuNi}_{2} \mathrm{~B}_{2} \mathrm{C}$ und $\mathrm{YNi}_{2} \mathrm{~B}_{2} \mathrm{C}$ sollten die dHvA-Experimente bei größeren Magnetfeldern $B>12 \mathrm{~T}$ fortgesetzt werden, um die in $\mathrm{YNi}_{2} \mathrm{~B}_{2} \mathrm{C}$ vorliegenden Querschnitte mit größerer effektiver Masse evtl. auch in $\mathrm{LuNi}_{2} \mathrm{~B}_{2} \mathrm{C}$ zu beobachteten. Weiterhin kann mit der Bestimmung von Anisotropien in den effektiven Massen eine Beschreibung des anisotropen $B_{\mathrm{c} 2}(\alpha, T)$-Verlaufes in der $(a, b)$ Ebene angestrebt werden. 
Durch Cantilever- und erste Neutronenstreuexperimente wurde die Kenntnis über die felderzeugten magnetischen Phasen im unterhalb $T_{\mathrm{N}}=10.3 \mathrm{~K}$ afm ordnenden und unterhalb $T_{\mathrm{c}}=6.8 \mathrm{~K}$ supraleitenden $\mathrm{DyNi}_{2} \mathrm{~B}_{2} \mathrm{C}$ erweitert. Mittels Cantileverexperimenten bei tiefster Temperatur wurde die Anisotropie der Magnetisierung innerhalb der $(a, b)$-Ebene des Systems untersucht, was zur Bestimmung von zwei magnetische Phasen und deren orientierungsabhängigen Grenzen führte.

Die Beobachtung des Streuvektors $\vec{q}_{2.75}=(002.75)$ in Neutronenstreuexperimenten bestätigt erstmals direkt das Auftreten von kommensurablen Stapelfolgen in den felderzeugten magnetischen Phasen in $\mathrm{DyNi}_{2} \mathrm{~B}_{2} \mathrm{C}$. Die beobachtete Anisotropie in der Feldabhängigkeit der Intensität $I_{002}$ des Streuvektors $\vec{q}_{2}=(002)$ aus den Neutronenstreuexperimenten und die Anisotropie der aus Cantileverexperimenten ermittelten Sättigungsphasen wurden als Mehrdomäneneffekte interpretiert. Weitere Neutronenstreuexperimente sind in Planung, um diese Interpretation zu untermauern.

Aufgrund des in anderen Experimenten beobachteten ähnlichen Verhaltens der magnetischen Borkarbide oberhalb $T=2.0 \mathrm{~K}$, sollten die hier bei tiefsten Temperaturen durchgeführten Cantileverexperimente an $\mathrm{DyNi}_{2} \mathrm{~B}_{2} \mathrm{C}$ auf andere $S E$-Borkarbide ausgedehnt werden. 


\section{Literaturverzei chnis}

[Abr61]: A. A. Abrikosov und L. P. Gorkov: Contribution to the theory of superconducting alloys with paramagnetic impurities. Sov. Phys. JETP 12, 1243-1253 (1961).

[Ami00]: A. Amici, P. Thalmeier und P. Fulde: Theoretical model for the superconducting and magnetically ordered borocarbides. Phys. Rev. Lett. 84, 1800-1803 (2000).

[Ami98]: A. Amici und P. Thalmeier: Microscopic theory of magnetic phase transitions in $\mathrm{HoNi}_{2} \mathrm{~B}_{2} \mathrm{C}$. Phys. Rev. B 57, 10684-10687 (1998).

[Ash76]: N. W. Ashcroft und N. D. Mermin: Solid state physics. W. B. Saunders, Philadelphia (1976).

[Bar57]: J. Bardeen, L. N. Cooper und J. R. Schrieffer: Theory of superconductivity. Phys. Rev. 108, 1175-1204 (1957).

[Bed86]: J. G. Bednorz und K. A. Müller: Possible high $T_{c}$ superconductivity in a Ba-LaCu-O system. Z. Phys. B 64, 189-193 (1986).

[Bed87]: J. G. Bednorz, M. Takashige und K. A. Müller: Susceptibility measurements support high $T_{C}$ superconductivity in the Ba-La-Cu-O system. Europhys. Lett. 3, 379-385 (1987).

[Bit97]: H. Bitterlich: Magnetische Anisotropie von $\mathrm{TbNi}_{2} \mathrm{~B}_{2} \mathrm{C}$. Diplomarbeit: Georg August Universität, Göttingen (1997)

[Bra88]: E. H. Brandt: Magnetic field density of perfect and imperfect flux line lattices in type II superconductors. I. Application of periodic solutions. J. Low Temp. Phys 73, 355390 (1988).

[Buc93]: W. Buckel: Supraleitung. VCH, Weinheim (1993).

[Bud95]: S. L. Budko et al. : Effect of the Ni site substitution on superconducting properties of $Y \mathrm{Ni}_{2} \mathrm{~B}_{2} \mathrm{C}$. Physica C 243, 183-186 (1995).

[Bur96]: P. Burlet: D15-Homepage. http://www. ill. fr/YellowBook/D15/ (1996)

[Cam95]: A. v. Campenhausen: Untersuchungen der oberen kritischen Feldstärke an unkonventionellen Supraleitern. Diplomarbeit: Georg August Universität, Göttingen (1995)

[Can96]: P. C. Canfield, S. L. Budko und B. K. Cho: Possible coexistence of superconductivity and weak ferromagnetism in $\mathrm{ErNi}_{2} \mathrm{~B}_{2} \mathrm{C}$. Physica C 262, 249-254 (1996). 
[Can97]: P. C. Canfield et al. : Angular dependence of metamagnetic transitions in Ho$\mathrm{Ni}_{2} \mathrm{~B}_{2} \mathrm{C}$. Phys. Rev. B 55, 970-976 (1997).

[Can97a]: P. C. Canfield und S. L. Budko: Angular dependence of metamagnetic transitions in $R \mathrm{Ni}_{2} \mathrm{~B}_{2} \mathrm{C}(\mathrm{R}=\mathrm{ER}, \mathrm{Ho}, \mathrm{Dy}$ and $\mathrm{Tb})$. JALCOM 262-263, 169-174 (1997).

[Can98]: P. C. Canfield, P. L. Gammel und D. J. Bishop: New magnetic superconductors: A toy box for solid state physicists. Phys. Today 51, 40-46 (1998).

[Car94]: S. A. Carter et al. : Electron density of states in the borocarbide intermetallic superconductors. Phys. Rev. B 50, 4216-4219 (1994).

[Cav94]: R. J. Cava et al. : Superconductivity at $23 \mathrm{~K}$ in yttrium palladium boride carbide. Nature 367, 146-148 (1994).

[Cho95a]: B. K. Cho et al. : Magnetism and superconductivity in single-crystal Er$\mathrm{Ni}_{2} \mathrm{~B}_{2} \mathrm{C}$. Phys. Rev. B 52, 3684-3695 (1995).

[Cho96]: B. K. Cho et al. : Crystalline electric-field effects in single-crystal $\mathrm{HoNi}_{2} \mathrm{~B}_{2} \mathrm{C}$. Phys. Rev. B 53, 2217-2220 (1996).

[Din52]: R. B. Dingle: Some magnetic properties of metals II. The influence of collisions on the magnetic behaviour of large systems. Proc. Roy. Soc. A 211, 517-525 (1952).

[Dre99]: S. -L. Drechsler et al. : Superconducting rare earth transition metal borocarbides. Physica C 317-318, 117-126 (1999).

[Eli60]: G. M. Eliashberg: Interactions between electrons and lattice vibrations in a superconductor. Sov. Phys. JETP 11, 696-702 (1960).

[Esk97]: M. R. Eskildsen et al. : Structural stability of the square flux line lattice in $\mathrm{YNi}_{2} \mathrm{~B}_{2} \mathrm{C}$ and $\mathrm{LuNi}{ }_{2} \mathrm{~B}_{2} \mathrm{C}$ studied with small angle neutron scattering. Phys. Rev. Lett. 79, 487-490 (1997).

[Fü198]: K. Fülber et al. : Magnetostriction due to flux pinning in polycrystalline $\mathrm{Ni}_{2} \mathrm{~B}_{2} \mathrm{C}$. Physica C 299, 1-8 (1998).

[Fur95]: A. Furrer: Magnetic Neutron Scattering. Proceedings of the third summer school on neutron scattering, Zuoz (1995)

[Gey87]: U. Geyer: Aufbau eines ${ }^{3}$ He-Hochfeldkryostaten für Untersuchungen des de Haas-van Alphen Effekts mit einer niederfrequenten Feldmodulationsmethode. Diplomarbeit: Georg August Universität, Göttingen (1987) 
[Gol94]: A. I. Goldman et al. : Magnetic pair breaking in $\mathrm{HoNi}_{2} \mathrm{~B}_{2} \mathrm{C}$. Phys. Rev. B 50, 9668-9671 (1994).

[Gom97]: F. Gompf et al. : Lattice vibrations and electron-phonon coupling in superconducting quarternary borocarbides: An inelastic neutron scattering investigation. Physical Review B 55, 9058-9066 (1997).

[Gra76]: J. E. Graebner und M. Robbins: Fermi surface measurements in normal and superconducting $2 \mathrm{H}-\mathrm{NbSe}_{2}$. Phys. Rev. Lett. 36, $422-425$ (1976).

[Gsc82]: E. Gratz und M. J. Zuckermann: Transport properties (electrical resistivity, thermoelectric power and thermal conductivity) of rare earth intermetallic compounds in K. A. GschneiderJr. und L. R. Eyring: Handbook on the physics and chemistry of rare earths. 5 North-Holland, Amsterdam (1982), 117ff

[Hei95]: M. Heinecke und K. Winzer: de Haas-van Alphen effect in the superconducting state of $\mathrm{YNi}_{2}{ }_{2} C$. Z. Phys. B 98, 147-150 (1995).

[Hei95]: M. Heinecke: Messungen des de Haas-van Alphen Effekts an den Supraleitern $\kappa-E T_{2} I_{3}$ und $Y N i_{2} B_{2} C$. Dissertation: Georg August Universität, Göttingen (1995)

[Hil99]: G. Hilscher und H. Michor: Superconductivity and Magnetism in Quaternary Borocarbides and Bornitrides in A. V. Narlikar: Studies of High Temperature Superconductors. 28 Nova Science Publishers, New York (1999), 241ff

[Hon94]: N. M. Hong et al. : Superconductivity in Y-Ni-B base compounds. Physica C 227, 85-94 (1994).

[Jan98]: T. J. Janssen et al. : Quantitative investigation of the de Haas-van Alphen effect in the superconducting state. Phys. Rev. B 57, 11698-11715 (1998).

[Kal98]: V. A. Kalatsky und V. L. Pokrovsky: Microscopic model for the magnetic subsystem in $\mathrm{HoNi}_{2} \mathrm{~B}_{2} \mathrm{C}$. Phys. Rev. B 57, 5485-5488 (1998).

[Kim95]: H. Kim, C. D. Hwang und J. Ihm: ab initio pseudopotential calculations for the electronic structure of low- $T_{c} \mathrm{LuNi}_{2} \mathrm{~B}_{2} \mathrm{C}$ and the related compound LuNiBC. Phys. Rev. B 52, 4592-4596 (1995).

[Kit88]: C. Kittel: Einführung in die Festkörperphysik. Oldenbourg-Verlag, München (1988).

[Kno96]: D. Knoll: Magnetfeldabhängige Messungen der spezifischen Wärme von $\mathrm{TmNi}_{2} \mathrm{~B}_{2} \mathrm{C}$. Diplomarbeit: Georg August Universität, Göttingen (1996) 
[Kre00]: A. Kreyssig: in Vorbereitung. Dissertation: Institut für Angewandte Physik, Dresden (2000)

[Kre99]: A. Kreyssig et al. : Evidence of tetragonal to orthorhombic distortion of Ho$\mathrm{Ni}_{2} \mathrm{~B}_{2} \mathrm{C}$ in the magnetically ordered state. J. Appl. Phys. 85, 6058-6060 (1999).

[Kre99a]: A. Kreyssig: Persönliche Mitteilung, (1999)

[Kru96]: K. Krug, M. Heinecke und K. Winzer: Upper-critical field anisotropy and magnetic phase diagram of $\mathrm{HoNi}_{2} \mathrm{~B}_{2}$ C. Physica C 267, 321-329 (1996).

[Kru96]: K. Krug: Anisotropien in den supraleitenden und magnetischen Eigenschaften von $\mathrm{HoNi}_{2} \mathrm{~B}_{2} C$. Diplomarbeit: Georg August Universität, Göttingen (1996)

[Ku94]: H. C. Ku et al. : Superconductivity at $15 \mathrm{~K}$ in the metastable $\mathrm{ScNi}{ }_{2} \mathrm{~B}_{2} \mathrm{C}$ compound. Phys. Rev. B 50, 351-353 (1994).

[Lan30]: L. Landau: Diamagnetismus der Metalle. Z. Phys. 64, 629-637 (1930).

[Lar79]: A. I. Larkin und Y. V. Ovchinnikov: Pinning in Type II Superconductors. J. Low Temp. Phys. 34, 409-428 (1979).

[Lif56]: I. M. Lifshitz und A. M. Kosevich: Theory of magnetic susceptibility in metals at low temperatures. Sov. Phys. JETP 2, 636-645 (1956).

[Lyn97]: J. W. Lynn et al. : Magnetic order and crystal structure in the superconducting $\mathrm{RNi}_{2} \mathrm{~B}_{2} \mathrm{C}$ materials. Phys. Rev. B 55, 6584-6598 (1997).

[Mak96]: K. Maki und H. Won: Why d-wave superconductivity? J. de Physique I France 6, 2317-2326 (1996).

[Man00]: S. Manalo et al. : Superconducting properties of $Y_{x} L u_{1-x} N i_{2} B_{2} C$ and $\mathrm{La}_{3} \mathrm{Ni}_{2} \mathrm{~B}_{2} \mathrm{~N}_{3-\delta}$ Acomparism between experiment and Eliashberg theory. eingereicht bei Phys. Rev. B (2000).

[Mas95]: M. ElMassalami et al. : The interplay between magnetism and superconductivity in $R N i_{2} B_{2} C(R=L u, T m, E r, H o, D y, T b, G d)$. Physica C 244, 41-48 (1995).

[Mat58]: B. T. Matthias, H. Suhl und E. Corenzwit: Spin exchange in superconductors. Phys. Rev. Lett. 1, 92-94 (1958).

[Mat84]: T. Matsubra: Superconductivity in Magnetic and Exotic Materials. Springer Verlag, Berlin (1984). 
[Mat94]: L. F. Mattheiss: Electronic properties of superconducting $\mathrm{LuNi}_{2} \mathrm{~B}_{2} \mathrm{C}$ and related boride carbide phases. Phys. Rev. B 49, 13279-13282 (1994).

[Mat94a]: L. F. Mattheiss, T. Siegrist und R. J. Cava: Superconductivity in the $\mathrm{LnNi}_{2} \mathrm{~B}_{2} \mathrm{C}$ intermetallics via Boron $A_{1 g}$ Phonons. Sol. State Comm. 91, 587-590 (1994).

[McP98]: D. McPaul et al. : Nonlocal effects and vortex lattice in $Y \mathrm{Ni}_{2} \mathrm{~B}_{2} \mathrm{C}$. Phys. Rev. Lett. 80, 1517-1520 (1998).

[Met97]: V. Metlushko et al. : Anisotropic upper critical field of $\mathrm{LuNi}_{2} \mathrm{~B}_{2} \mathrm{C}$. Phys. Rev. Lett 79, 1738-1741 (1997).

[Mod96]: R. Modler et al. : First-order transition between weak and strong pinning in clean superconductors with enhaced spin susceptibility. Phys. Rev. Lett. 76, 1292-1295 (1996).

[Mun96]: M. O. Mun et al. : Vortex glass and lattice melting transitions in a $\mathrm{YNi}_{2} \mathrm{~B}_{2} \mathrm{C}$ single crystal. Phys. Rev. Lett. 76, 2790-2793 (1996).

[Nag94]: R. Nagarajan et al. : Bulk superconductivity at an elevated temperature $\left(T_{c}\right.$ approximately $=12 \mathrm{~K}$ ) in a nickel containing alloy system $\mathrm{Y}$-Ni-B-C. Phys. Rev. Lett. 72, 274-277 (1994).

[Nau96]: D. G. Naugle et al. : Transport and magnetic measurements on single crystal rare earth-nickel-borocarbides. Czech. J. Phys. 46, 3263-3270 (1996).

[Nau98]: D. G. Naugle: Metamagnetic phases and interplay with superconductivity of single crystal $\mathrm{DyNi}_{2} \mathrm{~B}_{2}$ C. J. Mod. Phys. 12, 3174-3178 (1998).

[Ngu96]: L. H. Nguyen et al. : Fermi surfacestudies of the borocarbide superconductor $Y \mathrm{Ni}_{2} \mathrm{~B}_{2} C$. J. Low Temp. Phys. 105, 1653-1658 (1996).

[Noh97]: M. Nohara et al. : Magnetic field dependence of the low-temperature specific heat of the borocarbide superconductor $\mathrm{LuNi}_{2} B_{2} C$. J. Phys. Soc. Jap. 66, 1888-1891 (1997).

[Ove95]: G. Overbeck: Aufbau eines Mischungskryostaten und Messung der spezifischen Wärme von $\mathrm{YbBa}_{2} \mathrm{Cu}_{3} \mathrm{O}_{7}$ und $\mathrm{TmNi}_{2} \mathrm{~B}_{2} \mathrm{C}$. Diplomarbeit: Georg August Universität, Göttingen (1995)

[Par69]: R. D. Parks: Superconductivity. , New York (1969).

[Pen00]: Z. Q. Peng, K. Krug und K. Winzer: Supraleitung im zwischenvalenten Ce$\mathrm{Ni}_{2} \mathrm{~B}_{2} \mathrm{C}$. DPG-Frühjahrstagung, Regensburg (2000) 
[Pen98]: Z. Q. Peng, K. Krug und K. Winzer: Large hysteresis effect and reentrant behavior in $\mathrm{DyNi}_{2} \mathrm{~B}_{2} \mathrm{C}$ at temperatures $T<2 \mathrm{~K}$. Phys. Rev. B 57, R8123-8126 (1998).

[Pen99]: Z. Q. Peng: Superconductivity, magnetism and their interplay in quaternary $R \mathrm{Ni}_{2} \mathrm{~B}_{2} \mathrm{C}$ compounds. Dissertation: Georg August Universität, Göttingen (1999)

[Pen99a]: Z. Q. Peng: Persönliche Mitteilung, (1999)

[Pic94]: W. E. Pickett und D. J. Singh: $\mathrm{LuNi}_{2} \mathrm{~B}_{2} \mathrm{C}:$ A novel Ni - based strong coupling superconductor. Phys. Rev. Lett. 72, 3702-3705 (1994).

[Pin99]: M. Pinkpank et al. : The effect of oxygen reduction on the magnetic properties of $\mathrm{REBa}_{2} \mathrm{Cu}_{3} \mathrm{O}_{6+x}$. Physica C 317-318, 299-303 (1999).

[Rat96]: K. D. D. Rathnayaka et al. : Anisotropic magnetoresistance of single-crystal $\mathrm{HoNi}_{2} \mathrm{~B}_{2} \mathrm{C}$ and the interplay of magnetic and superconducting order. Phys. Rev. B 53, 5688-5695 (1996).

[Rat97]: K. D. D. Rathnayaka et al. : Transport and superconducting properties of $R \mathrm{Ni}_{2} \mathrm{~B}_{2} C(R=Y$, Lu) single crystals. Phys. Rev. B 55, 8506-8519 (1997).

[Rhe95]: J. Y. Rhee, X. Wang und B. N. Harmon: Generalized susceptibility and magnetic ordering in rare-earth nickel boride carbides. Phys. Rev. B 51, 15585-15587 (1995).

[Ros00]: H. Rosner et al. : Superconducting rare earth transition metal borocarbides. DPG-Frühjahrstagung, Regensburg (2000)

[Ros00]: H. Rosner: Persönliche Mitteilung, (2000)

[Sch94]: H. Schmidt, M. Müller und H. F. Braun: Superconductivity in the pseudoquarternary system $Y\left(\mathrm{Ni}_{1-x} \mathrm{Co}_{x}\right)_{2} \mathrm{~B}_{2} \mathrm{C}$. Physica C 235-240, 779-780 (1994).

[Sho84]: D. Shoenberg: Magnetic oscillations in metals. University Press, Cambridge (1984).

[Shu98]: S. V. Shulga et al. : Upper critical field peculiarities of superconducting $\mathrm{YNi}_{2} \mathrm{~B}_{2} \mathrm{C}$ and $\mathrm{LuNi}_{2} \mathrm{~B}_{2} \mathrm{C}$. Phys. Rev. Lett. 80, 1730-1733 (1998).

[Sie94]: T. Siegrist et al. : The crystal structure of superconducting $\mathrm{LuNi}_{2} \mathrm{~B}_{2} \mathrm{C}$ and the related phase LuNiBC. Nature 367, 254-256 (1994).

[Sin89]: K. P. Sinha und S. L. Kakani: Magnetic Superconductors: Recent Developments. Nova Science, New York (1989). 
[Sin95a]: D. J. Singh: Persönliche Mitteilung, (1995)

[Sin96]: D. J. Singh: Electronic properties of $\mathrm{YNi}_{2} \mathrm{~B}_{2}$ C. Sol. State Comm. 98, 899-902 (1996).

[Thi92]: N. Thier-Weigang: Vergleich von Temperaturmodulationsmethode und Feldmodulationsmethode zur Messung des de Haas-van Alphen Effekts. Dissertation: Georg August Universität, Göttingen (1992)

[Tim51]: S. Timoshenko und J. N. Goodier: Theory of elasticity. McGraw Hill, New York (1951).

[Tok95]: M. Tokunaga et al. : de Haas van Alphen oscillations of LuNi ${ }_{2} B_{2} C$ in pulsed high magnetic fields. J. Phys. Soc. Jap. 64, 1458-1461 (1995).

[Wan98]: G. Wang und K. Maki: Possible d-wave superconductivity in borocarbides: Upper critical field of $\mathrm{YNi}_{2} \mathrm{~B}_{2} \mathrm{C}$ and $\mathrm{LuNi}_{2} \mathrm{~B}_{2} \mathrm{C}$. Phys. Rev. B 58, 6493-6496 (1998).

[Wi197]: Y. deWilde et al. : Scanning tunneling microscopy observation of a square Abrikosov lattice in $\mathrm{LuNi}_{2} \mathrm{~B}_{2} \mathrm{C}$. Phys. Rev. Lett. 78, 4273-4276 (1997).

[Win99b]: K. Winzer, Z. Q. Peng und K. Krug: Superconductivity and the magnetic phase diagram of $\mathrm{DyNi}{ }_{2} \mathrm{~B}_{2} \mathrm{C}$ at very low temperatures. Physica B 259-261, 586-587 (1999).

[Zim75]: J. M. Ziman: Prinzipien der Festkörpertheorie. Verlag Harry Deutsch, Frankfurt a. M. (1975). 


\section{Danksagung}

Prof. Dr. Klaus Winzer danke ich für die interessante Aufgabenstellung und die engagierte Betreuung. Sein Interesse am Fortgang der vorliegenden Arbeit und die stete Diskussionsbereitschaft haben mich immer sehr motiviert.

Ein besonderer Dank gilt Prof. Dr. Gunther von Minnigerode ( $†$ Okt. 1998), der mir stets Vorbild und wohlwollender Ratgeber war.

Ich danke Dr. Zhiqiang Peng für viele anregende Diskussionen über die in den Borkarbiden auftretenden Phänomene. Er war mir ein netter und hifsbereiter Kollege.

Dr. Martin Heinecke danke ich für die Einarbeitung in die Kunst des dHvA-Effektes und seine Diskussionsbereitschaft über Fragestellungen jeder Art. Seine Begeisterung für die Physik war mit ein Grund für eine angenehme Arbeitsatmosphäre.

Die Zusammenarbeit mit Prof. S.-L. Drechsler und H. Rosner habe ich als produktives Zusammenspiel von theoretischer und experimenteller Physik empfunden.

Ich danke Andreas Kreyssig für die freundschaftliche Zusammenarbeit bei den Neutronenstreuexperimenten und die fruchtbaren Diskussionen nicht nur während der Experimente. Diese ließen uns auch manchen Meßmarathon unbeschadet überstehen.

Ich danke allen technischen Mitarbeitern des I. Physikalischen Institutes für die prompte und freundliche Hilfe bei allen anfallenden Arbeiten. Insbesondere sei Hartmut Eichenberg und Carsten Mahn gedankt.

Ich habe die gute Arbeitsatmosphäre im I.Physikalischen Institut immer sehr genossen. Dazu haben die Tennisrunde, Henning Harms, Jens Hoffmeister, Frank Leuenberger, Dr. Wiebke Lohstroh, Markus Münzenberg, Matthias Bicker, Prof. Dr. Wolfgang Felsch, PD Dr. Uli Geyer, Dr. Susanne Schneider, die „Crew Augsburg“ mit Prof. Dr. Konrad Samwer und viele weitere auch ehemalige Kollegen und Kolleginnen beigetragen. Ihnen allen sei dafür herzlichst gedankt.

Mein spezieller Dank richtet sich an Dr. Ruth Lüke, die mich über alle Höhen und Tiefen meines Werdegangs in der Physik begleitet und unterstützt hat.

Ich danke meinen Eltern und meiner Familie für die Unterstützung und Anteilnahme an meiner Arbeit.

Nicht vergessen seien die Jungs der ersten Stunde Stephan, Peter, Patrick und McFly. 


\section{Lebenslauf}

Persönliche Daten:

Name:

Klaus Krug

Geburtstag:

02.04.1971

Geburtsort:

Kassel

\section{Schulausbildung:}

1977-1981

1981-1990

Grundschule in Edermünde-Besse

1990

Gymnasium König-Heinrich-Schule in Fritzlar

Allg. Hochschulreife

\section{Wehrdienst:}

1990-1991

Wehrdienst in Hofgeismar und Fritzlar

\section{Hochschulausbildung:}

1991-1993

1993

1993-1996

1995-1996

1996

$1997-2000$
Physikstudium an der TU Darmstadt

Diplomvorprüfung im Fach Physik an der TU Darmstadt

Physikstudium an der Georg-August-Universität zu Göttingen

Diplomarbeit bei Prof. Dr. K. Winzer, I. Physikalisches Institut der Universität Göttingen;

Thema: Anisotropien in den supraleitenden und magnetischen Eigenschaften von $\mathrm{HoNi}_{2} \mathrm{~B}_{2} \mathrm{C}$

Diplomprüfung in Physik

Wissenschaftlicher Mitarbeiter am I. Physikalischen Institut der Universität Göttingen;

Anfertigung der vorliegenden Dissertation bei

Prof. Dr. K. Winzer 